\title{
Blood-contacting biomaterials for critical clinical applications
}

Citation for published version (APA):

Stevens, K. N. J. (2011). Blood-contacting biomaterials for critical clinical applications. [Doctoral Thesis, Maastricht University]. Maastricht University. https://doi.org/10.26481/dis.20111215ks

Document status and date:

Published: 01/01/2011

DOI:

$10.26481 /$ dis.20111215ks

Document Version:

Publisher's PDF, also known as Version of record

\section{Please check the document version of this publication:}

- A submitted manuscript is the version of the article upon submission and before peer-review. There can be important differences between the submitted version and the official published version of record.

People interested in the research are advised to contact the author for the final version of the publication, or visit the DOI to the publisher's website.

- The final author version and the galley proof are versions of the publication after peer review.

- The final published version features the final layout of the paper including the volume, issue and page numbers.

Link to publication

\footnotetext{
General rights rights.

- You may freely distribute the URL identifying the publication in the public portal. please follow below link for the End User Agreement:

www.umlib.nl/taverne-license

Take down policy

If you believe that this document breaches copyright please contact us at:

repository@maastrichtuniversity.nl

providing details and we will investigate your claim.
}

Copyright and moral rights for the publications made accessible in the public portal are retained by the authors and/or other copyright owners and it is a condition of accessing publications that users recognise and abide by the legal requirements associated with these

- Users may download and print one copy of any publication from the public portal for the purpose of private study or research.

- You may not further distribute the material or use it for any profit-making activity or commercial gain

If the publication is distributed under the terms of Article $25 \mathrm{fa}$ of the Dutch Copyright Act, indicated by the "Taverne" license above, 


\section{Blood-contacting biomaterials}

for critical clinical applications 
(C) Copyright K.N.J. Stevens, Maastricht 2011

ISBN 9789090264806

Printed by Drukkerij Duplica

Cover: "Sangre de plata" by Jean-Michel Perrillat, with kind permission. 


\title{
Blood-contacting biomaterials for critical clinical applications
}

\author{
PROEFSCHRIFT \\ ter verkrijging van de graad van doctor \\ aan de Universiteit Maastricht, \\ op gezag van de Rector Magnificus, \\ Prof. mr. G.P.M.F. Mols, \\ volgens het besluit van het College van Decanen, \\ in het openbaar te verdedigen op \\ donderdag 15 december 2011 om 10.00 uur
}

door

Kris Nico Jean Stevens 


\section{Promotores:}

Prof. dr. J.G. Maessen

Prof. dr. ir. L.H. Koole

\section{Beoordelingscommissie:}

Prof. dr. H.C. Schouten (voorzitter)

Prof. dr. J. Feijen (Universiteit Twente)

Prof. dr. D. Klee (Rheinisch-Westfaelische Technische Hochschule Aachen)

Prof. dr. L.W. van Rhijn

The author appreciates financial support for his dissertation.

Financial support was given by DSM Biomedical, Metalor Technologies SA, Vygon NL BV and MC Tec BV.

Financial support by the Dutch Heart Foundation for the publication of this thesis is gratefully acknowledged. 


\section{Table of contents}

Chapter 1 General introduction

Chapter 2 Bioengineering of improved biomaterials coatings for extracorporeal circulation requires extended observation of blood-biomaterial interaction under flow

Chapter 3 The relationship between the antimicrobial effect of catheter coatings containing silver nanoparticles and the coagulation of contacting blood

Chapter 4 Disruption and activation of blood platelets in contact with an antimicrobial composite coating consisting of a pyridinium polymer and silverbromide $(\mathrm{AgBr})$ nanoparticles

Chapter 5 Hydrophilic surface coatings with embedded biocidal silver nanoparticles and sodium heparin for central venous catheters

Summary

Samenvatting

Dankwoord

Curriculum Vitae

List of publications 


\section{Abbreviations}

\begin{tabular}{|c|c|}
\hline$\beta$-TG & $\beta$-thromboglobulin \\
\hline $\mathrm{Ag}^{0}$ & metallic silver \\
\hline $\mathrm{Ag}^{+}$ & silver ions \\
\hline $\mathrm{AgBr}$ & silverbromide \\
\hline $\mathrm{AgNP}$ & silver nanoparticle \\
\hline AT III & antithrombin III \\
\hline BMA & n-butyl methacrylate \\
\hline BSA & bovine serum albumin \\
\hline BSI & bloodstream infection \\
\hline CABG & coronary artery bypass grafting \\
\hline Carmeda ${ }^{\circledR}$ & Carmeda BioActive Surface ${ }^{\circledR}$ \\
\hline CHSS-CVC & $\begin{array}{l}\text { central venous catheter with chlorhexidine-silver } \\
\text { sulfadiazine coating }\end{array}$ \\
\hline CI & Confidence Interval \\
\hline $\mathrm{CPB}$ & cardiopulmonary bypass \\
\hline CRBSI & catheter-related bloodstream infection \\
\hline CTAD & citrate-theophylline-adenosine-dipyridamole \\
\hline CVC & central venous catheter \\
\hline $\mathrm{D}$ & dendritic \\
\hline DMSO & dimethylsulfoxide \\
\hline EDTA & ethylenediaminetetraacetic acid \\
\hline ELISA & enzyme-linked immunosorbent assay \\
\hline ELS & extracorporeal life support \\
\hline FS & fully spread \\
\hline $\mathrm{Hb}$ & hemoglobin \\
\hline HEPES & 4-(2-hydroxyethyl)-1-piperazineethanesulfonic acid \\
\hline HITTS & $\begin{array}{l}\text { heparin-induced thrombocytopenia and thrombosis } \\
\text { syndrome }\end{array}$ \\
\hline ICP-OES & inductively coupled plasma-optical emission spectroscopy \\
\hline $\mathrm{ICU}$ & intensive care unit \\
\hline $\operatorname{Ig} G$ & immunoglobulin $G$ \\
\hline LDH & lactate dehydrogenase \\
\hline MPO & myeloperoxidase \\
\hline MR-CVC & central venous catheter with minocycline-rifampin coating \\
\hline NPVP & $\begin{array}{l}\text { [poly(4-vinylpyridine)-co-poly(4-vinyl-N-hexylpyridinium } \\
\text { bromide)] }\end{array}$ \\
\hline NVP & $\mathrm{N}$-vinylpyrrolidinone \\
\hline Oligon-CVC & central venous catheter treated with oligon \\
\hline ON & overnight \\
\hline PBS & phosphate buffered saline \\
\hline PET & poly(ethylene terephtalate) \\
\hline
\end{tabular}


PMEA poly(2-methoxy-ethylacrylate)

PRP

PTCA

PTFE

PU

PVC

$\mathrm{R}$

$\mathrm{S}$

SD

sem

SEM

TBS ${ }^{\circ}$

TCC

TGT $_{\text {lag }}$

UFH

XPS platelet-rich plasma

percutaneous transluminal coronary angioplasty

poly(tetrafluoroethylene)

poly(urethane)

poly(vinylchloride)

round

spread

spread dendritic

standard error of the mean

scanning electron microscopy

Trillium ${ }^{\circledR}$ BioSurface

terminal complement complex

thrombin generation lag time

unfractionated heparin

$\mathrm{X}$-ray photoelectron spectroscopy 
Voor mïn ouders 
General introduction 
Chapter 1

$\stackrel{0}{=}$ 
Cardiovascular medical devices include catheters for blood access and blood vessel manipulation (e.g. angioplasty), stents, heart assist devices, vascular grafts, prosthetic heart valves, extracorporeal pump-oxygenator systems (used in many surgical procedures ${ }^{1,2,3}$ ), and hollow-fibre hemodialyzers to treat kidney failure. All cardiovascular devices have in common that they are in contact with the patient's blood. The duration of the contact can vary between minutes and years (i.e. the rest of the patient's life time). The blood-contacting surface of any cardiovascular device is critical, especially since the contact can lead to the formation of thrombus that can adhere to the surface ${ }^{4,5,6}$, or can detach from the surface, to embolize a blood vessel downstream ${ }^{7,8,9,10,11}$. The majority of the cardiovascular biomaterials are polymers ${ }^{12,13,14}$. These materials are available in a wide range of physical and mechanical properties ${ }^{14}$. A distinct advantage of polymers is, furthermore, that they can easily be manufactured into products with a desired shape. The most common polymers used in cardiovascular medical devices are: poly(tetrafluoroethylene) (PTFE), poly(dimethylsiloxane), poly(propylene), poly(ethylene terephtalate) (PET), poly(urethanes) (PU), nylons, poly(ethylene); low- and high density, poly(sulfones), and poly(vinylchloride) (PVC) ${ }^{12,13,14}$. Cardiovascular biomaterials and the devices manufactured from them have revolutionized modern healthcare during the last 5 - 6 decades, and these developments are still ongoing. The global market for cardiovascular biomaterials/devices is expected to reach 20.7 billion US\$ in 2014, with an annual growth rate of $12.5 \%$, thereby surpassing all other segments in the biomaterials market ${ }^{15}$.

This thesis deals primarily with the problem of thrombogenicity. According to the Williams Dictionary of Biomaterials 1999, "thrombogenicity" is defined as the property of a material which induces and/or promotes the formation of a thrombus ${ }^{16}$. Blood contacting biomaterials tend to ignite different coagulation mechanisms, and this can have devastating effects. Many cardiovascular devices are considered to be "safe" only when anticoagulant drugs are used ${ }^{17,18,19}$. The use of such drugs, however, has important implications, particularly an elevated risk for internal bleeding $20,21,22,23,24,25,26,27$. This thesis concentrates on two of such situations: thrombus formation in devices for prolonged cardio-pulmonary bypass systems, and thrombus formation at the surface of central venous catheters.

\section{Coagulation occurring at artificial surfaces}

Within seconds following contact between blood and an artificial material, plasma proteins adsorb onto the material's surface ${ }^{28}$. The adsorption process induces conformational changes in some plasma proteins 29,30,31,32, resulting in partial denaturation and exposure of reactive domains $29,30,31,32,33$. These are initial events in the triggering of thrombotic and inflammatory 
responses $28,29,30,31,33,34$. Among the adsorbed proteins are coagulation factor XII, fibrinogen, Immunoglobulin $\mathrm{G}(\mathrm{IgG})$ and complement factor C3b ${ }^{28,29,30,31,33,34}$. Conformational changes in coagulation factor XII, leading to its activation, trigger the coagulation cascade and fibrin formation via the intrinsic pathway ${ }^{31}$. In addition, recent data show that proteases of the coagulation cascade can have C3 and C5 convertase activity, leading to complement activation ${ }^{35}$. Conformational change in adsorbed fibrinogen exposes reactive sites which facilitate platelet adhesion via the glycoprotein IIb/IIIa complex 34,36,37,38. Adsorbed IgG can activate the complement pathway in the vicinity of the bloodmaterial interface ${ }^{33}$. Subsequent response amplification via the alternative pathway generates large amounts of $\mathrm{C} 3 \mathrm{~b}$ which also can be adsorbed and further catalyzes downstream complement activation ${ }^{33}$.

C3a and C5a, both products of the complement cascade, display anaphylactic and chemotactic activity and elicit proinflammatory responses in neutrophils and monocytes, with cytokines, vasoactive substances and cellsignalling molecules ${ }^{39}$. Eventually, the thrombotic and inflammatory responses lead to formation of microembolic particles of fibrin, platelet aggregates, fat and other matter ${ }^{40}$. These microembolic particles can become trapped in small arteries and capillary networks of the patient's body. The damaging effects of obstructed blood flow, cytokines and inflammatory responses of neutrophils and monocytes can then disturb organ function. Especially in the brain this can have serious complications ${ }^{7,8,9,10,11}$. On the other hand, blood clot formation also compromises performance of the artificial device. Thrombotic obstruction can for example lead to oxygenator failure in CPB set-ups, leading to necessary device replacement ${ }^{4,5,6}$.

\section{Cardiopulmonary bypass systems}

The development of cardiopulmonary bypass (CPB) systems, also known as heart-lung machines, has been a milestone in the field of cardiac surgery ${ }^{41,42,43,44}$ because of the possibility to artificially take over cardiac and pulmonary function. CPB is still a necessity for cardiac surgery that requires opening of the heart, while maintaining sufficient blood flow to the rest of the body. It is now routine for surgeons to operate on the heart in an environment without movement and without blood ${ }^{45}$. The heart-lung machine is a standard support system during coronary artery bypass grafting (CABG) and valve surgery. In the Netherlands, nearly 17,000 patients had open heart surgery in $2009^{46}$ and this number is not likely to decrease. Firstly, the contribution of off-pump procedures - without CPB - is now steady around $15 \%{ }^{47}$ and secondly, the new contribution of minimal invasive procedures (without CPB), will most likely be compensated by the overall increase of procedures in patients at older ages ${ }^{48,49}$. 
More recently, refinement of the heart-lung machine and the growing experience with longer lasting support has raised the interest to expand CPB technology outside the operating room, as a bridge-to-recovery for patients with severe heart and/or respiratory failure or as a bridge to transplant ${ }^{50,51}$. These applications are usually termed as extracorporeal life support (ELS). In these settings, the patient is typically connected to the heart lung machine for an extended period of time, which may range from days to even weeks. Patients requiring prolonged ELS usually suffer from critical, life-threatening conditions with a high degree of mortality. When all conventional treatments have failed, connection to the ELS system for several days or weeks is often a last option to support the vital functions throughout the primary disease process. On the other hand, prolonged extensive blood contact with the artificial surfaces of the ELS system opposes a secondary burden for the already severely diseased individual.

The heart-lung machine of today is a highly sophisticated device and contains three basic parts: a blood pump, an oxygenator and a set of tubing circuitry. The blood pump sustains blood flow (either non-pulsatile or pulsatile), and the oxygenator allows gas exchange between blood and air. These oxygenators are artificial lungs containing two compartments, separated by a gas-permeable membrane. Blood flows through one compartment while a gas mixture is pumped through the other compartment. The oxygenator contains a relatively large surface area $\left(1.35 \mathrm{~m}^{2}-2.5 \mathrm{~m}^{2}\right)$ of gas-permeable membrane to attain high levels of gas exchange ${ }^{52}$.

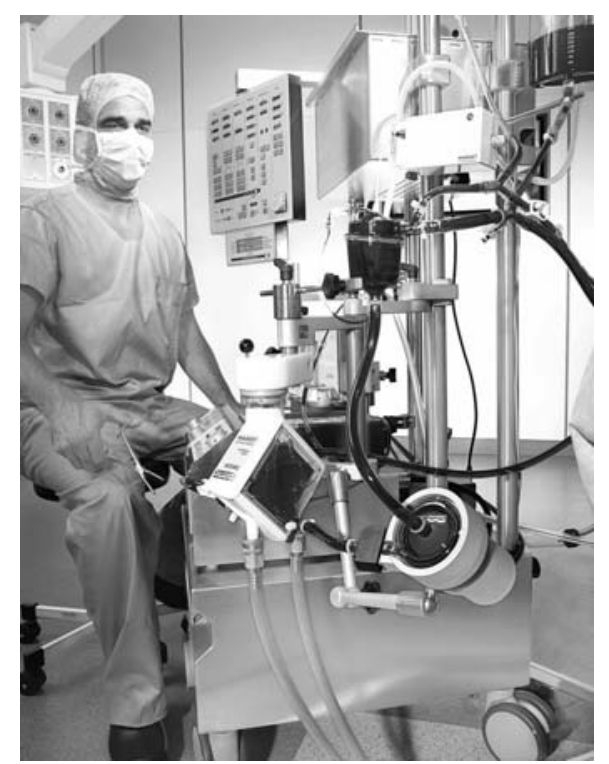

Figure 1. Picture of a typical CPB set-up. Depicted are the oxygenator (lower left, square-formed device), the blood pump (lower right, circular device). Blood is guided from the patient towards, from and between these devices through an extensive set of tubing. Note the large amount of blood present in the tubing and different components of the system ${ }^{53}$. 
To prevent the occurrence of thrombus formation on such a large surface area as the inner surface of the cardiopulmonary bypass circulatory system is an extraordinary challenge. Compared to other types of blood-contacting devices, the blood-material interaction involves a larger surface area and a longer duration. For almost six decades now, the cornerstone of measures to prevent material-induced thrombosis during CPB or ELS, is aggressive anticoagulation therapy. Unfractionated heparin (UFH) is thereby the standard anticoagulant of choice ${ }^{54,55}$. Its anticoagulant effect occurs fast after administration and can rapidly be terminated by administration of protamine sulphate ${ }^{55}$. There are, however, some important side effects. Because of its chemical structure (a macromolecular complex consisting of a core protein with a variable number of mucopolysaccharide chains) UFH has a heterogeneous molecular size, resulting in variable pharmacokinetic and pharmacodynamic effects ${ }^{55}$. The anticoagulant effect of UFH is of short duration and it has a variable circulating half-life, which makes appropriate dosing difficult 55. Therefore, it is of great importance to frequently monitor the anticoagulated state of the patient during $\mathrm{CPB}{ }^{55}$. Another disadvantage of UFH is its high antigenic potential ${ }^{55}$. In $30-50 \%$ of patients UFH triggers an antibody response and in 1-3\% of patients this results in heparin-induced thrombocytopenia and thrombosis syndrome (HITTS) ${ }^{55}$. Dangerous complications can clinically manifest themselves as venous or arterial thrombosis and, therefore, hold a considerable risk for limb ischemia, ischemic stroke or acute graft closure ${ }^{56,57}$. The HITTS syndrome has a high mortality rate, approaching 40\% ${ }^{56,58}$. Besides HIT'TS, heparin administration causes reduced platelet count by $20-30 \%{ }^{59}$. This, in combination with the anticoagulant effect of heparin, results in increased risk for bleeding complications, intra- and postoperative blood loss 55,58,60,61. Other anticoagulants proposed to replace heparin are for example lepirudin, desirudin, bivalirudin or argatroban, all direct thrombin inhibitors ${ }^{55,58,62}$.

An important part of the efforts to refine CPB technology has been dedicated to improve the interface between blood and the material surfaces, leading to lower risks of thrombus formation and need for lesser aggressive anticoagulation protocols and their associated complications ${ }^{17,18}$. A strategy that has yielded considerable progress in this respect was the development of coatings to make material surfaces more blood-compatible. This has resulted in a number of coating formulations for ELS systems that are nowadays clinically available under the term blood compatible or hemocompatible coatings ${ }^{63}$. A subset of these coatings is based on heparin, to mimic the naturally occurring glycocalix anticoagulant function by the molecule heparan sulphate ${ }^{63,64,65,66}$. Differences between these heparin coatings lie in the chemical bonding type used to attach

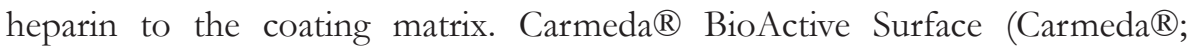

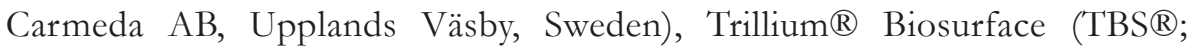
Medtronic Inc., Minneapolis, MN, USA) and Corline ${ }^{\circledR}$ (Corline Systems AB, Uppsala, Sweden) use covalent bonding ${ }^{63,66}$. Duraflo II ${ }^{\circledR}$ heparin coating 
(Bentley Laboratories Division, Baxter Healthcare Corp., Irvine, CA, USA) uses ionic bonding ${ }^{63}$, and BioLine Coating ${ }^{\circledR}$ (MAQUET GmbH \& Co. KG, Rastatt, Germany) uses both ionic and covalent bonds ${ }^{63}$. It is assumed that covalent heparin coupling offers some advantages over ionic bonding. Establishment of a covalent bond at the end of the linear heparin molecule, the so-called endpoint attachment, leaves its active site better accessible for antithrombin III (AT III). This way, the endpoint attached heparin molecules extend in the liquid phase and are able to exert their anticoagulant effect on circulating blood components. Additionally, the higher stability of a covalent bond versus an ionic bond is assumed to better prevent leaching of heparin into the circulation and loss of function of the coating.

Randomized controlled clinical trials show better outcome after open heart surgery with $\mathrm{CPB}$ using coated ECC systems versus uncoated systems $18,19,67,68,69,70,71$. Until today, however, these materials have not succeeded yet in completely replacing the need for aggressive anticoagulation therapy during CPB procedures ${ }^{17,18,19,71}$. Furthermore, fine-tuning anticoagulation therapy to achieve minimal thrombotic events without increasing the risk for bleeding complications remains difficult 20,21,22,23,24,25,26,27. The prevention of thrombotic complications in patients requiring long-term ELS remains even a bigger challenge $20,21,22,23,24,25,26,27$. Nardell et al. conducted a retrospective analysis to identify risk factors for bleeding in 145 pediatric post-cardiotomy patients requiring ELS. In this study, both thrombocytopenia and duration of $\mathrm{CPB}$ were identified as risk factors for bleeding complications and both these risk factors could be attributed mostly to blood-surface contact in the ELS system. It was postulated that contact-induced activation of coagulatory and inflammatory responses results in consumption of clotting factors and platelets, which increases the risk for bleeding ${ }^{72}$. According to the Extracorporeal Life Support Registry Report 2008 for neonatal and pediatric cardiac cases, surgical site bleeding was the second most common complication during ELS. The most common mechanical complications in these patient groups occurred in the oxygenator, harbouring the largest surface area for blood-material contact, and, blood clot formation throughout the ELS system was largely responsible for these events ${ }^{73}$. Brogan and colleagues analyzed the international database of the Extracorporeal Life Support Organization and retrospectively compared cases of surviving ( $\mathrm{n}=741$ ) and non-surviving $(\mathrm{n}=732)$ adult patients with severe respiratory failure between 1986 and 2006. Blood clot formation within the ELS circuit occurred frequently in both the surviving and non-surviving group, respectively in 17\% and $8 \%$ of patients. Surgical site bleeding also frequently occurred in both groups and was significantly $(\mathrm{p}<0.001)$ higher in the nonsurviving group $(36 \%)$ than in the surviving patients $(24 \%)^{74}$.

The first part of this thesis focuses on these thrombotic problems encountered during long-term use of heart-lung machinery, even in presence of a blood compatible coating. Further research to develop improved blood- 
compatible coatings could decrease the risk for thrombotic complications during ELS and simultaneously allow less aggressive anticoagulation, minimizing the risk for bleeding complications.

\section{Central venous catheters}

A central venous catheter (CVC) is, as the name explains, a catheter type inserted into large veins, such as the subclavian, femoral, or internal jugular vein. CVCs are used worldwide for the management of critically ill patients. They are indispensable tools for rapid access to the bloodstream for the administration of drugs, parenteral nutrition, blood products, for measurement of hemodynamic variables, or for withdrawal of blood samples ${ }^{75}$. In the USA, an estimated number of more than five million patients per year require a $\mathrm{CVC}^{75,76}$. Among the patient groups in which CVCs are most frequently used are cancer patients, especially those with hematologic malignancies, patients in intensive care units (ICUs) and hemodialysis patients. Also after cardiac surgery patients may get a central line during the first week post-operatively.

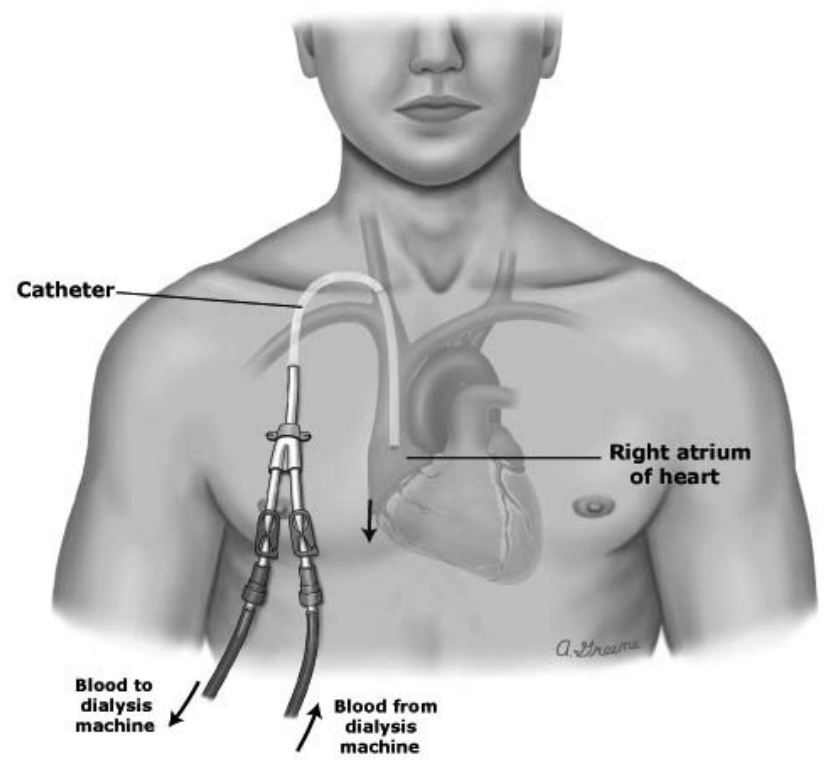

Figure 2. Picture of a central venous catheter inserted in the right jugular vein. Note the short distance between the venous bloodstream and the skin surface at the insertion site ${ }^{77}$.

Unfortunately, several complications frequently occur with the use of CVCs. These complications can roughly be divided in three categories: mechanical, thrombotic and infectious complications ${ }^{75}$. Mechanical complications, such as blood vessel perforation, hematoma and pneumothorax, fall outside the scope of this thesis and will not further be discussed. 
It is believed that infectious complications of CVCs are interrelated with thrombotic complications. Evidence for this comes from several studies ${ }^{78,79,80,81}$. This is the reason why these complications are shortly explained here. Infectious complications arise with 3 to $8 \%$ of inserted catheters and account for approximately 80,000 catheter-related bloodstream infections (CRBSIs) per year in ICUs throughout the USA, which makes them the primary cause of nosocomial bloodstream infections (BSIs) in ICUs ${ }^{76}$. Combined with CRBSIs occurring outside the ICU, a total number of 250,000 CRBSIs are estimated to occur annually. CRBSIs cause a significant burden in terms of morbidity, mortality and healthcare costs. In terms of morbidity, the occurrence of CRBSIs usually results in prolonged hospital stay and additional therapy ${ }^{82}$. The exact burden in terms of mortality is still unclear. Estimates for the attributable mortality of CRBSIs range from 0 to $35 \%$ depending on the degree of control for severity of illness ${ }^{76,83}$. Per infection, the costs related to additional care are estimated to be in the range of $\$ 25,000-\$ 30,000{ }^{76,83}$. This results in attributable annual health care costs ranging from $\$ 296$ million to $\$ 2.3$ billion ${ }^{76,83}$. In addition, Medicare, the United States government's health insurance program for elderly and disabled Americans, stopped reimbursement of the costs for preventable hospital-acquired conditions, among which are catheter-related infections ${ }^{84}$.

Currently, a combination of different measures is implemented in clinical practice to reduce catheter-related infections. Standard operation procedures are applied to ensure hygiene and minimize contamination during catheter insertion, catheter manipulation and catheter care. Such protocols include for example hand hygiene, wearing sterile clothing (gloves, mask, cap, gown), use of a large sterile drape and skin antisepsis at the insertion site. The catheter insertion site is protected with dressings permeable to water vapour to prevent trapping of moisture and creation of an environment favourable for microbial growth. Several types of CVC are also available that vary in their risk for CRBSIs ${ }^{76}$. The subclavian vein is preferentially chosen as the catheter insertion site since it was shown that this location holds the lowest risk for catheter colonization. To further minimize the risk for catheter-related infections, ultrasound guidance can be used to facilitate catheter insertion and can decrease the number of puncture failures, complications and the catheter insertion time. Another measure to prevent catheter-related infections is the use of antimicrobial lock solutions. These are solutions containing antibiotics or antiseptics and an anticoagulant, which are injected into the catheter lumen and locked in there until the next use of the catheter ${ }^{85,86}$.

The most direct approach to reduce CRBSIs is probably to coat catheters with antibiotics or antiseptics to prevent adhesion and/or survival of microorganisms on the catheter surface. Several combinations of antibiotics or antiseptics have been used in coatings to prevent microbial colonization. The 
best-known examples of antimicrobial CVCs are those impregnated with chlorhexidine and silver sulfadiazine (CHSS-CVCs), CVCs coated with minocycline and rifampin (MR-CVCs), oligon treated catheters (oligon-CVCs) and CVCs impregnated with silver ${ }^{76,87,88,89}$. These so-called antimicrobial coatings have been evaluated in numerous clinical studies for their efficacy in reducing catheter colonization and CRBSIs ${ }^{88,89}$. Whether CVCs that have been made antimicrobial effectively succeed in reduction of catheter colonization and CRBSIs, is, however, still a matter of debate ${ }^{90,91,92}$. The methodological quality of studies comparing anti-infective catheters with standard catheters is generally poor ${ }^{90,92}$. Furthermore, recent economic analyses doubt about the costeffectiveness of implementation of antimicrobial coatings on CVCs ${ }^{90,91,92}$. Researchers therefore continue their efforts to develop more effective antimicrobial coatings.

Recent developments in chemical engineering have resulted in the ability to create silver nanoparticles (AgNPs), powders of non-ionic silver with a diameter of $100 \mathrm{~nm}$ or less. A remarkable property of such nanopowders is their extremely large surface-to-volume ratio 93. Furthermore, AgNPs have been shown to exert antibacterial effects that are thought to result from oxidation and subsequent appearance of chemisorbed silver ions at the nanoparticle surfaces ${ }^{94}$. This makes nanosilver an attractive candidate for use in antimicrobial catheter coatings. Because of the surface-to-volume ratio, AgNPs used in coatings could form a reservoir for long-term elution of antimicrobial silver ions $\left(\mathrm{Ag}^{+}\right){ }^{95,96}$. This represents an important advantage compared to coatings with other forms of silver, like silver salts, of which the counter-ion may cause problems upon dissociation in vivo ${ }^{95}$. The potential of AgNPs to achieve long-term antimicrobial effects through elution of $\mathrm{Ag}^{+}$, is increasingly being explored in antimicrobial coatings for CVC materials ${ }^{97,98,99,100,101}$. At the beginning of this PhD-project, however, nothing was known about the blood compatibility of nanosilver-based coatings, what brings us back to the main topic of this $\mathrm{PhD}$-thesis: thrombogenicity of blood-contacting materials.

Thrombotic complications are thought to arise in $2-26 \%$ of patients with central lines ${ }^{75}$. As described earlier, contact between the blood and an artificial surface can disturb the blood's hemostatic systems resulting in thrombus formation and, possibly, embolisation. Beside blood-material interactions, a number of other factors increase the risk for thrombus formation in patients with CVCs. Among these factors is the catheter insertion site. It was shown previously that the occurrence of catheter-related thrombosis was lowest in patients with subclavian vein catheterization compared to patients with femoral and internal jugular catheterization. Another factor that increases the risk for catheter-related thrombosis is inherent to the patient's underlying disease process or its treatment. Normal blood homeostasis can be severely disturbed in patients with hematologic malignancies. Furthermore, also chemotherapy influences blood homeostasis ${ }^{75}$. The second part of this thesis will address the 
blood-compatibility of nanosilver-based coatings. These materials are increasingly being investigated for their potential to make surfaces more antimicrobial. Acceptable blood compatibility is, however, a prerequisite to be suitable for use on CVCs without increasing the risk for both thrombotic and infectious complications.

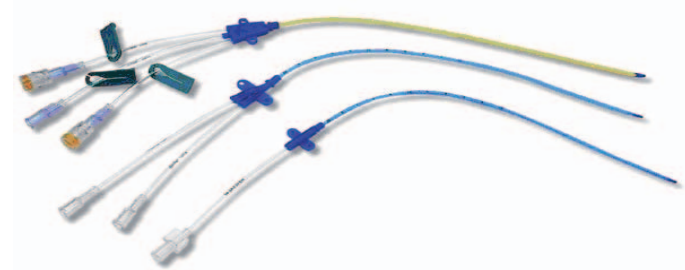

Figure 3. A set of some typical CVCs. Depicted are a triple-lumen CVC (upper CVC), a double-lumen CVC (middle CVC), and a single-lumen CVC (lower CVC) ${ }^{102}$.

\section{In vitro methods to assess thrombogenicity and antimicrobial activity of biomaterials}

\section{Evaluation of blood-coating contact under flow}

A first step in the development of blood compatible coatings is the in vitro evaluation of interactions between the blood and the coating surface. Ideally, the in vitro test model should include blood-coating contact under flow. Several of such models have been described so far.

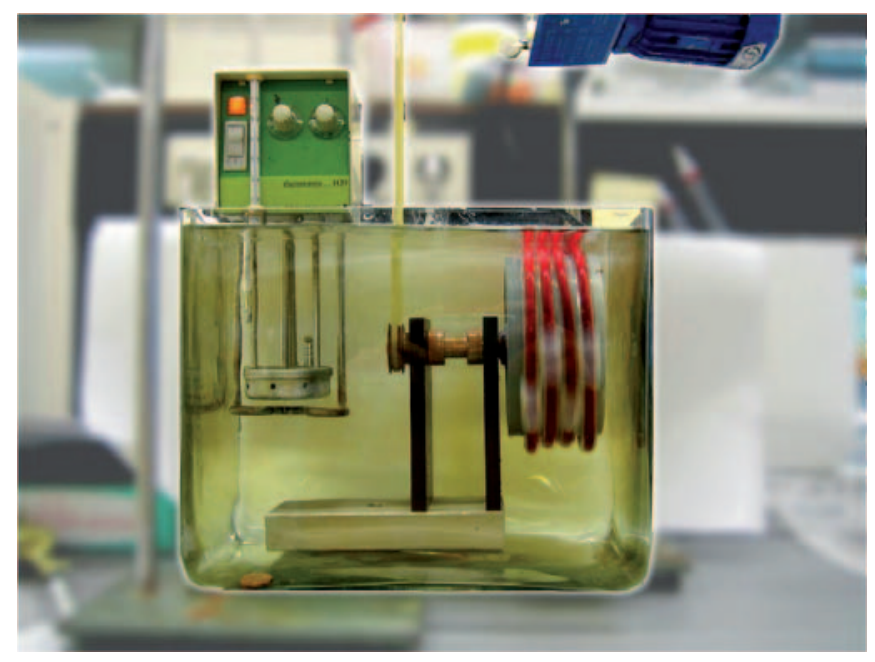

Figure 4. Picture of the modified Chandler loop set-up. Blood-filled tubing loops are mounted on a wheel of which the axis is stabilized by a heavy construct. The total construction is placed in a water bath heated to $37^{\circ} \mathrm{C}$ and can be rotated at different speeds. 
The model that was used in this thesis to evaluate blood-coating contact under flow is a modified Chandler loop system. Tubing with or without coating is filled with fresh whole blood and closed with a plastic sleeve to create circular loops. In each loop, a small air bubble is left before closing the loops. The closed tubing loops are mounted on a wheel that is subsequently placed in a water bath. The wheel can be rotated at different speeds. Together with the air bubble left in each tubing loop, the rotational movement creates a flow-like situation at the blood-coating interface. Figure 4 shows the typical modified Chandler loop setup that was used throughout this work.

The modified Chandler loop model has been used by several research groups as an in vitro model to evaluate interactions between the blood and artificial surfaces under flow ${ }^{103,104,105,106,107}$. Relatively small amounts of sample material are needed to create tubing loops and the model allows multiple experiments to be performed simultaneously. In addition, the amount of blood required to fill a tubing loop is relatively small. This ensures that all experiments performed during one run can be executed with identical blood, a feature that is very important for the evaluation of hemocompatibility. The composition of human whole blood, its tendency to clot and multiple other parameters can fluctuate over time, for example due to a person's diet (e.g. alcohol use), use of medication (e.g. aspirin) or inflammatory processes occurring in the body. Also between donors, considerable variation can exist for several blood parameters. To exclude such additional variables, it is thus important to perform experiments with blood from a single donor, collected at one time point.

The presence of a small air bubble inside each tubing loop is a necessity to ensure mixing during rotation of the wheel. Consequently, blood-air contact is occurring standard during experiments in the modified Chandler loop model. Effects of blood-air contact have been described and include complement activation ${ }^{108}$. To minimize artefacts due to blood-air contact, the size of the air bubble inside tubing loops can be kept small. The remaining blood-air contact could be compared to the blood-air contact inside an oxygenator of a real-life CPB set-up.

Several authors reported using a closed-loop CPB model in which whole blood was circulated through an oxygenator and tubing circuitry by use of a blood pump ${ }^{109,110,111,112}$. In principle such a model includes all parts of a clinically used CPB system and allows blood flow rates comparable to the in vivo situation. The relatively large amount of blood required to fill a closed-loop set up with both an oxygenator and tubing circuitry seriously limits the ability to perform multiple experiments in parallel with identical blood. Also, it is often not feasible to acquire oxygenators coated with experimental coatings. These devices are usually only available with coatings that are already in clinical use. 


\section{Evaluation of the thrombogenicity of coatings}

Throughout this thesis, the thrombogenicity of various coatings was measured using a modified version of the thrombin generation lag time ( $\left.\mathrm{TGT}_{\mathrm{lag}}\right)$ measurements described by Hemker, et al. ${ }^{113}$ The modifications applied to Hemker's original TGT model are based on the fact that, during blood-surface contact, activation of the coagulation cascade occurs via the intrinsic pathway. As described earlier (vide supra), the adhesion of plasma proteins on bloodcontacting artificial surfaces, can result in conformational changes and exposure of active sites of the adhered proteins. Among these plasma proteins are proteins of the coagulation cascade, such as factor XII. The TGT method performed throughout this thesis, therefore does not involve addition of tissue factor at the start of the experiments. As an alternative, the experimental milieu in the modified experiments contains samples of coated tubing as the coagulation activating agents.

In short, whole blood is collected on citrate, a calcium chelating anticoagulant. Platelet-rich plasma (PRP) is isolated after centrifugation. Prior to the experiments, a thrombin-specific, fluorogenic substrate (Z-Gly-Gly-ArgAMC) is added to the PRP. The graph below presents two thrombin generation curves.

a

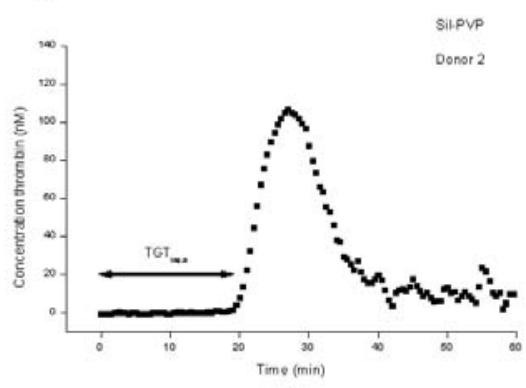

b

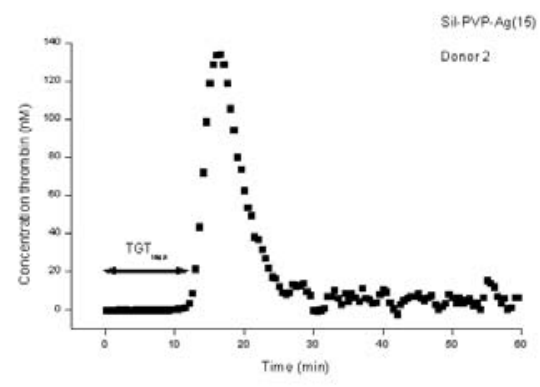

Figure 5. Thrombin generation curves measured for two different catheter samples (Sil-PVP (a) and Sil-PVP-Ag(15) (b)). Each curve represents the concentration of thrombin (y-axis) at each time point throughout the experiment (x-axis). Both experiments were performed simultaneously with the same PRP, freshly isolated from the same donor (Donor 2). Notice how the time span between the onset of the experiment and the sudden rise in thrombin levels ( $\mathrm{TGT}_{\mathrm{lag}, \mathrm{a}}$ and $\mathrm{TGT}_{\mathrm{lag}, \mathrm{b}}$ ) can differ for different catheter samples.

Each curve represents the concentrations of thrombin in one specific well throughout the experiment. At first, no thrombin is present yet, which is represented by the lag time in the thrombin generation curve. Following activation of the intrinsic coagulation pathway, thrombin is formed explosively, which is seen in the curve as a steep increase in the thrombin concentration to 
a maximum. Shortly after the onset of thrombin formation, inhibitory mechanisms arrest thrombin formation and thrombin activity, which is represented as a decline in thrombin concentration rapidly following the obtained maximum thrombin concentration. In particular the $\mathrm{TGT}_{\mathrm{lag}}$, at the start of the experiment, is of importance to characterize the thrombogenicity of blood-contacting materials. It represents the time period during which blood or plasma contacts the material's surface without activation of the intrinsic coagulation pathway.

This modified TGT method was shown to be a sensitive method to discriminate between different materials in terms of their surface thrombogenicity ${ }^{114}$. The use of a fluorogenic substrate and fluorescence tracing throughout the experiment makes the TGT experiments far less timeconsuming than the subsampling method. Furthermore, the method requires only small amounts of sample material and very low amounts of blood. Multiple experiments can therefore be performed in parallel with identical blood. A limitation of the modified TGT method is the absence of flow. A variant of the currently described method, in which TGT was measured under flow, was reported by Aldenhoff, et al., but was not feasible for the work presented in this thesis ${ }^{115,116}$. The inclusion of flow in the model requires tubing samples with only a very small diameter. This complicates the coating process.
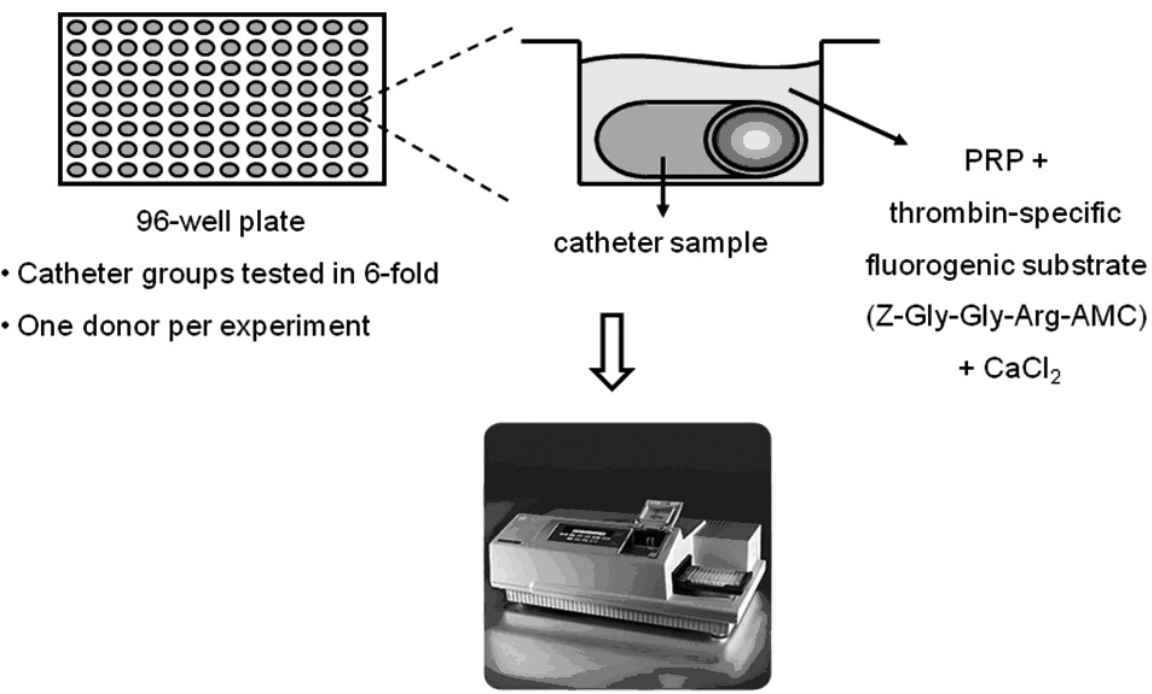

Fluorescence tracing: over $1 \mathrm{~h}$, every $30 \mathrm{~s}$;

$37^{\circ} \mathrm{C}$ and shaking conditions

Figure 6. Scheme of the experimental protocol for the thrombin generation experiments. The use of a 96-well plate allows different catheter samples to be tested simultaneously with identical PRP, freshly isolated from a single donor. 


\section{Evaluation of the antimicrobial activity of coatings}

An initial event in the pathogenesis leading to CRBSI involves microbial colonization of the intravascular catheter surfaces. According to a meta-analysis by Rijnders et al., the incidence of tip colonization correlates well with the incidence of CRBSI ${ }^{117}$. To prevent development of CRBSI, antimicrobial coatings should prevent colonization of the catheter surfaces with microorganisms. Throughout this thesis, the experimental set-up to analyse a catheter material's ability to prevent microbial colonization was based on the semiquantitative catheter segment culture, also known as roll-plate analysis, first described by Maki and colleagues ${ }^{118,119}$.

Roll-plate analysis has been in clinical use for several decades already as a generally accepted semi-quantitative method to diagnose CRBSI ${ }^{119}$. A metaanalysis by Safdar, et al. reported an overall sensitivity of 0.85 (Confidence Interval (CI), 0.81 to 0.89$)$ and an overall specificity of 0.82 (CI, 0.80 to 0.84$)^{119}$. In patients with suspected CRBSI the intravascular end of the catheter explant is rolled over an agar plate. During the rolling process, bacteria present at the catheter surface can be transferred to the agar. Subsequent, overnight culture of the agar plate yields colonies that can be used to determine the identity of the colonizing species. A cut-off value of $\geq 15$ colony forming units is used to discriminate between CRBSI or catheter colonization ${ }^{119}$.

The roll-plate analysis is only a semi-quantitative method. Another, more quantitative method to determine catheter colonization is quantitative sonication ${ }^{120}$. However, because even a rigorous method like sonication does not ensure complete removal of all bacteria from the catheter, roll-plate analysis, the golden standard, was the method of choice throughout this thesis to evaluate catheter colonization. The roll-plate analysis is also a simple technique and therefore easy to perform.
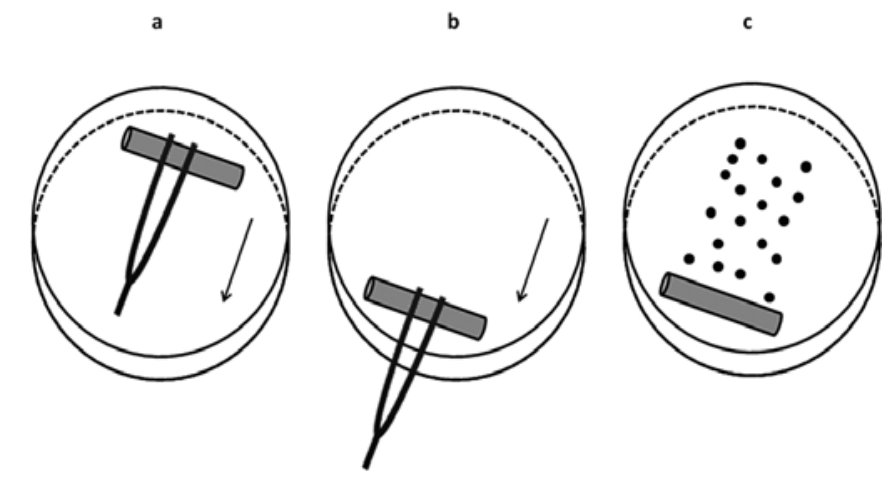

Figure 7. Scheme of the roll-plate analysis protocol. (a) and (b): Following incubation in a suspension of bacteria, catheter samples are placed on an agar plate and rolled to the opposite side with a sterile tweezers. During the rolling process bacteria can detach from the catheter surface and are transferred to the agar plate. (c): Overnight incubation at $37^{\circ} \mathrm{C}$ allows viable bacteria to form colonies on the agar plate. The amount of colonies formed gives an indication of the number of viable bacteria present on the catheter surface after incubation in bacterial suspension. 


\section{Outline of the Thesis}

Chapter 2 came forth from the engineering of surface coatings with long-term blood-compatibility. Using the well-known Chandler loop system, a systematic evaluation of 2 commercial CPB tubings, each with a hemocompatible coating, has been performed under conditions of relatively long blood-contact times. An uncoated tube was used as a control. The experiments comprised testing during $5 \mathrm{~h}$ with fresh whole blood from 4 different human donors. Essential blood parameters were measured and the luminal surfaces of the tubings were studied with scanning electron microscopy and thrombin generation measurements.

Chapter 3 describes the analysis of two nanosilver coatings with respect to their antimicrobial properties and blood compatibility. The coating matrices without AgNPs served as control materials, as well as state-of-the-art clinically available antimicrobial and blood compatible coatings. Additional series of titration experiments were performed to gain insights in the mechanisms behind the nanosilver coatings' effects.

In Chapter 4 the blood compatibility of an antimicrobial silverbromide $(\mathrm{AgBr})$ nanocomposite coating was investigated with respect to both platelets and the coagulation cascade. These materials were previously shown to display strong antimicrobial effects, however, nothing was known about their blood compatibility.

Chapter $\mathbf{5}$ originated from the hypothesis that effective antimicrobial CVC coatings should display both antimicrobial activity and acceptable blood compatibility. Described is the engineering of dual-action coatings embedded with both AgNPs and heparin, and control materials embedded with either AgNPs or heparin, or neither of these two. Following the engineering of these materials, a first series of experiments were performed to characterize the coatings' antimicrobial properties and blood compatibility. 


\section{References}

1 Christiansen, S. \& Autschbach, R. Results and treatment strategy for patients undergoing emergent coronary artery bypass grafting. Ann Thorac Cardiovasc Surg 16, 168-173 (2010).

2 Ferrari, E., Stalder, N. \& von Segesser, L. K. On-pump beating heart coronary surgery for high risk patients requiring emergency multiple coronary artery bypass grafting. J Cardiothorac Surg 3, 38 (2008).

3 Miyahara, K. et al. On-pump beating-heart coronary artery bypass grafting after acute myocardial infarction has lower mortality and morbidity. J Thorac Cardiovasc Surg 135, 521-526 (2008).

4 Lehle, K. et al. Efficiency in extracorporeal membrane oxygenation-cellular deposits on polymethylpentene membranes increase resistance to blood flow and reduce gas exchange capacity. AS AIO J 54, 612-617 (2008).

5 Wendel, H. P., Philipp, A., Weber, N., Birnbaum, D. E. \& Ziemer, G. Oxygenator thrombosis: worst case after development of an abnormal pressure gradient--incidence and pathway. Perfusion 16, 271-278 (2001).

$6 \mathrm{Yu}, \mathrm{K}$. et al. Clinical Evaluation of Two Different Extracorporeal Membrane Oxygenation Systems: A Single Center Report. Artif Organs (2011).

7 Bierbach, B. et al. Emboli formation rather than inflammatory mediators are responsible for increased cerebral water content after conventional and assisted beating-heart myocardial revascularization in a porcine model. Stroke 39, 213-219 (2008).

8 Bokeriia, L. A. et al. Asymmetric cerebral embolic load and postoperative cognitive dysfunction in cardiac surgery. Cerebrovasc Dis 23, 50-56 (2007).

9 Carrier, M., Denault, A., Lavoie, J. \& Perrault, L. P. Randomized Controlled Trial of Pericardial Blood Processing With a Cell-Saving Device on Neurologic Markers in Elderly Patients Undergoing Coronary Artery Bypass Graft Surgery. The Annals of Thoracic Surgery 82, 51-55 (2006).

10 Groom, R. C. et al. Microemboli from cardiopulmonary bypass are associated with a serum marker of brain injury. J Extra Corpor Technol 42, 40-44 (2010).

11 Klement, P. et al. Antithrombin-heparin covalent complex reduces microemboli during cardiopulmonary bypass in a pig model. Blood 116, 5716-5723 (2010).

12 Ravi, S., Qu, Z. \& Chaikof, E. L. Polymeric materials for tissue engineering of arterial substitutes. Vascular 17 Suppl 1, S45-54 (2009).

13 Roll, S. et al. Dacron vs. PTFE as bypass materials in peripheral vascular surgery--systematic review and meta-analysis. BMC Surg 8, 22 (2008).

14 Spijker, H. T., Graaff, R., Boonstra, P. W., Busscher, H. J. \& van Oeveren, W. On the influence of flow conditions and wettability on blood material interactions. Biomaterials 24, 4717-4727 (2003).

15 marketsandmarkets.com. Global Biomaterials Market (2009-2015). Report No. BT 1026, (2009).

16 Williams, D. F. The Williams dictionary of Biomaterials. (1999) ISBN 0-85323-921-5.

17 Fromes, Y. et al. A comparison of low vs conventional-dose heparin for minimal cardiopulmonary bypass in coronary artery bypass grafting surgery. Anaesthesia 66, 488-492 (2011).

18 Gunaydin, S. et al. Clinical and biomaterial evaluation of hyaluronan-based heparin-bonded extracorporeal circuits with reduced versus full systemic anticoagulation in reoperation for coronary revascularization. J Cardiovasc Med (Hagerstown) 10, 135-142 (2009).

19 Ranucci, M., Balduini, A., Ditta, A., Boncilli, A. \& Brozzi, S. A systematic review of biocompatible cardiopulmonary bypass circuits and clinical outcome. Ann Thorac Surg 87, 1311-1319 (2009).

20 Barrett, C. S. et al. Neurological injury after extracorporeal membrane oxygenation use to aid pediatric cardiopulmonary resuscitation. Pediatr Crit Care Med 10, 445-451 (2009). 


\section{Chapter 1}

21 Fischer, S. et al. Extracorporeal membrane oxygenation for primary graft dysfunction after lung transplantation: analysis of the Extracorporeal Life Support Organization (ELSO) registry. J Heart Lung Transplant 26, 472-477 (2007).

22 Gow, K. W. et al. Extracorporeal life support for support of children with malignancy and respiratory or cardiac failure: The extracorporeal life support experience. Crit Care Med 37, 1308-1316 (2009).

23 Ko, W. J., Hsu, H. H. \& Tsai, P. R. Prolonged extracorporeal membrane oxygenation support for acute respiratory distress syndrome. J Formos Med Assoc 105, 422-426 (2006).

24 Linden, V. et al. High survival in adult patients with acute respiratory distress syndrome treated by extracorporeal membrane oxygenation, minimal sedation, and pressure supported ventilation. Intensive Care Med 26, 1630-1637 (2000).

25 Megarbane, B. et al. Emergency feasibility in medical intensive care unit of extracorporeal life support for refractory cardiac arrest. Intensive Care Med 33, 758-764 (2007).

26 Meister, B. et al. Extracorporeal membrane oxygenation as a rescue therapy for leukaemic children with pulmonary failure. Br J Haematol 148, 126-131 (2010).

27 Mongero, L. B. et al. Extracorporeal membrane oxygenation for diffuse alveolar hemorrhage and severe hypoxemic respiratory failure from silicone embolism. Perfusion 25, 249-252; discussion 253-244 (2010).

28 Engberg, A. E. et al. Blood protein-polymer adsorption: Implications for understanding complement-mediated hemoincompatibility. J Biomed Mater Res A (2011).

29 Andersson, J., Ekdahl, K. N., Larsson, R., Nilsson, U. R. \& Nilsson, B. C3 adsorbed to a polymer surface can form an initiating alternative pathway convertase. I Immunol 168, 5786-5791 (2002).

30 Wettero, J., Bengtsson, T. \& Tengvall, P. C1q-independent activation of neutrophils by immunoglobulin M-coated surfaces. J Biomed Mater Res 57, 550-558 (2001).

31 Back, J. et al. Distinctive regulation of contact activation by antithrombin and C1-inhibitor on activated platelets and material surfaces. Biomaterials 30, 6573-6580 (2009).

32 Wu, Y., Simonovsky, F. I., Ratner, B. D. \& Horbett, T. A. The role of adsorbed fibrinogen in platelet adhesion to polyurethane surfaces: a comparison of surface hydrophobicity, protein adsorption, monoclonal antibody binding, and platelet adhesion. J Biomed Mater Res A 74, 722-738 (2005).

33 Andersson, J., Ekdahl, K. N., Lambris, J. D. \& Nilsson, B. Binding of C3 fragments on top of adsorbed plasma proteins during complement activation on a model biomaterial surface. Biomaterials 26, 1477-1485 (2005).

34 Sivaraman, B. \& Latour, R. A. The relationship between platelet adhesion on surfaces and the structure versus the amount of adsorbed fibrinogen. Biomaterials 31, 832-839 (2010).

35 Amara, U. et al. Molecular intercommunication between the complement and coagulation systems. J Immunol 185, 5628-5636 (2010).

36 Chiumiento, A., Lamponi, S. \& Barbucci, R. Role of fibrinogen conformation in platelet activation. Biomacromolecules 8, 523-531 (2007).

37 Hylton, D. M., Shalaby, S. W. \& Latour, R. A., Jr. Direct correlation between adsorptioninduced changes in protein structure and platelet adhesion. J Biomed Mater Res A 73, 349-358 (2005).

38 Koh, L. B., Rodriguez, I. \& Venkatraman, S. S. Conformational behavior of fibrinogen on topographically modified polymer surfaces. Phys Chem Chem Phys 12, 10301-10308 (2010).

39 Fung, M. et al. Inhibition of complement, neutrophil, and platelet activation by an anti-factor D monoclonal antibody in simulated cardiopulmonary bypass circuits. J Thorac Cardiovasc Surg 122, 113-122 (2001).

40 Melvin, M. E., Fissell, W. H., Roy, S. \& Brown, D. L. Silicon induces minimal thromboinflammatory response during 28-day intravascular implant testing. ASAIO J 56, 344-348 (2010). 
41 Cooley, D. A. A milestone in cardiovascular surgery. J Thorac Cardiovasc Surg 126, 12431244 (2003).

42 Daly, R. C. et al. Fifty years of open heart surgery at the Mayo Clinic. Mayo Clin Proc 80, 636-640 (2005).

43 Edmunds, L. H. Cardiopulmonary bypass after 50 years. N Engl J Med 351, 1603-1606 (2004).

44 Stoney, W. S. Evolution of cardiopulmonary bypass. Circulation 119, 2844-2853 (2009).

45 Kahn, S. R. Recurrent venous thromboembolism in men and women. N Engl J Med 351, 2015-2018; author reply 2015-2018 (2004).

46 Hamad, M. A. et al. Preoperative ejection fraction as a predictor of survival after coronary artery bypass grafting: comparison with a matched general population. J Cardiothorac Surg 5, 29 (2010).

47 Racz, M. J. et al. A comparison of short- and long-term outcomes after off-pump and onpump coronary artery bypass graft surgery with sternotomy. J Am Coll Cardiol 43, 557-564 (2004).

48 Davis, W. J., 3rd et al. Open heart surgery in patients 85 years and older. J Card Surg 19, 7-11 (2004).

49 Zingone, B. et al. Early and late outcomes of cardiac surgery in octogenarians. Ann Thorac Surg 87, 71-78 (2009).

50 Bartlett, R. H. Extracorporeal life support: history and new directions. ASAIO J 51, 487-489 (2005).

51 Baughman, K. L. \& Jarcho, J. A. Bridge to life--cardiac mechanical support. N Engl J Med 357, 846-849 (2007).

52 Issitt, R., Cumberland, T., Clements, A. \& Mulholland, J. Clinical evaluation of the Admiral $1.35 \mathrm{~m}^{2}$ hollow-fibre membrane oxygenator. Perfusion 23, 33-38 (2008).

53 AG, M. C. Rotaflow Centrifugal Pump, <http://www.maquet.com/content/Cardiopulmonary/ Documents/Brochures/ROTAFLOW_BROCHU_V06_EN_NONUS.pdf> (2010).

54 Lobato, R. L. et al. Anticoagulation management during cardiopulmonary bypass: a survey of 54 North American institutions. J Thorac Cardiovasc Surg 139, 1665-1666 (2010).

55 Yavari, M. \& Becker, R. C. Anticoagulant therapy during cardiopulmonary bypass. J Thromb Thrombolysis 26, 218-228 (2008).

56 Koster, A., Pasic, M., Bauer, M., Kuppe, H. \& Hetzer, R. Hirudin as anticoagulant for cardiopulmonary bypass: importance of preoperative renal function. Ann Thorac Surg 69, 37-41 (2000).

57 Shammas, N. W. Bivalirudin: pharmacology and clinical applications. Cardiovasc Drug Rev 23, 345-360 (2005).

58 Follis, F. et al. Argatroban as a substitute of heparin during cardiopulmonary bypass: a safe alternative? Interact Cardiovasc Thorac Surg 10, 592-596 (2010).

59 Hirsh, J. et al. Heparin and low-molecular-weight heparin: mechanisms of action, pharmacokinetics, dosing, monitoring, efficacy, and safety. Chest 119, 64S-94S (2001).

60 Fleming, G. M. et al. Maintaining the standard: a quality assurance study for new equipment in the Michigan ECMO Program. ASAIO J 53, 556-560 (2007).

61 Hanekamp, M. N. et al. Follow-up of newborns treated with extracorporeal membrane oxygenation: a nationwide evaluation at 5 years of age. Crit Care 10, R127 (2006).

62 Martin, M. E., Kloecker, G. H. \& Laber, D. A. Argatroban for anticoagulation during cardiac surgery. Eur J Haematol 78, 161-166 (2007).

63 Wendel, H. P. \& Ziemer, G. Coating-techniques to improve the hemocompatibility of artificial devices used for extracorporeal circulation. Eur J Cardiothorac Surg 16, 342-350 (1999).

64 Lawson, D. S. et al. North American neonatal extracorporeal membrane oxygenation (ECMO) devices and team roles: 2008 survey results of Extracorporeal Life Support Organization (ELSO) centers. J Extra Corpor Technol 40, 166-174 (2008). 


\section{Chapter 1}

65 Sievert, A. N., Shackelford, A. G. \& McCall, M. M. Trends and emerging technologies in extracorporeal life support: results of the 2006 ECLS survey. J Extra Corpor Technol 41, 73-78 (2009).

66 Tagarakis, G. I. \& Tsilimingas, N. B. Heparin-coated extracorporeal circulation systems in heart surgery. Recent Pat Cardiovasc Drug Discov 4, 177-179 (2009).

67 Gunaydin, S., McCusker, K. \& Vijay, V. Clinical and biomaterial evaluation of a new condensed dual-function extracorporeal circuit in reoperation for coronary artery bypass surgery. Int J Artif Organs 32, 802-810 (2009).

68 Gunaydin, S. et al. Hyaluronan based heparin free coated open and closed extracorporeal circuits for high risk coronary revascularization. J Extra Corpor Technol 42, 286-292 (2010).

69 Marcoux, J. et al. Outcomes comparison of 5 coated cardiopulmonary bypass circuits versus an uncoated control group of patients undergoing cardiac surgery. Perfusion 24, 307-315 (2009).

70 Sohn, N., Marcoux, J., Mycyk, T., Krahn, J. \& Meng, Q. The impact of different biocompatible coated cardiopulmonary bypass circuits on inflammatory response and oxidative stress. Perfusion 24, 231-237 (2009).

71 Ovrum, E. et al. Heparinized cardiopulmonary bypass circuits and low systemic anticoagulation: An analysis of nearly 6000 patients undergoing coronary artery bypass grafting. J Thorac Cardiovasc Surg 141, 1145-1149 (2011).

72 Nardell, K. et al. Risk factors for bleeding in pediatric post-cardiotomy patients requiring ECLS. Perfusion 24, 191-197 (2009).

73 Haines, N. M., Rycus, P. T., Zwischenberger, J. B., Bartlett, R. H. \& Undar, A. Extracorporeal Life Support Registry Report 2008: neonatal and pediatric cardiac cases. ASAIO J 55, 111116 (2009).

74 Brogan, T. V., Thiagarajan, R. R., Rycus, P. T., Bartlett, R. H. \& Bratton, S. L. Extracorporeal membrane oxygenation in adults with severe respiratory failure: a multi-center database. Intensive Care Med 35, 2105-2114 (2009).

75 McGee, D. C. \& Gould, M. K. Preventing complications of central venous catheterization. N Engl J Med 348, 1123-1133 (2003).

76 Frasca, D., Dahyot-Fizelier, C. \& Mimoz, O. Prevention of central venous catheter-related infection in the intensive care unit. Crit Care 14, 212 (2010).

77 UpToDate, I. Central venous catheter for hemodialysis, <http://www.uptodate.com/contents/image?imageKey=NEPH/13155> (2011).

78 Cesar, S., Garcia, A., Parada, E. \& Soriano, A. Cavernous sinus thrombosis due to invasive community-associated methicillin-resistant Staphylococcus aureus infection. Enferm Infecc Microbiol Clin 28, 755-756 (2010).

79 Keller, J. E., Hindman, J. W., Mehall, J. R. \& Smith, S. D. Enoxaparin inhibits fibrin sheath formation and decreases central venous catheter colonization following bacteremic challenge. Crit Care Med 34, 1450-1455 (2006).

80 Mehall, J. R., Saltzman, D. A., Jackson, R. J. \& Smith, S. D. Catheter materials affect the incidence of late blood-borne catheter infection. Surg Infect (Larchmt) 2, 225-229; discussion 229-230 (2001).

81 Mehall, J. R., Saltzman, D. A., Jackson, R. J. \& Smith, S. D. Fibrin sheath enhances central venous catheter infection. Crit Care Med 30, 908-912 (2002).

82 Blot, S. I. et al. Clinical and economic outcomes in critically ill patients with nosocomial catheter-related bloodstream infections. Clin Infect Dis 41, 1591-1598 (2005).

83 O'Grady N, P. et al. Guidelines for the prevention of intravascular catheter-related infections. Am J Infect Control 30, 476-489 (2002).

84 Medicaid program; payment adjustment for provider-preventable conditions including health care-acquired conditions. Final rule. Fed Regist 76, 32816-32838 (2011).

85 Bradshaw, J. H. \& Puntis, J. W. Taurolidine and catheter-related bloodstream infection: a systematic review of the literature. J Pediatr Gastroenterol Nutr 47, 179-186 (2008). 
86 Snaterse, M., Ruger, W., Scholte Op Reimer, W. J. \& Lucas, C. Antibiotic-based catheter lock solutions for prevention of catheter-related bloodstream infection: a systematic review of randomised controlled trials. J Hosp Infect 75, 1-11 (2010).

87 Cicalini, S., Palmieri, F. \& Petrosillo, N. Clinical review: new technologies for prevention of intravascular catheter-related infections. Crit Care 8, 157-162 (2004).

88 Ramritu, P. et al. A systematic review comparing the relative effectiveness of antimicrobialcoated catheters in intensive care units. Am J Infect Control 36, 104-117 (2008).

89 Pai, M. P., Pendland, S. L. \& Danziger, L. H. Antimicrobial-coated/bonded and -impregnated intravascular catheters. Ann Pharmacother 35, 1255-1263 (2001).

90 Halton, K. \& Graves, N. Economic evaluation and catheter-related bloodstream infections. Emerg Infect Dis 13, 815-823 (2007).

91 Halton, K. A., Cook, D. A., Whitby, M., Paterson, D. L. \& Graves, N. Cost effectiveness of antimicrobial catheters in the intensive care unit: addressing uncertainty in the decision. Crit Care 13, R35 (2009).

92 Hockenhull, J. C. et al. The clinical effectiveness and cost-effectiveness of central venous catheters treated with anti-infective agents in preventing bloodstream infections: a systematic review and economic evaluation. Health Technol Assess 12, iii-iv, xi-xii, 1-154 (2008).

93 Baker, C., Pradhan, A., Pakstis, L., Pochan, D. J. \& Shah, S. I. Synthesis and antibacterial properties of silver nanoparticles. J Nanosci Nanotechnol 5, 244-249 (2005).

94 Lok, C. N. et al. Silver nanoparticles: partial oxidation and antibacterial activities. J Biol Inorg Chem 12, 527-534 (2007).

95 Tseng, K. H. \& Liao, C. Y. Production of silver ions from colloidal silver by nanoparticle iontophoresis system. J Nanosci Nanotechnol 11, 1991-1995 (2011).

96 He, T. \& Chan, V. Covalent layer-by-layer assembly of polyethyleneimine multilayer for antibacterial applications. J Biomed Mater Res A 95, 454-464 (2010).

97 Maki, D. G. In vitro studies of a novel antimicrobial luer-activated needleless connector for prevention of catheter-related bloodstream infection. Clin Infect Dis 50, 1580-1587 (2010).

98 Roe, D., Karandikar, B., Bonn-Savage, N., Gibbins, B. \& Roullet, J. B. Antimicrobial surface functionalization of plastic catheters by silver nanoparticles. I Antimicrob Chemother 61, 869-876 (2008).

99 Yao, Y., Ohko, Y., Sekiguchi, Y., Fujishima, A. \& Kubota, Y. Self-sterilization using silicone catheters coated with $\mathrm{Ag}$ and $\mathrm{TiO} 2$ nanocomposite thin film. J Biomed Mater Res B Appl Biomater 85, 453-460 (2008).

100 Hsu, S.-h., Tseng, H.-J. \& Lin, Y.-C. The biocompatibility and antibacterial properties of waterborne polyurethane-silver nanocomposites. Biomaterials 31, 6796-6808 (2010).

101 Hung, H.-S. \& Hsu, S.-H. Biological performances of poly(ether)urethane-silver nanocomposites. Nanotechnology 18, 475101 (2007).

102 Ningbo TianHou Import and Export Co., L. Central venous catheters, $<$ http://tenhoo.en.hisupplier.com/product-283237-Central-Venous-Catheter.html> (2011).

103 Chin-Quee, S. L. et al. Endothelial cell recovery, acute thrombogenicity, and monocyte adhesion and activation on fluorinated copolymer and phosphorylcholine polymer stent coatings. Biomaterials 31, 648-657 (2010).

104 McClung, W. G., Babcock, D. E. \& Brash, J. L. Fibrinolytic properties of lysine-derivatized polyethylene in contact with flowing whole blood (Chandler loop model). J Biomed Mater Res A 81, 644-651 (2007).

105 Mutch, N. J. et al. The use of the Chandler loop to examine the interaction potential of NXY059 on the thrombolytic properties of $\mathrm{rtPA}$ on human thrombi in vitro. Br J Pharmacol 153, 124-131 (2008).

106 Paul, A. et al. Aptamers influence the hemostatic system by activating the intrinsic coagulation pathway in an in vitro Chandler-Loop model. Clin Appl Thromb Hemost 16, 161-169 (2010). 
107 Waterhouse, A. et al. The immobilization of recombinant human tropoelastin on metals using a plasma-activated coating to improve the biocompatibility of coronary stents. Biomaterials 31, 8332-8340 (2010).

108 Nilsson, B., Ekdahl, K. N., Mollnes, T. E. \& Lambris, J. D. The role of complement in biomaterial-induced inflammation. Mol Immunol 44, 82-94 (2007).

109 Hudacko, A., Sievert, A. \& Sistino, J. Gaseous microemboli in a pediatric bypass circuit with an unprimed venous line: an in vitro study. J Extra Corpor Technol 41, 166-171 (2009).

110 Melchior, R. W., Rosenthal, T. \& Glatz, A. C. An in vitro comparison of the ability of three commonly used pediatric cardiopulmonary bypass circuits to filter gaseous microemboli. Perfusion 25, 255-263; discussion 265-256 (2010).

111 Myers, G. J., Voorhees, C., Eke, B. \& Johnstone, R. The effect of Diprivan (propofol) on phosphorylcholine surfaces during cardiopulmonary bypass--an in vitro investigation. Perfusion 24, 349-355 (2009).

112 Thiara, A. S., Eggereide, V., Pedersen, T., Lindberg, H. \& Fiane, A. E. In vitro and in vivo evaluation of Dideco's paediatric cardiopulmonary circuit for neonates weighing less than five kilograms. Perfusion 25, 229-235 (2010).

113 Hemker, H. C., Giesen, P. L., Ramjee, M., Wagenvoord, R. \& Beguin, S. The thrombogram: monitoring thrombin generation in platelet-rich plasma. Thromb Haemost 83, 589-591 (2000).

114 Stevens, K. N. et al. The relationship between the antimicrobial effect of catheter coatings containing silver nanoparticles and the coagulation of contacting blood. Biomaterials 30, 3682-3690 (2009).

115 Aldenhoff, Y. B., Hanssen, J. H., Knetsch, M. L. \& Koole, L. H. Thrombus formation at the surface of guide-wire models: effects of heparin-releasing or heparin-exposing surface coatings. J Vasc Interv Radiol 18, 419-425 (2007).

116 Aldenhoff, Y. B. et al. Coils and tubes releasing heparin. Studies on a new vascular graft prototype. Biomaterials 25, 3125-3133 (2004).

117 Rijnders, B. J., Van Wijngaerden, E. \& Peetermans, W. E. Catheter-tip colonization as a surrogate end point in clinical studies on catheter-related bloodstream infection: how strong is the evidence? Clin Infect Dis 35, 1053-1058 (2002).

118 Maki, D. G., Weise, C. E. \& Sarafin, H. W. A semiquantitative culture method for identifying intravenous-catheter-related infection. N Engl J Med 296, 1305-1309 (1977).

119 Safdar, N., Fine, J. P. \& Maki, D. G. Meta-analysis: methods for diagnosing intravascular device-related bloodstream infection. Ann Intern Med 142, 451-466 (2005).

120 Slobbe, L., El Barzouhi, A., Boersma, E. \& Rijnders, B. J. Comparison of the roll plate method to the sonication method to diagnose catheter colonization and bacteremia in patients with long-term tunnelled catheters: a randomized prospective study. $J$ Clin Microbiol 47, 885-888 (2009). 
General introduction 


\section{2}

Bioengineering of improved biomaterials coatings for extracorporeal circulation requires extended observation of blood-biomaterial interaction under flow

Stevens $\mathrm{KN}^{1}$, Aldenhoff $\mathrm{YB}^{2}$, van der Veen $\mathrm{FH}^{1}$, Maessen $\mathrm{JG}^{1}$, Koole $\mathrm{LH}^{2}$.

1. Department of Cardiothoracic Surgery, Maastricht University Medical Centre, Maastricht, The Netherlands.

2. Centre for Biomaterials Research, University of Maastricht, Maastricht, The Netherlands.

J Biomed Biotechnol. 2007;2007(10):29464. 


\section{Abstract}

Extended use of cardiopulmonary bypass (CPB) systems in cardiac surgery is often problematic, due to the formation of thrombotic emboli and infection. Part of these problems relates to imperfect blood compatibility of the artificial surfaces within the CPB circuits. The engineering of biomaterial surfaces with genuine long-term blood compatibility is far from trivial. Rational selection of different materials or surfaces must rely on long-term blood compatibility, which is essentially virgin territory in biomaterials science. For example, most experiments with the well-known Chandler loop model system for evaluation of blood-biomaterial interactions under flow conditions have been described for a maximum duration of $2 \mathrm{~h}$ only. This study reports a systematic evaluation of two commercial CPB tubings, each with a blood compatible coating on the inner surface, and one uncoated control. The experiments comprised: (i), testing with whole human blood from 4 different donors under flow; (ii) measurement of the most essential blood parameters (platelet counts, markers of platelet activation, leukocyte activation and complement activation) at 5 different time points (0, 75, 150, 225 and $300 \mathrm{~min})$; (iii), analysis of the luminal surfaces with scanning electron microscopy (again at the same time points). Furthermore, the different materials were subjected to a thrombin generation test in vitro. The complete data set (three materials, four donors, five time points) indicated differences in hemocompatibility of the tubings. Furthermore, it appeared that discrimination between biomaterial coatings can be made only after several hours of blood-biomaterial contact. Platelet counting, determination of myeloperoxidase (a marker of inflammation), and scanning electron microscopy proved to be the most useful methods. These findings are believed to be of interest and relevance with respect to the bioengineering of extracorporeal devices that should function in contact with blood for extended time. 


\section{Introduction}

Cardiopulmonary bypass (CPB) technology represents one of the most striking examples of progress in biomedical engineering. Procedures in cardiac surgery that rely on $\mathrm{CPB}$ can nowadays be regarded as safe, i.e., they are associated with a low incidence of mortality ${ }^{1,2,3}$. These developments resulted, to a significant extent, from improvements in the polymeric biomaterials that constitute the inner surface of $\mathrm{CPB}$ circuits. Within $\mathrm{CPB}$ circuits, there is extensive blood contact with the tubing, the pump, and -particularly- the oxygen/carbon dioxide exchange membrane. It is well known that cellular components of the blood (particularly leukocytes and platelets) may become activated, and that four different but partially overlapping plasma protein cascades will go into operation (intrinsic and extrinsic clotting cascade, complement system, and fibrinolytic protein system) ${ }^{4}$. For more than 4 decades, heparin has been used as the standard "anticoagulant" to counterbalance these effects. Improvements resulted from the use of surface coatings exposing heparin at the blood-biomaterial interface. These coatings reduce coagulation, inflammation, complement activation and platelet activation ${ }^{1,4}$. More recently, $\mathrm{CPB}$ equipment has been coated with poly(2-methoxy-ethylacrylate) (PMEA), based on the hypothesis that this material leads to improved blood compatibility compared to uncoated surfaces. PMEA is cheap compared to heparin-exposing coatings, and was postulated to provide a useful alternative for patients with heparin-associated disorders ${ }^{5,6,7}$.

However, serious complications may arise if extracorporeal circulation has to be sustained, e.g. for several days. The most frequent problems stem from bacterial infection, hemolysis, thrombus formation within the circuit, and formation of circulating thrombotic emboli ${ }^{8,9,10}$. We became intrigued by these problems, since they relate to long-term hemocompatibility of polymeric materials, which is in fact unexplored territory in biomaterials science. Indeed, we noticed that the literature on blood compatibility of CPB circuits merely contains experimental data that correspond to short testing periods. For example, Weber et al. extensively studied hemocompatibility of 4 different biomaterials in a CPB model, but only up to $120 \mathrm{~min}^{11}$. We adhere to the idea that successful development of novel biomaterials or biomaterial coatings for $\mathrm{CPB}$ will depend on robust evaluation models in which the blood-biomaterial contact is maintained for several hours at least.

Herein we report a systematic methodological study in which two commercial surface-coated CPB tubings (heparin coated tubings and PMEA coated tubings) and one uncoated control were evaluated in contact with human whole blood under flow, for a period of 5 hours. We calculated that $5 \mathrm{~h}$ of experimentation implies a level of blood-biomaterial contact that corresponds to at least $9 \mathrm{~h}$ of operation in a typical CPB system (vide infra). 
Several assays were used to evaluate the blood (platelet counts and assays to determine hemolysis, platelet activation, leukocyte activation and activation of the complement system). Scanning electron microscopy (SEM) was used to study deposition of blood components at the surface of the tubings. A full set of data was acquired for the three different materials, four donors and five time points $(0,75,150,225$ and $300 \mathrm{~min})$. The three materials showed clear differences, in general pointing towards an inferior hemocompatibility of the PMEA coating. Moreover, two points with respect to bioengineering of improved coatings for long-term CPB emerged: (i) since most of the differences between the three surfaces did not become apparent during the first $2 \mathrm{~h}$ of experimentation, long-term (e.g. 5 h) testing of blood-biomaterial interactions under flow is required; (ii) preferably, parallel tests with blood from several different donors should be performed, since the results from several assays appeared to be clearly donor-dependent.

\section{Materials \& methods}

\section{Materials}

Poly(vinylchloride) (PVC) tubings with a coating of PMEA were a generous gift of Terumo Europe NV (Leuven, Belgium). The internal diameter of the tubings was $0.476 \mathrm{~cm}$. The same company also provided the uncoated tubings with identical internal diameter, which were used as controls. Tubings with a coating of heparin were obtained from Maquet Cardiopulmonary AG (Hirrlingen, Germany). The internal diameter was also $0.476 \mathrm{~cm}$. All tubings were received in a sterile package and cut to length $(42.5 \mathrm{~cm})$ immediately prior to the experiments. Lepirudin (Refludan $\left.{ }^{\circledR}\right)$ was purchased from Pharmion (Windsor Berkshire, United Kingdom). Bovine serum albumin (BSA), Nacitrate, ethylenediaminetetraacetic acid (EDTA) and Zymosan A were from Sigma-Aldrich Chemie B.V. (Zwijndrecht, The Netherlands). 4-(2-hydroxyethyl)1-piperazineethanesulfonic acid (HEPES), $\mathrm{NaCl}, \mathrm{KCl}$ and Glutaraldehyde 25\% were from Acros Organics (Geel, Belgium). Na2HPO4 and KH2PO4 were from Janssen Chimica (Beerse, Belgium). Ethanol 100\% was from Merck KGaA (Darmstadt, Germany). The chromogenic substrate S2238 was synthesized according to Rijkers et al. ${ }^{12}$. The following solutions were prepared: a lepirudin stock solution (lepirudin $200 \mu \mathrm{g} / \mathrm{mL}, \mathrm{NaCl} 9 \mathrm{~g} / \mathrm{L}$ ), a HEPES/EDTA stock solution (HEPES $100 \mathrm{mM}$, EDTA $40 \mathrm{mM}, \mathrm{pH}$ 7.4), a phosphate buffered saline (PBS) solution $(\mathrm{NaCl} 8 \mathrm{~g} / \mathrm{L}, \mathrm{KCl} 0.2 \mathrm{~g} / \mathrm{L}, \mathrm{Na} 2 \mathrm{HPO} 41.44 \mathrm{~g} / \mathrm{L}, \mathrm{KH} 2 \mathrm{PO} 40.24$ $\mathrm{g} / \mathrm{L}, \mathrm{pH}$ 7.4), a $\mathrm{CaCl} 2$ stock solution $(0.5 \mathrm{M} \mathrm{CaCl} 2)$, a Na-citrate stock solution (Na-citrate $0.13 \mathrm{M}$ ), an S2238 stock solution (S2238 $2 \mathrm{mM}$ ) and a stop buffer ( NaCl 140 mM, HEPES 20 mM, EDTA 20 mM, BSA 1 mg/mL, S2238 stock 
solution 1/10, $\mathrm{pH} 7.5)$. Citrate-theophylline-adenosine-dipyridamole (CTAD) stock solution (BD Vacutainer ${ }^{\circledR}$ CTAD Tubes) was a product from Becton Dickinson (Alphen aan den Rijn, The Netherlands). The enzyme-linked immunosorbent assay (ELISA) for $\beta$-thromboglobulin $(\beta-T G)$ (Asserachrom ${ }^{\circledR}$ $\beta-T G$ ) was purchased from Diagnostica Stago (Asnières sur Seine, France) and ELISA kits for terminal complement complex (TCC) and myeloperoxidase (MPO) were from Hycult biotechnology B.V. (Uden, The Netherlands).

\section{Equipment}

Experiments were performed on a modified Chandler loop system, which was equipped with a broad wheel with a diameter of $13 \mathrm{~cm}^{13}$. On this wheel, 12 tubes could be rotated simultaneously. The rotating speed was set at 32 per minute. The rotating wheel and the mounted tubes were immersed in a water bath that was kept at $37^{\circ} \mathrm{C}$ throughout the entire experiment. The Chandler loop device was made by the mechanical workshop of the Instrument Development Engineering \& Evaluation of the University Maastricht. Centrifugation was performed with an Eppendorf Centrifuge 5417C (Eppendorf, Hamburg, Germany). Platelets were counted on an automatic cell counter (Coulter ${ }^{\circledR}$ AC-T diff, Beckman Coulter, Miami, United States). The absorbance of plasma free hemoglobin $(\mathrm{Hb})$ was determined on a spectrophotometer (Multiskan ${ }^{\circledR}$ Spectrum Microplate Spectrophotometer, Thermo Labsystems, Vantaa, Finland). Microtiter plates were heated on a plate warmer (Single Micro-Hywel ${ }^{\mathrm{TM}}$, Chromogenix, Milano, Italy). For both the ELISA assays and the thrombin generation time assay, the absorbances of the microtiter plates were determined spectrophotometrically on a microplate reader (ELx808 ${ }^{\mathrm{TM}}$ Absorbance Microplate Reader, BioTek Instruments, Inc., Vermont, USA). Samples for SEM were coated with gold on a sputter coater (Sputter coater 108 auto/SE, Cressington Scientific Instruments Ltd., Watford, United Kingdom) and then analyzed with a scanning electron microscope (Philips XL30 Scanning Electron Microscope, Philips, Eindhoven, The Netherlands).

\section{Experiments under flow conditions: the Chandler loop model}

This study was approved by the Ethical Committee of the University of Maastricht. Four healthy male blood donors (further indicated by their initials as WW, KS, SB and JB) aged between 20 and 25 years old were included in this study. They were all non-smokers and did not take any haemostasis-influencing medicines at least 10 days before the experiment. Each donor visited our laboratories twice and donated blood for two different experiments; there were at least 7 days between the two visits. 
Hemocompatibility analysis by platelet counting and assessment of hemolysis (performed after the first visit of each donor)

Blood was withdrawn by venipuncture and immediately anticoagulated with lepirudin stock solution (1 part lepirudin stock solution and 9 parts whole blood), following recommendations made by Kopp et al. ${ }^{14}$. Directly after blood collection, $1.35 \mathrm{~mL}$ of blood was sampled and processed as described further, to obtain baseline values. Next, three different tubes (one heparin coated tube, one PMEA coated tube and one uncoated control tube) were each filled with 6.7 $\mathrm{mL}$ whole blood, which corresponds to a degree of filling of $88 \%$. The tubes were then closed end-to-end using silicon sleeves, mounted on the rotating wheel and rotated in a water bath at $37^{\circ} \mathrm{C}$ and $32 \mathrm{rpm}$.

From each tube, $1.35 \mathrm{~mL}$ blood was withdrawn after 75, 150, 225 and 300 min of incubation; note that in the tubes the degree of filling gradually dropped from $88 \%$, via $70 \%$ and $53 \%$ to $35 \%$. Immediately after withdrawal from the tube, each blood sample was mixed with $150 \mu \mathrm{L}$ HEPES/EDTA stock solution. One third of this mixture $(500 \mu \mathrm{L})$ was used for platelet counting; these counts were performed in triplicate.

The other part of the HEPES/EDTA-mixed blood sample $(1 \mathrm{~mL})$ was processed for assessment of hemolysis. The percentage of plasma free $\mathrm{Hb}$ was used as an indicator for hemolysis. $25 \mu \mathrm{L}$ of HEPES/EDTA-mixed blood sample was diluted 40 times with $975 \mu \mathrm{L}$ deionized water, to achieve total hemolysis. The other $975 \mu \mathrm{L}$ of HEPES/EDTA-mixed blood sample was kept undiluted. Both the diluted and undiluted parts were centrifuged (3220 g, $20 \mathrm{~min}, 4^{\circ} \mathrm{C}$ ) to obtain plasma. Subsequently, the absorbance was measured at three wavelengths $(560,576$ and $592 \mathrm{~nm}$ ) in plasma of both the diluted and undiluted parts of the HEPES/EDTA-mixed blood sample. The percentage of plasma free $\mathrm{Hb}$ was then calculated for each blood sample according to the procedure of Cripps ${ }^{15}$.

Hemocompatibility analysis by quantification of blood activation markers via ELISA and SEM of the tube inner surfaces (performed after the second visit of each donor)

Blood was withdrawn by venipuncture and immediately anticoagulated with lepirudin stock solution (1 part lepirudin stock solution and 9 parts whole blood), following recommendations made by Kopp et al. ${ }^{14}$. Immediately after blood collection, a $4.5 \mathrm{~mL}$ and $1.8 \mathrm{~mL}$ blood sample were isolated and processed as described further, to obtain baseline values. Next, twelve tubes (four heparin coated tubes, four PMEA coated tubes and four uncoated control tubes) were each filled with $6.7 \mathrm{~mL}$ whole blood, which corresponds to a degree of filling of $88 \%$. The tubes were then closed end-to-end using silicon sleeves, mounted on the rotating wheel and rotated in a water bath at $37^{\circ} \mathrm{C}$ and $32 \mathrm{rpm}$. 
After 75, 150, 225 and 300 min of incubation, each time three tubes (one heparin coated tube, one PMEA coated tube and an uncoated control tube) were removed from the rotating wheel. Two blood samples were isolated from each tube: $4.5 \mathrm{~mL}$ blood was withdrawn and immediately mixed with $0.5 \mathrm{~mL}$ CTAD stock solution, and $1.8 \mathrm{~mL}$ of blood was withdrawn and immediately mixed with $0.2 \mathrm{~mL}$ HEPES/EDTA stock solution. Both the CTAD-mixed and HEPES/EDTA-mixed blood were incubated on ice for 15 minutes. Then, plasma was isolated by two subsequent centrifugation steps (2550 g, $20 \mathrm{~min}$, $4{ }^{\circ} \mathrm{C}$ ). The plasma was aliquoted and stored at $-80{ }^{\circ} \mathrm{C}$ until further analysis. ELISA was used to evaluate activation of leukocytes, complement and platelets. As a marker for leukocyte activation the levels of MPO were quantified in HEPES/EDTA-stabilized plasma. The concentration of $\beta$-TG in CTADstabilized plasma served as a marker for platelet activation. Complement activation was investigated by measuring plasma concentrations of TCC in HEPES/EDTA-stabilized plasma. Zymosan A activated whole blood was used as a positive control for complement activation.

Following isolation of the blood samples, each tube was prepared for SEM analysis. Non-adherent blood components were washed away by rinsing the tubes extensively with 25 volumes of PBS solution. Next, adherent blood components were fixed by incubating the tubes overnight in $2.5 \%$ glutaraldehyde at $2-8{ }^{\circ} \mathrm{C}$. Fixed samples were dehydrated by immersion in an ethanol series (50, 70, 80, 95 and 100\% ethanol). Following dehydration, the samples were air dried. For each time point, three pieces of air dried tubing with a length of $1 \mathrm{~cm}$ were cut out. The pieces were then cut lengthwise for analysis of the inner surface. This was done for all donors. Finally, the samples were sputter coated with gold and imaged with a scanning electron microscope.

\section{Experiments under static conditions: thrombin generation time assay}

This assay was carried out as described previously ${ }^{16,17,18,19}$. Briefly, blood was withdrawn by venipuncture from a healthy, non-smoking male blood donor who did not take any haemostasis-influencing medicines at least 10 days before the experiment. The blood was immediately anticoagulated with citrate stock solution (1 part citrate stock solution and 9 parts whole blood) and kept at $37{ }^{\circ} \mathrm{C}$ until the start of the experiment. Uncoated, heparin coated and PMEA coated PVC tubes were cut to a length of $5.5 \mathrm{~cm}$. These pieces were closed at one end with a tube clamp. At the start of the experiment blood was recalcified with $40 \mu \mathrm{L} \mathrm{CaCl} 2$ stock solution per mL blood. Subsequently, $750 \mu \mathrm{L}$ of blood was added to each tube sample and the tube samples were incubated at $37{ }^{\circ} \mathrm{C}$ under static conditions. After 5 minutes of incubation, $17.5 \mu \mathrm{L}$ of blood was taken from each tube sample and mixed with $282.5 \mu \mathrm{L}$ stop buffer. Sampling was done every 5 minutes until 24 minutes of incubation, from then samples were 
taken every 2 minutes. Blood samples were kept on ice until further handling. At the end of the experiment, the blood samples were centrifuged at $10621 \mathrm{~g}$ for 5 minutes. Next, $200 \mu \mathrm{L}$ plasma of each sample was loaded onto a microtiter plate that was kept on ice. After loading, the microtiter plate was heated for 5 min at $37{ }^{\circ} \mathrm{C}$. Finally, thrombin concentrations were measured using absorption spectrophotometry at $405 \mathrm{~nm}$.

\section{Statistics}

Statistical analysis was performed using Mann-Whitney U analysis for between group comparisons and the Wilcoxon test for paired observations for comparison within groups. A p-value less than 0.05 (two-tailed) was considered significant.

\section{Results \& discussion}

\section{Analysis of the blood samples}

\section{Platelet counts}

Figure 1 compiles the results on the platelet count experiments; note that data referring to the three different biomaterials, based on blood from four different donors, and measured at five time points are combined. This format is used consistently throughout this article. At the start of the experiment, the donors had platelet counts between 75,000 and 180,000 per $\mu \mathrm{L}$, which is within the normal range. Some increased spreading is noted, especially in the counts that were measured after 225 or $300 \mathrm{~min}$. The heparin coated specimens and the uncoated controls show invariant platelet counts, as a function of time. The PMEA coating, on the other hand, induces a decrease in the concentration of circulating platelets. For donor JB, this effect can be noticed already after $75 \mathrm{~min}$, and the decrease goes on during the entire experiment. For the other donors, platelet counts start to decline only after 150 $\mathrm{min}$ (KS and SB), or after $225 \mathrm{~min}(\mathrm{WW})$. The largest drop in platelet count was found with the blood from donor JB in the PMEA-coated tube; the concentration of circulating platelets decreased by almost $60 \%$, from ca. 140,000 per $\mu \mathrm{L}$ to ca. 60,000 per $\mu \mathrm{L}$. At the end of the experiment the concentration of circulating platelets was significantly lower in the PMEA coated tubes compared to the heparin coated tubes $(79,917 \pm 6,304$ platelets $/ \mu \mathrm{L}$ blood for PMEA coated tubes vs 135,333 $\pm 10,623$ platelets/ $\mu \mathrm{L}$ blood for heparin coated tubes (mean \pm sem), $\mathrm{p}=0.001)$ and the uncoated controls $(79,917 \pm 6,304$ platelets / $\mu \mathrm{L}$ blood for PMEA coated tubes vs $133,833 \pm 10,650$ platelets $/ \mu \mathrm{L}$ 


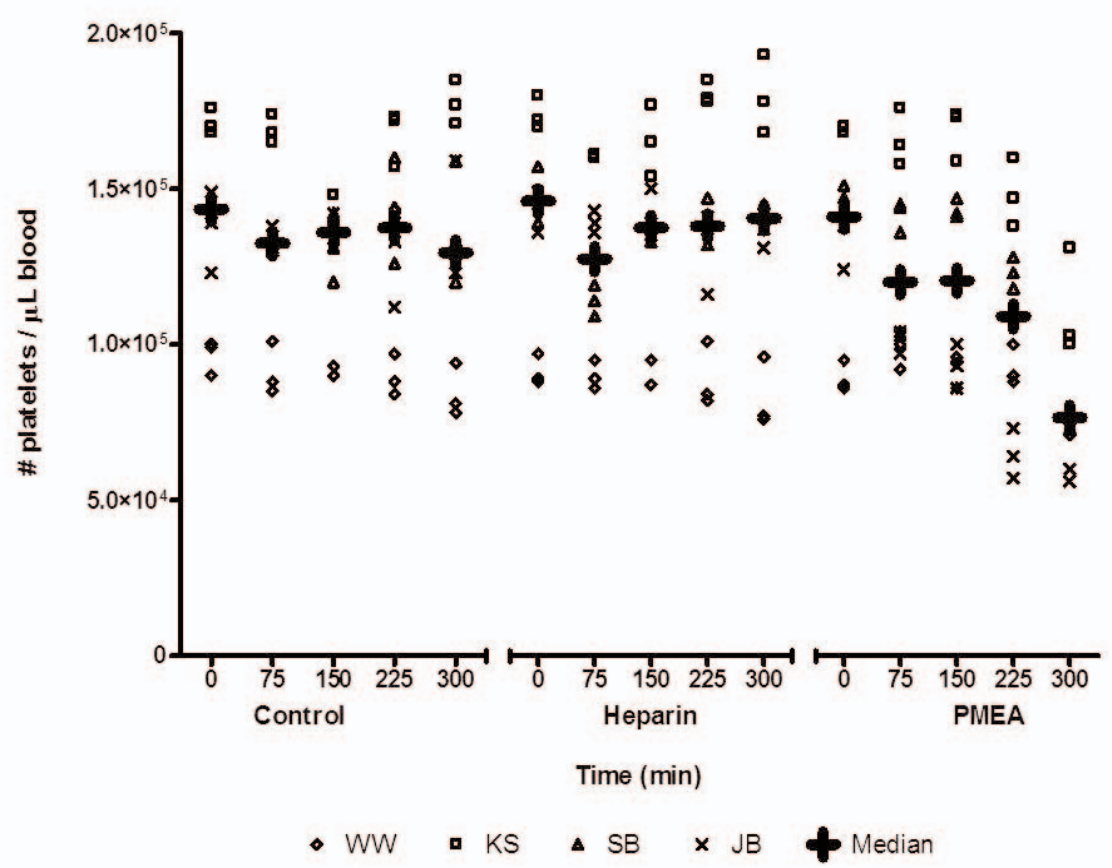

Figure 1. Platelet counts for the individual donors. Data are shown at five time points for each tubing surface tested. Measurements were performed in triplicate. Statistical differences between the platelet counts in the different tubes were found after $300 \mathrm{~min}$, as indicated.

blood for uncoated controls (mean \pm sem), $\mathrm{p}=0.001)$. This indicated that the PMEA coating has a propensity to activate contacting platelets. This is, most probably not a direct effect, but an effect of plasma proteins, adsorbed onto the PMEA surface. Activated platelets are known to adhere to adsorbed fibrinogen, von Willebrand factor, vitronectin and fibronectin ${ }^{20}$. Consequently this results in lower platelet counts. In addition, activated platelets adhere to each other via fibrinogen bridges, anchored by GPIIb/IIIa receptors ${ }^{21}$. Activated platelets also adhere to circulating leukocytes ${ }^{22,23}$. Some of the surface-attached platelets partially detach, leaving adherent membrane fragments ${ }^{24}$. Other platelets are damaged by shear forces ${ }^{25}$. Recently, platelet counts were also used in a clinical study to evaluate hemocompatibility of heparin and PMEA coated CPB circuits. Kutay and co-workers reported a more significant depletion of circulating platelets in PMEA coated circuits compared to heparin coated circuits ${ }^{26}$.

\section{$\underline{\text { Hemolysis }}$}

Figure 2 shows the data set resulting from our concentration measurements of extracellular (free) Hb. Rupture of erythrocytes (hemolysis), e.g. due to collisions with the lumen of the tubes, leads to release of $\mathrm{Hb}$ into the plasma. The vertical axis in Figure 2 depicts the ratio free $\mathrm{Hb}$ : total $\mathrm{Hb}$, 
expressed as a percentage. Clearly, hemolysis is very low, i.e. most of the erythrocytes remained intact in all cases. Even in the most extreme situation (donor JB, PMEA coating, 300 min circulation), only 1 out of every 400 molecules $\mathrm{Hb}$ is free. Despite the occurrence of little hemolysis, it is clear that the level of free $\mathrm{Hb}$ in the plasma increases with circulation time, which was expected. The three coatings do not perform differently in this respect. No significant differences in the levels of plasma free $\mathrm{Hb}$ were found between the different tubes. The hemolysis assay applied in this study was based on the Cripps method to measure levels of plasma free $\mathrm{Hb}^{15}$. In a study by Malinauskas $\mathrm{RA}^{27}$, this method was shown to be more precise and accurate than the chemical addition methods to measure levels of plasma free $\mathrm{Hb}$.

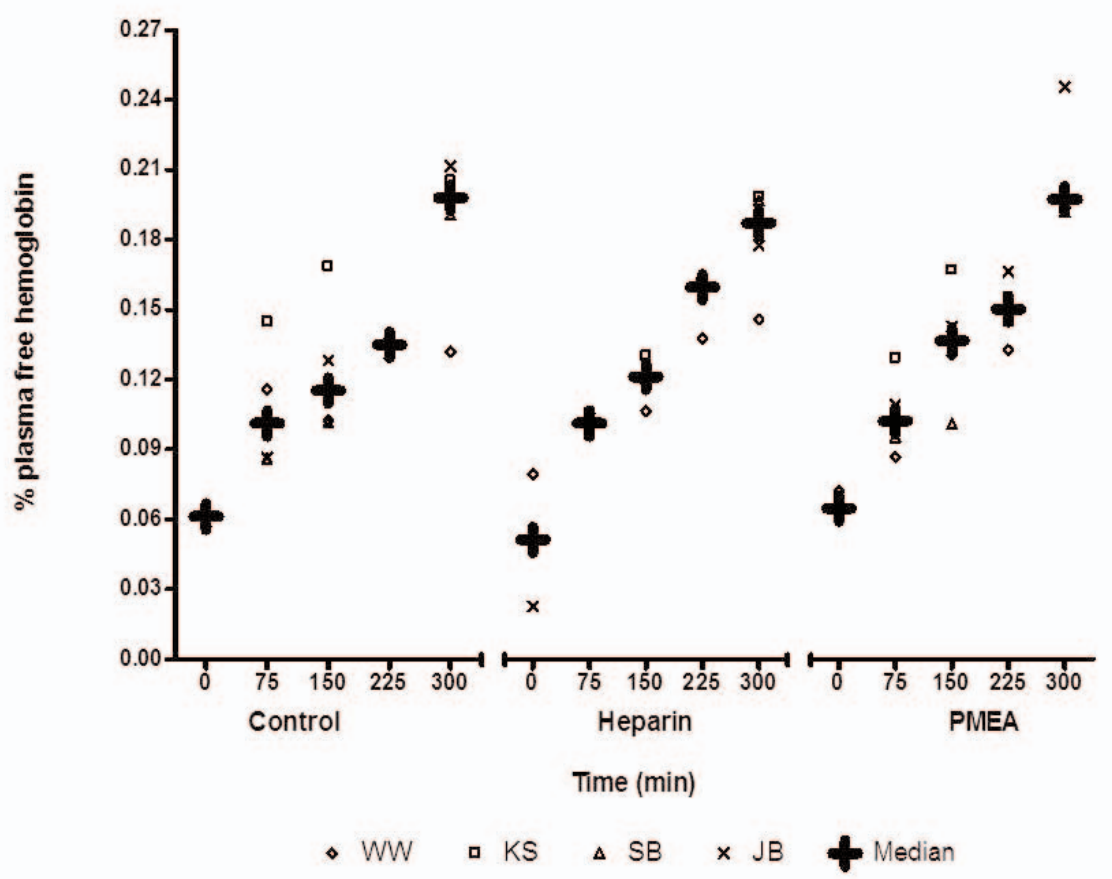

Figure 2. Percentage of plasma free $\mathrm{Hb}$ for the individual donors. Data are shown at five time points for each tubing surface tested.

Activation of platelets: $\beta$-thromboglobulin

Figure 3 provides an overview of the concentration measurements of $\beta$-TG, which is a soluble marker for platelet activation ${ }^{28}$. Activated platelets release $\beta$-TG from their $\alpha$-granules. 


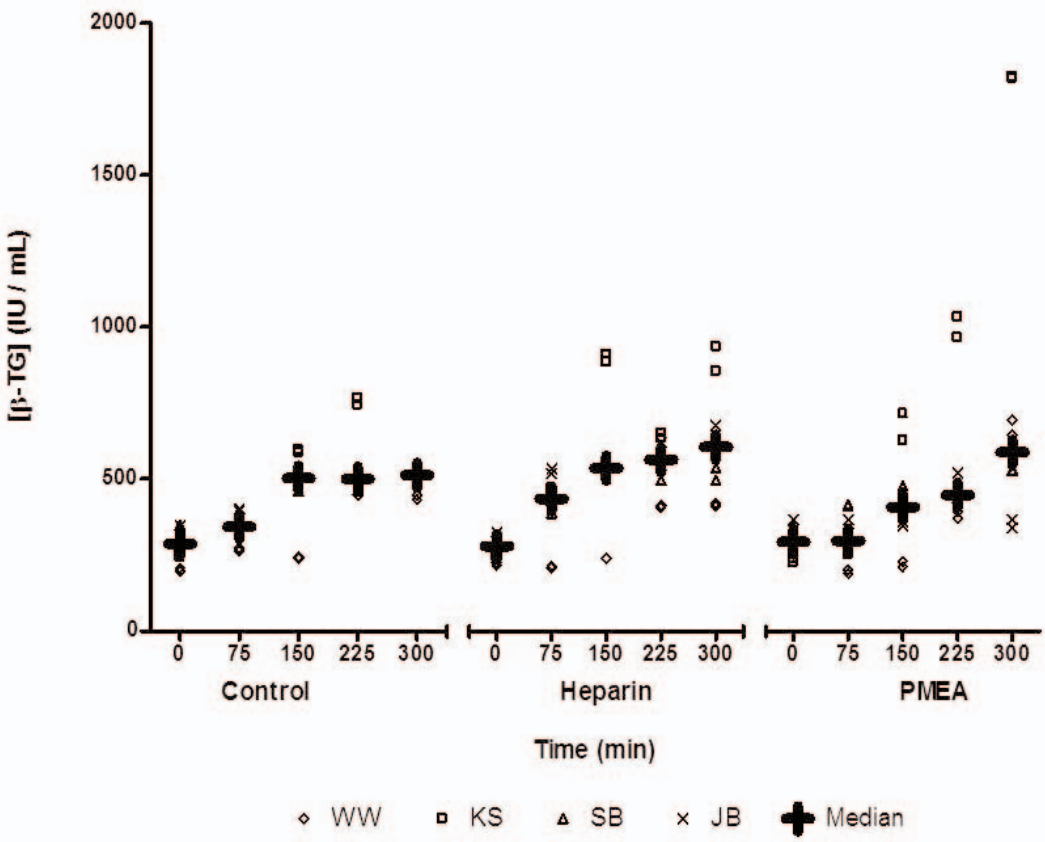

Figure 3. Concentrations of $\beta$-thromboglobulin for the individual donors. Data are shown at five time points for each tubing surface tested. Measurements were performed in duplicate.

The $\beta$-TG concentration vs. time profiles show an increasing trend, revealing that platelet activation progresses with time. With the blood of the donors WW and SB, this is found consistently for all coatings. The blood of donor JB follows this pattern, except in contact with PMEA: the concentration of $\beta$-TG then remains virtually unchanged. This is remarkable, since the platelet counts for this donor and this coating were found to decrease sharply (vide supra, Figure 1). Most likely, platelets of this donor adhere to PMEA without subsequent release of the granular contents. Donor KS is slightly aberrant. The levels of $\beta$-TG are relatively high, throughout all experiments, but it must be kept in mind that this donor also had the highest platelet counts (vide supra, Figure 1). In contact with the PMEA coating, a steep increase of the concentration of $\beta$-TG is found, after $150 \mathrm{~min}$ of incubation. However, there were no significant differences in platelet activation levels between uncoated, heparin coated and PMEA coated tubes. This indicates that immobilization of heparin or coating with PMEA do not help to reduce platelet activation within blood that contacts a PVC surface.

Activation of leukocytes: myeloperoxidase

During ECC, contact between the blood and artificial surfaces is known to induce an inflammatory response characterized by the activation of various 
leukocyte cell types ${ }^{22,29}$. Of the various leukocytes, neutrophils are the most abundant; they play a central role in the inflammatory response to $\mathrm{CPB}{ }^{27}$. MPO is a glycoprotein abundantly present in the primary granules of neutrophils; activated neutrophils release MPO by degranulation ${ }^{22,30,31}$.

Figure 4 presents the data of the concentration measurements of MPO. A clear pattern emerging from the data is that for every donor the concentration of MPO increases continuously. The rise in MPO levels was the highest in PMEA coated tubes. This resulted in significantly higher levels of MPO compared to heparin coated tubes, at time points 225 minutes $(163 \pm 11.6$ $\mathrm{ng} / \mathrm{mL}$ for PMEA coated tubes vs $131.5 \pm 4.8 \mathrm{ng} / \mathrm{mL}$ for heparin coated tubes (mean \pm sem), $\mathrm{p}=0.01)$ and 300 minutes $(222.3 \pm 11.5 \mathrm{ng} / \mathrm{mL}$ for PMEA coated tubes vs $157.7 \pm 3.6 \mathrm{ng} / \mathrm{mL}$ for heparin coated tubes (mean $\pm \mathrm{sem}), \mathrm{p}=$ $0.001)$, and uncoated controls, at time point 300 minutes $(222.3 \pm 11.5 \mathrm{ng} / \mathrm{mL}$ for PMEA coated tubes vs $176.8 \pm 9.8 \mathrm{ng} / \mathrm{mL}$ for uncoated controls (mean $\pm \mathrm{sem}$ ), $\mathrm{p}=0.01)$. These data indicate that the PMEA coating induces MPO release more abundantly as compared to both other surfaces. It is of interest to compare our data with a recent study of Lappegård et al., who also used the Chandler loop model to investigate neutrophil activation after blood-artificial surface contact ${ }^{22,29}$. After $4 \mathrm{~h}$ of blood circulation they found significantly lower levels of MPO release in heparin coated tubes compared to uncoated controls. Lappegarrd et al., however, used a heparin coating based on covalently end point attached heparin ${ }^{29}$, while the heparin coating used in our experiments involved both covalent and ionic interactions of heparin with the surface.

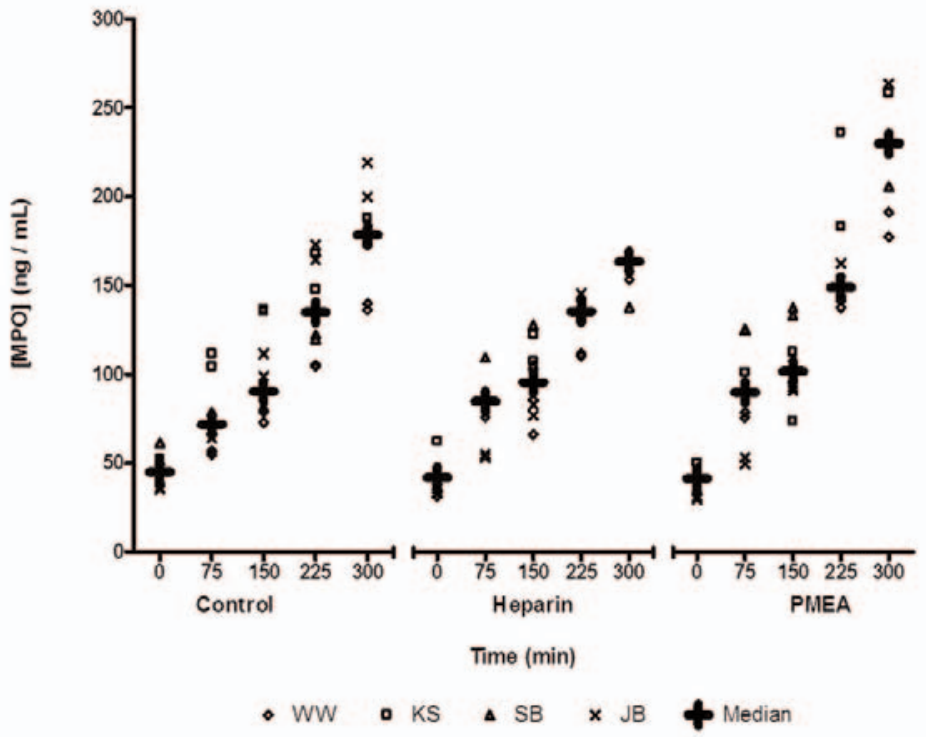

Figure 4. Concentrations of myeloperoxidase for the individual donors. Data are shown at five time points for each tubing surface tested. Measurements were performed in duplicate. Statistical differences between the MPO levels in the different tubes were found after 225 and $300 \mathrm{~min}$, as indicated. 
Our results appear to be in line with a recent clinical study by Kutay et al., who compared the hemocompatibility of PMEA and heparin coated CPB ${ }^{26}$. MPO levels at the end of CPB were significantly higher in the PMEA coated circuits compared to the heparin coated circuits. Besides MPO, plasma levels of interleukin-8, a pro-inflammatory cytokine, were also quantified by ELISA. In the three different tubes, plasma levels of interleukin- 8 remained undetectable until $225 \mathrm{~min}$ of incubation. At the end of the experiment, interleukin-8 generation did not differ significantly between the three tubes and plasma levels never exceeded $90 \mathrm{pg} / \mathrm{mL}$ (data not shown). Apparently, $5 \mathrm{~h}$ of bloodbiomaterial contact in our model is not sufficient to use interleukin-8 as a discriminating marker for inflammatory responses.

\section{Activation of the complement system: terminal complement complex}

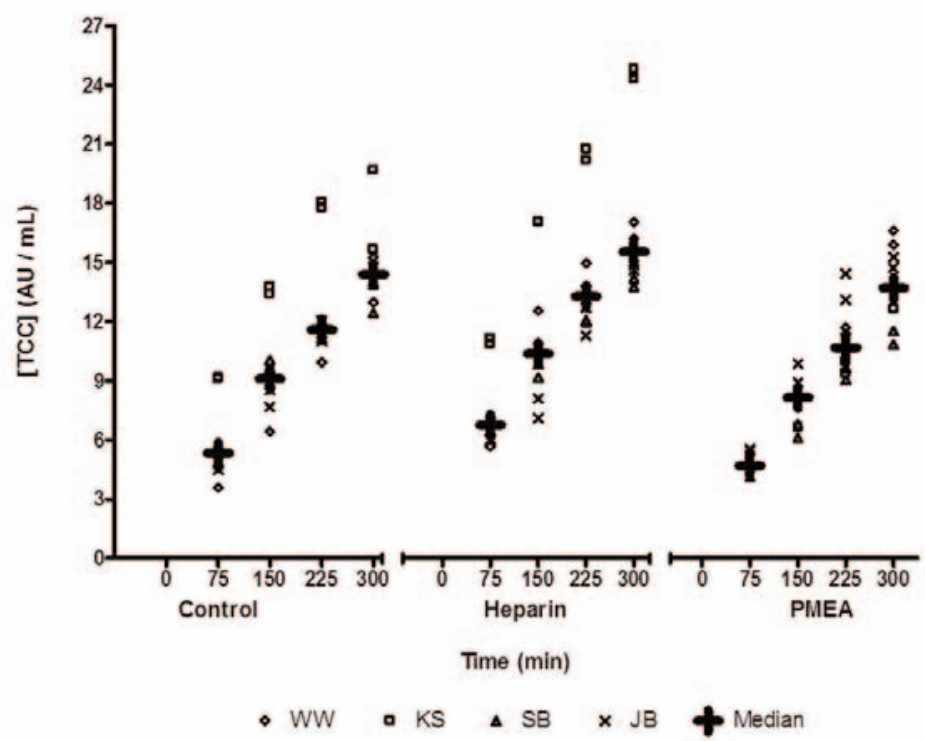

Figure 5. Concentrations of terminal complement complex for the individual donors. Data are shown at five time points for each tubing surface tested. Measurements were performed in duplicate. As can be seen, TCC was undetectable in all samples at time point 0h. Statistical differences between the TCC levels in the different tubes were found after 75, 150 and $225 \mathrm{~min}$, as indicated.

Complement activation has been studied extensively as a marker for hemocompatibility of artificial surfaces. Different components of the complement activation cascade can be used ${ }^{30}$. However, in a study by Gong et al. ${ }^{32}$, generation of complement activation products in the Chandler loop model displayed component-specific responsiveness to the size of the gas surface and the biomaterial surface. Of the complement activation markers evaluated, generation of TCC was least influenced by the size of the gas surface and mainly 
dependent on the biomaterial surface. This prompted us to use TCC as a complement activation marker in our study. Figure 5 depicts the data of the concentration measurements of TCC. The levels of TCC were undetectable at the start of the experiments but increased steadily in all tubes throughout the experiment. At several time points significantly higher levels of TCC could be detected in heparin coated tubes compared to PMEA coated tubes (at 75 min: $7.5 \pm 0.8 \mathrm{AU} / \mathrm{mL}$ for heparin coated tubes vs $4.8 \pm 0.2 \mathrm{AU} / \mathrm{mL}$ for PMEA coated tubes (mean \pm sem), $\mathrm{p}=0.001$; at $150 \mathrm{~min}: 11.5 \pm 1.4 \mathrm{AU} / \mathrm{mL}$ for heparin coated tubes vs $7.9 \pm 0.4 \mathrm{AU} / \mathrm{mL}$ for PMEA coated tubes (mean \pm sem), $\mathrm{p}=0.024$; at $225 \mathrm{~min}: 14.7 \pm 1.3 \mathrm{AU} / \mathrm{mL}$ for heparin coated tubes vs 11.1 $\pm 0.7 \mathrm{AU} / \mathrm{mL}$ for PMEA coated tubes (mean $\pm \mathrm{sem}), \mathrm{p}=0.027$ ) and uncoated controls (at $75 \mathrm{~min}: 7.5 \pm 0.8 \mathrm{AU} / \mathrm{mL}$ for heparin coated tubes vs $5.9 \pm 0.7$ $\mathrm{AU} / \mathrm{mL}$ for uncoated controls (mean \pm sem), $\mathrm{p}=0.036$ ). This suggests that TCC generation proceeds faster in heparin coated tubes compared to PMEA coated tubes and uncoated controls. Several in vitro studies using the Chandler loop model reported prevention of TCC generation by heparin coated PVC compared to uncoated PVC 20,22,29,33. However, none of these studies had blood circulation times of more than two hours. Also, these studies used heparin coatings which were structurally different from the heparin coating evaluated in our study. Weber et al. compared covalently heparin coated tubes from four different manufacturers and also found marked differences in hemocompatibility ${ }^{11}$.

\section{Analysis of the inner surface}

\section{$\underline{\text { Scanning electron microscopy }}$}

A set of scanning electron micrographs was recorded (3 different materials, 4 donors, 5 different times of circulation in the Chandler loop system, and three samples of every tube were examined). In general, we observed that adhesion of blood components developed slowly and gradually as the experiments proceeded. However, the SEM data revealed a striking difference between uncoated PVC and heparin-coated PVC on one hand, and the PMEAcoated PVC on the other hand. Four micrographs, taken after $5 \mathrm{~h}$ of blood circulation over the surfaces, are shown in Figure 6 to illustrate this difference. The uncoated PVC and heparin-coated PVC surfaces showed a remarkable resemblance. These surfaces were, to an extent of approximately $80 \%$, devoid of any visible adherent blood components. There were, however, island-like regions, usually small (e.g. $10 \times 10$ m) but sometimes larger (e.g. $100 \times 200$ m). Enlarged images of these islands (Fig. 6A and 6B) revealed a flat patch-like structure, presumably composed of fibrin threads. Some blood platelets were entrapped in the patch, in the case of the uncoated surface (Fig. 6A). For the heparin-coated surface, platelets as well as larger cells (presumably leukocytes) 
were entrapped in or adhered to the patch structure (Fig. 6B). Evaluation of the inner surface of the PMEA-coated tubing showed radically different pictures, as can be seen in Figure 6C and 6D. Fibrin formation is evident on the uncoated and heparin-coated surfaces, but not for the PMEA-coating. Scattered over the PMEA coated surface, we encountered isolated cells, or ensembles of a small number of cells (typically 2 or 3 cells). Presumably, the adhered cells are leukocytes; the diameter of these cells is $10-15 \mathrm{~m}$. Figure 6D shows a detailed SEM micrograph of a region that was relatively densely populated with adherent cells. It is seen that most of the cells extend pseudopodia, through which they are connected to one or more neighbors. Leukocyte activation induced by surface contact, leading to pseudopodia (Fig. 6D), can also explain why the MPO concentration was highest for the PMEA-coated PVC tube, as compared to the other two tubes (vide supra, Figure 4).
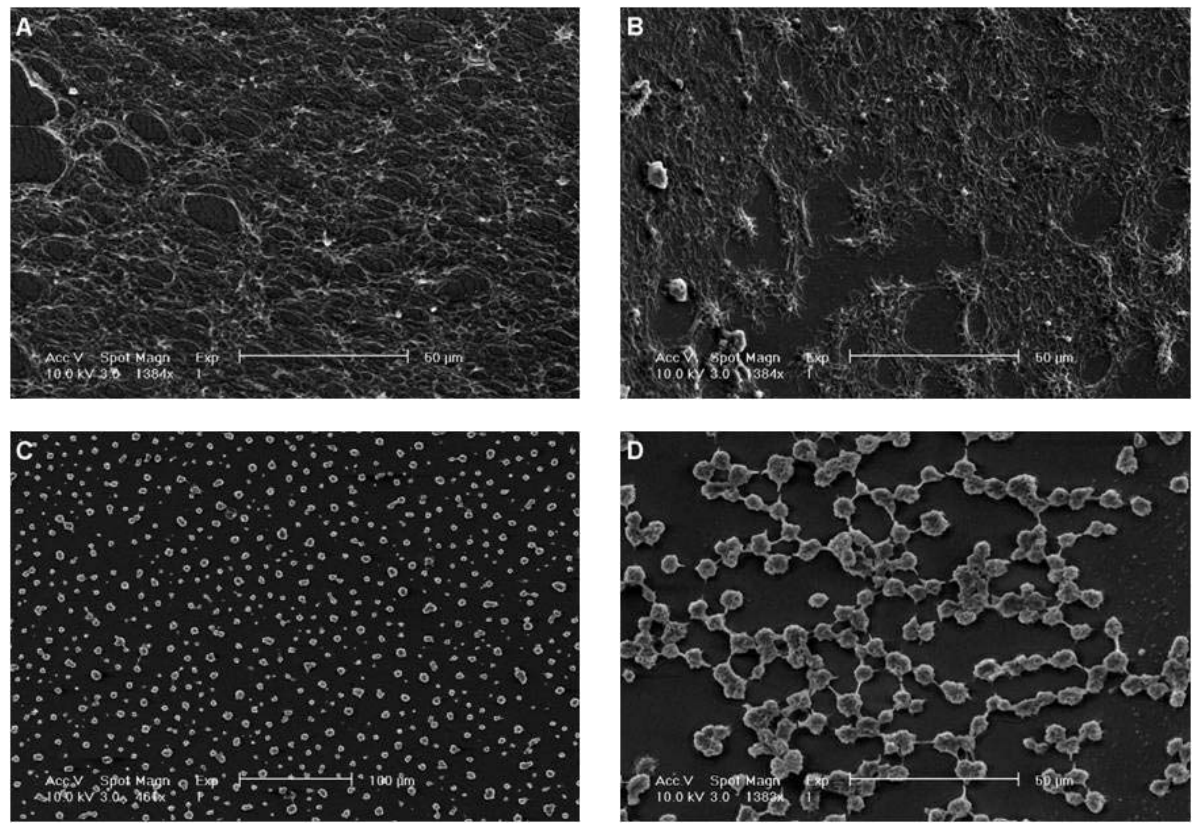

Figure 6. A: Scanning electron micrograph of a typical island structure on the inner surface of the uncoated (control) tube, after $5 \mathrm{~h}$ contact with full human blood in the Chandler loop model. A patch-like structure is seen, which is -presumably- composed of fibrin threads, which adhered to the surface. B: Scanning electron micrograph of a typical island structure on the inner surface of the heparin-coated tube (same conditions). The patch-like structure in A and B are similar; note that platelets as well as cells (presumably leukocytes) are seen in B. C: Scanning electron micrograph of the PMEA coated surface, after $5 \mathrm{~h}$ of experimentation in the Chandler loop. Adhered cells are observed, either individually, or in small aggregates of 2 or 3 cells. Cells and aggregates are, to a good approximation, evenly spread over the surface, although regions of relatively high cell density could be discerned. D: Close-up of a region of relatively high cell density. Note that most of the adherent cells are engaged into cell-cell contacts through pseudopodia. 


\section{Measurement of thrombin generation times}

In view of the differences between these coatings encountered after prolonged blood contact in the Chandler loop, we decided to subject the surfaces also to the well-known thrombin generation assay ${ }^{16,17,18,19}$. Contact activation of the blood coagulation system is accompanied by a sudden increase of thrombin levels, after a lag-time that varies between approximately 5 min for highly thrombogenic materials, to approximately $60 \mathrm{~min}$ for materials with extremely low thrombogenicity. Figure 7 shows the thrombin generation curves measured in triplicate with the uncoated, heparin coated and PMEA coated PVC tubes. Thrombin generation occurred between 30 and $40 \mathrm{~min}$ in the uncoated PVC tubes. Coating of the PVC with immobilized heparin prolonged the thrombin generation time until $60 \mathrm{~min}$. This clearly indicates the better antithrombogenic properties of the heparin coated PVC tubes compared to uncoated PVC tubes. A remarkable finding, however, was that coating of PVC tubes with PMEA resulted in thrombin generation times comparable to those of the uncoated PVC tubes. Note that, in the case of the heparin-coated PVC, the thrombin concentration remains $<2 \mathrm{nM}$, even after $60 \mathrm{~min}$. The analysis was stopped after this time point, since the assay is static. The red blood cells may show aberrant behavior after $1 \mathrm{~h}$ of static conditions (Rouleaux formation), which compromises the reliability of the method. Comparing the thrombin generation curves, we conclude that thrombogenicity of the three different surfaces is as follows: uncoated $\mathrm{PVC} \approx \mathrm{PMEA}$-coated $\mathrm{PVC}>$ heparin-coated PVC.
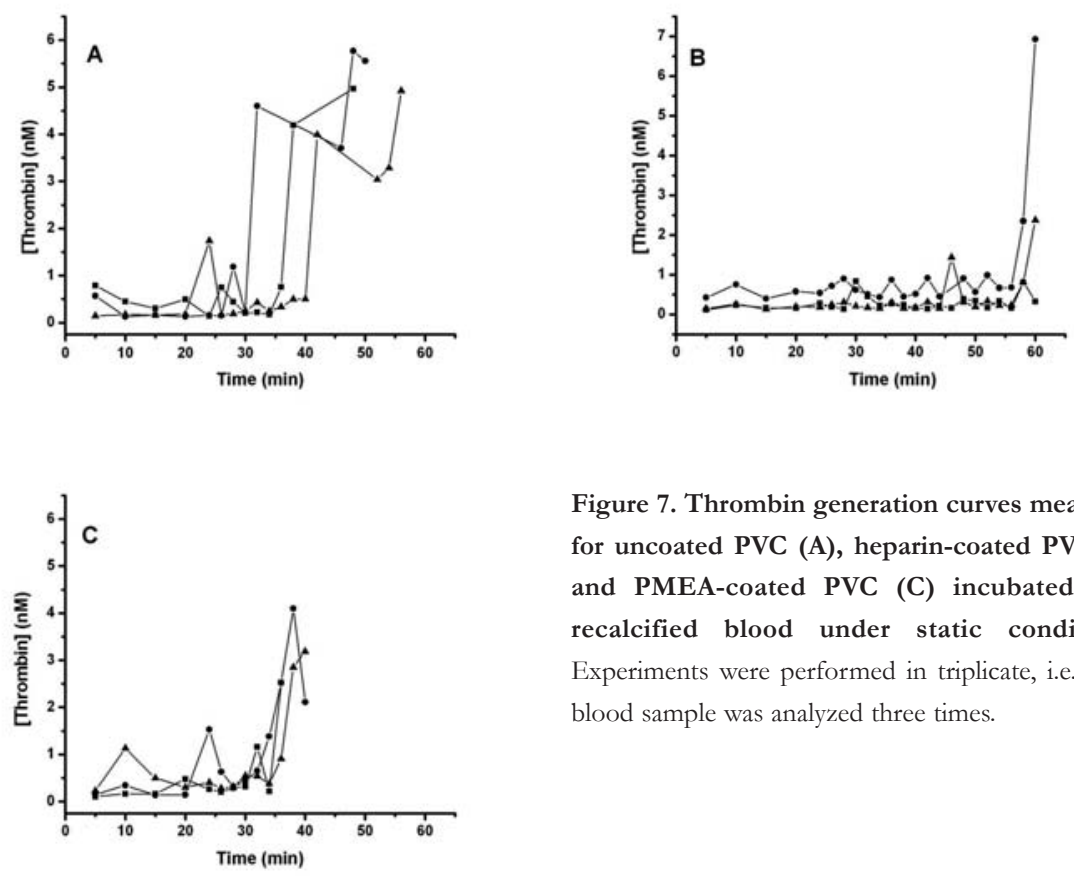

Figure 7. Thrombin generation curves measured for uncoated PVC (A), heparin-coated PVC (B) and PMEA-coated PVC (C) incubated with recalcified blood under static conditions. Experiments were performed in triplicate, i.e. every blood sample was analyzed three times. 


\section{The Chandler loop model}

After collecting all data, we wondered whether any correlation could be defined between the intensity of blood-biomaterial contact in the Chandler loop system, and that in real-life extracorporeal circulation in CPB. Assuming that the level of blood-biomaterial contact (Q) is proportional to the surface area of the artificial material $(\mathrm{S})$ and the time of blood-biomaterial contact $(\mathrm{t})$, and inversely proportional to the blood volume $(\mathrm{V})$, it can be calculated that the Chandler loop system (with $\mathrm{S}=40 \mathrm{~cm}^{2}, \mathrm{~V}=7 \mathrm{~mL}, \mathrm{t}=5 \mathrm{~h}$ ), that $\mathrm{Q}=28.6 \mathrm{~h} / \mathrm{cm}$. For a typical CPB system (with $\mathrm{S}=25,000 \mathrm{~cm}^{2}, \mathrm{~V}=8,000 \mathrm{~mL}(6 \mathrm{~L}$ of blood $+2 \mathrm{~L}$ of priming fluid $)$, it then follows that $\mathrm{t}=(28.6 \times 8,000) / 25,000 \approx 9 \mathrm{~h}$. In other words, this simple model indicates that $5 \mathrm{~h}$ of experimentation in the Chandler loop corresponds with a level of blood-biomaterial contact that corresponds to at least $9 \mathrm{~h}$ of operation in a typical CPB system. Most probably, the estimated $9 \mathrm{~h}$ is an underestimation, since the blood cells in real-life CPB are oxygenated and the blood resides mostly within the patient's vasculature. This probably implies that some recuperation of bloods cells occurs during real-life CPB, while this is evidently not the case in our model system. Noteworthy, the extent of hemolysis encountered in our experiments was very low, i.e., $<0.3 \%$ after $5 \mathrm{~h}$ of circulation, irrespective of the donor or (coated) surface.

\section{Conclusion}

Systematic evaluation of the blood biomaterial contact for the three different tubings, using relatively long periods of blood-biomaterial contact, was performed. The three different surfaces were: uncoated PVC, heparin-coated PVC, and PMEA-coated PVC; the latter two are already in clinical use, as tubings in extracoporeal circulation equipment. Clear differences with respect to platelet counts, leukocyte activation (MPO release), and deposition of blood components at the inner surfaces were found. Most of these differences became apparent only after the first $2-3 \mathrm{~h}$ of experimentation. This underlines the importance of extended evaluation of blood-contacting biomaterials that are to be used in long-term applications, such as extended CPB. It is noteworthy that the PMEA coated tubes showed a relatively low level of hemocompatibility. Compared with uncoated PVC and heparin-coated PVC, (i) a substantial drop of the platelet counts, (ii) activation of leukocytes and marked adherence of leukocytes at the inner surface and (iii) a thrombogenicity comparable to uncoated PVC, were observed. The present results lead to two important conclusions:

The Chandler loop system is a most useful method for the evaluation of blood-biomaterial interactions, which is most probably relevant for the development of equipment for extracorporeal circulation. Extended 
experimentation times (e.g. $5 \mathrm{~h}$ ) are mandatory, since differences for various materials may masquerade during the first few hours. PMEA biomaterial is probably not optimal for use in extracorporeal circulation equipment; further improvements are necessary.

\section{Acknowledgements}

The help of $\mathrm{mr} \mathrm{H}$. Jussen and $\mathrm{mr}$ F. Spee (Instument Development Engineering and Evaluation, University of Maastricht) is gratefully acknowledged. This study was financed within the framework of the Bioterials program, a 4-years private-public joint effort of the Dutch Ministry of Economic Affairs, the Province of Dutch Limburg, DSM Research BV (Geleen, the Netherlands), the Academic Hospital Maastricht, and the University of Maastricht. Bioterials runs in the period April 2005 - April 2009. 


\section{References}

1 Mangoush, O. et al. Heparin-bonded circuits versus nonheparin-bonded circuits: an evaluation of their effect on clinical outcomes. Eur J Cardiothorac Surg 31, 1058-1069, (2007).

2 Rubens, F. D. Cardiopulmonary bypass technology transfer: musings of a cardiac surgeon. J Biomater Sci Polym Ed 13, 485-499 (2002).

3 Skinner, S. C., Hirschl, R. B. \& Bartlett, R. H. Extracorporeal life support. Semin Pediatr Surg 15, 242-250, (2006).

4 Wendel, H. P. \& Ziemer, G. Coating-techniques to improve the hemocompatibility of artificial devices used for extracorporeal circulation. Eur J Cardiothorac Surg 16, 342-350, (1999).

5 Baykut, D. et al. New surface biopolymers for oxygenators: an in vitro hemocompatibility test of poly(2-methoxyethylacrylate). Eur J Med Res 6, 297-305 (2001).

6 Mueller, X. M., Jegger, D., Augstburger, M., Horisberger, J. \& von Segesser, L. K. Poly2-methoxyethylacrylate (PMEA) coated oxygenator: an ex vivo study. Int J Artif Organs 25, 223-229 (2002).

7 Ninomiya, M., Miyaji, K. \& Takamoto, S. Influence of PMEA-coated bypass circuits on perioperative inflammatory response. Ann Thorac Surg 75, 913-917; discussion 917-918 (2003).

8 Mason, D. P. et al. Extended use of extracorporeal membrane oxygenation after lung transplantation. J Thorac Cardiovasc Surg 132, 954-960, (2006).

9 O'Neill, J. M., Schutze, G. E., Heulitt, M. J., Simpson, P. M. \& Taylor, B. J. Nosocomial infections during extracorporeal membrane oxygenation. Intensive Care Med 27, 1247-1253 (2001).

10 Sung, K. et al. Improved survival after cardiac arrest using emergent autopriming percutaneous cardiopulmonary support. Ann Thorac Surg 82, 651-656, (2006).

11 Weber, N., Wendel, H. P. \& Ziemer, G. Quality assessment of heparin coatings by their binding capacities of coagulation and complement enzymes. J Biomater Appl 15, 8-22 (2000).

12 Rijkers, D. T., Wielders, S. J., Tesser, G. I. \& Hemker, H. C. Design and synthesis of thrombin substrates with modified kinetic parameters. Thromb Res 79, 491-499 (1995).

13 Chandler, A. B. In vitro thrombotic coagulation of the blood; a method for producing a thrombus. Lab Invest 7, 110-114 (1958).

14 Kopp, R. et al. Effect of hirudin versus heparin on hemocompatibility of blood contacting biomaterials: an in vitro study. Int J Artif Organs 28, 1272-1277 (2005).

15 Cripps, C. M. Rapid method for the estimation of plasma haemoglobin levels. J Clin Pathol 21, 110-112 (1968).

16 Aldenhoff, Y. B., Blezer, R., Lindhout, T. \& Koole, L. H. Photo-immobilization of dipyridamole (Persantin) at the surface of polyurethane biomaterials: reduction of in-vitro thrombogenicity. Biomaterials 18, 167-172, (1997).

17 Aldenhoff, Y. B. et al. Coils and tubes releasing heparin. Studies on a new vascular graft prototype. Biomaterials 25, 3125-3133, (2004).

18 Barrowcliffe, T. W. et al. New approaches for measuring coagulation. Haemophilia 12 Suppl 3, 76-81, (2006).

19 van Oeveren, W., Haan, J., Lagerman, P. \& Schoen, P. Comparison of coagulation activity tests in vitro for selected biomaterials. Artif Organs 26, 506-511, (2002).

20 Weber, N., Wendel, H. P. \& Ziemer, G. Hemocompatibility of heparin-coated surfaces and the role of selective plasma protein adsorption. Biomaterials 23, 429-439 (2002).

21 Sreeram, G. M., Sharma, A. D. \& Slaughter, T. F. Platelet glycoprotein IIb/IIIa antagonists: perioperative implications. J Cardiothorac Vasc Anesth 15, 237-240, (2001).

22 Lappegard, K. T. et al. Effect of complement inhibition and heparin coating on artificial surface-induced leukocyte and platelet activation. Ann Thorac Surg 77, 932-941, (2004). 
23 Rinder, C. S. et al. Cardiopulmonary bypass induces leukocyte-platelet adhesion. Blood 79, 1201-1205 (1992).

24 Wu, Y. P., de Groot, P. G. \& Sixma, J. J. Shear-stress-induced detachment of blood platelets from various surfaces. Arterioscler Thromb Vasc Biol 17, 3202-3207 (1997).

25 Kawahito, K., Mohara, J., Misawa, Y. \& Fuse, K. Platelet damage caused by the centrifugal pump: in vitro evaluation by measuring the release of alpha-granule packing proteins. Artif Organs 21, 1105-1109 (1997).

26 Kutay, V. et al. Biocompatibility of heparin-coated cardiopulmonary bypass circuits in coronary patients with left ventricular dysfunction is superior to PMEA-coated circuits. J Card Surg 21, 572-577, (2006).

27 Malinauskas, R. A. Plasma hemoglobin measurement techniques for the in vitro evaluation of blood damage caused by medical devices. Artif Organs 21, 1255-1267 (1997).

28 Ohkawa, R. et al. Platelet release of beta-thromboglobulin and platelet factor 4 and serotonin in plasma samples. Clin Biochem 38, 1023-1026, (2005).

29 Lappegard, K. T., Fung, M., Bergseth, G., Riesenfeld, J. \& Mollnes, T. E. Artificial surfaceinduced cytokine synthesis: effect of heparin coating and complement inhibition. Ann Thorac Surg 78, 38-44; discussion 44-35, (2004).

30 Asberg, A. E. \& Videm, V. Activation of neutrophil granulocytes in an in vitro model of a cardiopulmonary bypass. Artif Organs 29, 927-936, (2005).

31 Asberg, A. E. \& Videm, V. Neutrophil dysfunction after biomaterial contact in an in vitro model of cardiopulmonary bypass. Eur J Cardiothorac Surg 30, 744-748, (2006).

32 Gong, J. et al. Tubing loops as a model for cardiopulmonary bypass circuits: both the biomaterial and the blood-gas phase interfaces induce complement activation in an in vitro model. J Clin Immunol 16, 222-229 (1996).

33 Andersson, J. et al. Optimal heparin surface concentration and antithrombin binding capacity as evaluated with human non-anticoagulated blood in vitro. J Biomed Mater Res A 67, 458-466, (2003). 


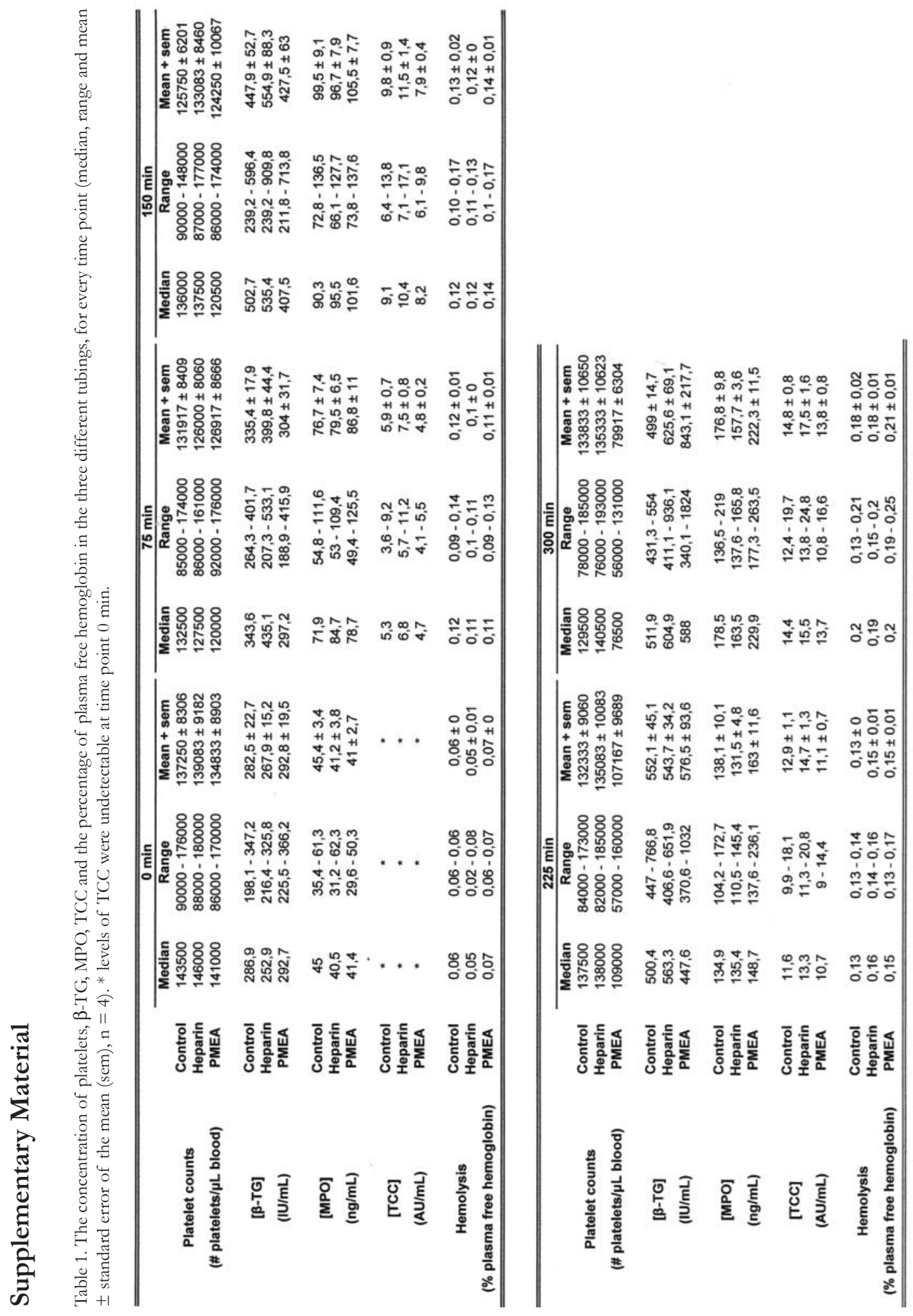


The relationship between the antimicrobial effect of catheter coatings containing silver nanoparticles and the coagulation of contacting blood

Stevens $\mathrm{KN}^{1}$, Crespo-Biel $\mathrm{O}^{2}$, van den Bosch $\mathrm{EE}^{2}$, Dias $\mathrm{AA}^{2}$, Knetsch $\mathrm{ML}^{3}$, Aldenhoff $\mathrm{YB}^{3}$, van der Veen $\mathrm{FH}^{1}$, Maessen $\mathrm{JG}^{1}$, Stobberingh EE ${ }^{4}$, Koole $\mathrm{LH}^{3}$.

1. Department of Cardiothoracic Surgery, Maastricht University Medical Centre, Maastricht, The Netherlands.

2. DSM Research, Geleen, The Netherlands.

3. Centre for Biomaterials Research, University of Maastricht, Maastricht, The Netherlands.

4. Department of Medical Microbiology, Maastricht University Medical Centre, Maastricht, The Netherlands.

Biomaterials. 2009;30(22):3682-90. 


\section{Abstract}

It is well known that surface coatings for medical devices can be made antimicrobial through introduction of silver nanoparticles. By virtue of their extremely large surface-to-volume ratio, the silver particles serve as a depot for sustained release of silver ions, despite the fact that silver is not readily oxidized. Antimicrobial coatings are especially important in connection with indwelling catheters with a high risk of bacterial line infections, such as central venous catheters (CVCs). This study specifically addressed the question what the impact of silver nanoparticles (exposed at the coating's surface) and/or the release of silver ions would be on coagulation of contacting blood. Studies, performed in vitro with fresh platelet-rich blood plasma (PRP) from 5 different healthy volunteer donors, clearly pointed out that: (i), the presence of silver nanoparticles correlates with accelerated thrombin formation upon contact of the coating with PRP; (ii), platelet activation is stronger as a result from the contact with silver nanoparticle-containing coatings as compared to other coatings which are devoid of silver. A series of titration experiments, in which the potential effect of silver ions is mimicked, revealed that the observed activation of blood platelets can be best explained through a collision mechanism. The results suggest that platelets that collide with silver, exposed at the surface, become activated without adhering to the surface. These new results point, rather unexpectedly, at a double effect of the silver nanoparticles in the coating: a strong antimicrobial effect occurs simultaneously with acceleration of the coagulation of contacting blood. This new information is, evidently, most relevant for the development of improved surface coatings for indwelling catheters (such as CVCs) which should combine antimicrobial features and close-to-zero thrombogenicity. 


\section{Introduction}

The recent definition of the term nanomaterial ("any form of a material that is composed of discrete functional particles, many of which has one or more dimensions in the order of $100 \mathrm{~nm}$ or less") ${ }^{1}$ clarifies that only very few bio-nanomaterials are currently in use. Perhaps the most prominent example is provided by a new class of polymeric medical device coatings, i.e. those that contain silver nanoparticles. These coatings feature clear antimicrobial properties in vivo, as they slowly release $\mathrm{Ag}^{+2,3}$. Although silver (being a halfnoble metal) is not easily oxidized, the enormous surface-to-volume ratio of the nanoparticles accounts for a sustained local supply of $\mathrm{Ag}^{+}$at the coating-tissue interface ${ }^{4}$. This effect is held responsible for prolonged prevention of bacterial adhesion and biofilm formation. The use of silver-containing nanomaterials is regarded as one of the most promising strategies to combat bacterial infections related to indwelling medical devices, e.g., catheters. Such infections are an important public health issue, ranking as the fifth-leading cause of hospital patients deaths in the US ${ }^{5}$.

Recently, we became interested in silver-containing nanomaterials, especially with a view on their potential utility as surface-coatings for central venous catheters (CVCs). It is well known that the use of CVCs is associated with two major problems: (i) bacterial line-infection, and (ii), thrombus formation ${ }^{6,7}$. According to Wenzel and Edmond ${ }^{8}$ CVCs are used throughout $54 \%$ of the days that patients are in intensive care units. It has been estimated that CVC-associated bloodstream infections (primarily of coagulase-negative staphylococci, Staphylococcus aureus, enterococcus species and candida species) occur with a frequency of 5 per 1000 catheter-days ${ }^{9}$. We set out to investigate whether the use of silver nanoparticles as a part of the surface coating would interfere with the thrombogenicity of the catheter's surface. A priori, based on our previous research work on thrombogenicity of hydrophilic surface coatings, we expected that the presence of silver particles at the blood-biomaterial interface might have a thrombogenic effect.

Here, we report an investigation on silicone rubber catheter samples that were coated with an adherent hydrophilic polymer coating. We prepared two series of samples, in which we incorporated silver nanoparticles in the coating (either $8 \%$ or $15 \%$ by dry mass). Comparisons were made with: (i) catheter tubes which were coated with the hydrophilic polymer coating, but without silver; (ii) catheters without a surface coating (silicone and PVC tubing); (iii) several commercial catheters, having either a hemocompatible or an antimicrobial surface coating. We studied all materials in contact with fresh human platelet-rich plasma (PRP) from 5 different volunteer donors. The results are discussed in the context of further developments that should lead to novel catheter coatings that effectively combine prevention of bacterial line infections and close-to-zero thrombogenicity. 


\section{Materials \& methods}

\section{Materials}

Throughout this study, 8 different catheter samples were used. Some were commercially available catheters, some were silicone tubes that were coated by ourselves, and some were uncoated tubes, serving as controls. Table 1 provides a comprehensive overview.

$\mathrm{NaCl}, \mathrm{CaCl} 2 \cdot(\mathrm{H} 2 \mathrm{O}) 2$, glutaraldehyde 25\%, dimethylsulfoxide (DMSO) and Triton X-100, were purchased from Acros Organics (Geel, Belgium). Trisodium citrate and K2HPO4 were products from Sigma-Aldrich Chemie B.V. (Zwijndrecht, The Netherlands). KH2PO4 and AgNO3 were from Janssen Chimica (Beerse, Belgium). Ethanol 100\%, nitric acid (p.a. 65\%), silver stock solution (for silver quantification), H2O2 (p.a. 30\%), hydrochloric acid (p.a. $37 \%$, palladium matrixmodifier and $\mathrm{Mg}(\mathrm{NO} 3) 2 \cdot(\mathrm{H} 2 \mathrm{O}) 6$ were supplied by Merck KGaA (Darmstadt, Germany). Lepirudin (Refludan) was obtained from Pharmion (Windsor Berkshire, UK). The fluorogenic substrate for thrombin, ZGly-Gly-Arg-AMC, was a product of Bachem Holding AG (Bubendorf, Switzerland; ref. I-1140). The platelet-stabilizing anticoagulant mixture, citratetheophylline-adenosine-dipyridamole (CTAD), was purchased from Becton Dickinson (Alphen aan den Rijn, The Netherlands). Brain Heart Infusion Broth and Mueller-Hinton agar were from Oxoid B.V. (Badhoevedorp, The Netherlands). The lactate dehydrogenase (LDH) assay was performed using the CytoTox 96® Non-Radioactive Cytotoxicity Assay from Promega Benelux B.V. (Leiden, The Netherlands). Platelet activation was quantified with the Asserachrom ${ }^{\circledR} \beta$-TG enzyme-linked immunosorbent assay (ELISA), obtained from Roche Diagnostics Nederland B.V. (Almere, The Netherlands). 
Table 1. Overview of the catheter materials used throughout this study. (a) Purchased from Vygon Nederland B.V. (Valkenswaard, The Netherlands; ref. 8157.207). (b) Purchased from Bard Nordic (Helsingborg, Sweden; ref. 5664190). (c) Purchased from Medtronic Nederland (Heerlen, The Netherlands; ref. M311902A). (d) Purchased from Medos Medizintechnik AG (Stolberg, Germany; ref. 039682). (e) Purchased from Raumedic AG (Münchberg, Germany; ref. 819362-001) (f) Prepared as a part of this study.

\begin{tabular}{|c|c|c|c|c|}
\hline Catheter & $\begin{array}{l}\text { Commercial } \\
\text { source }\end{array}$ & Coating & $\begin{array}{l}\text { Inner Diameter } \\
(\mathrm{mm})\end{array}$ & $\begin{array}{l}\text { Outer Diameter } \\
(\mathrm{mm})\end{array}$ \\
\hline Vygon ${ }^{\circledR}(\mathrm{a})$ & Yes & $\begin{array}{l}\text { antimicrobial AgION } \\
\text { technology }\left(\mathrm{Ag}^{+}\right. \\
\text {integrated into the } \\
\text { polyurethane substrate) }\end{array}$ & $\begin{array}{l}2 \text { lumens; distal } 16 \mathrm{G} \\
\text { and proximal } 18 \mathrm{G}\end{array}$ & 2.50 \\
\hline HemoGlide $^{\mathrm{TM}}$ (b) & Yes & No & $\begin{array}{l}2 \text { lumens; } \\
2.30 \mathrm{~mm} \text { each }\end{array}$ & 4.83 \\
\hline PVC-Carmeda ${ }^{\circledR}$ (c) & Yes & $\begin{array}{l}\text { antithrombotic } \\
\text { Carmeda }{ }^{\circledR} \text { (heparin) }\end{array}$ & 4.76 & 7.94 \\
\hline PVC (d) & Yes & No & 4.76 & 7.94 \\
\hline Sil (e) & Yes & No & 3.43 & 5.48 \\
\hline Sil-PVP & No & hydrophilic coating (f) & 3.43 & 5.48 \\
\hline Sil-PVP-Ag(8) & No & $\begin{array}{l}\text { hydrophilic coating } \\
\text { containing silver } \\
\text { nanoparticles (f) }\end{array}$ & 3.43 & 5.48 \\
\hline Sil-PVP-Ag(15) & No & $\begin{array}{l}\text { hydrophilic coating } \\
\text { containing silver } \\
\text { nanoparticles (f) }\end{array}$ & 3.43 & 5.48 \\
\hline
\end{tabular}

\section{Equipment}

Fluorescence tracings were recorded on a SpectraMax M2 spectrofluorometer (Molecular Devices, Sunnyvale, CA, USA). Absorbances were measured on an ELx808 Absorbance Microplate Reader (BioTek Instruments, Inc., VT, USA). Sputter coating was performed with a Sputter coater 108 auto/SE (Cressington Scientific Instruments Ltd., Watford, UK) and for scanning electron microscopy (SEM) analysis, a Philips XL30 Scanning Electron Microscope (Philips, Eindhoven, The Netherlands) was used. Centrifugation was performed in a MSE Mistral 3000 I centrifuge (Beun - de Ronde, Abcoude, The Netherlands). Silver quantification was performed by means of a graphite furnace (Varian) atomic absorption spectrophotometer (Spectra 400 Zeeman, Varian, Palo Alto, CA, USA). 


\section{Test samples}

Silicone tubing was dual coated with a lubricious, silver nanoparticle containing coating. Coating application and characterization were described in detail in patent WO2007/065721 ${ }^{10}$. Briefly, the outer surface and lumen of tubes were successively coated with the primer and topcoat formulations by a dip coating procedure involving UV radiation curing. This results in homogeneous, 6-12 $\mu \mathrm{m}$ thick, hydrophilic lubricious coatings. A uniform distribution of silver nanoparticles throughout the coatings was obtained ${ }^{10}$. Silver nanoparticles were prepared by sodium borohydride reduction of silver nitrate as described by Jin, et $\mathrm{al}^{11}$. A monodisperse suspension of silver particles with an average diameter of $20 \mathrm{~nm}$ was obtained.

\section{Preparation of catbeter samples}

Catheter samples were cut to the correct dimensions (vide infra) in a laminar flow cabinet. Catheters Sil and Sil-PVP were used as references in all of our experiments; PVC and PVC-Carmeda ${ }^{\circledR}$ were used as additional clinical references in our experiments to measure platelet activation and platelet adhesion, as well as throughout our SEM studies. The Vygon ${ }^{\circledR}$ catheter was used as an additional reference in our bacterial adhesion experiments, and HemoGlide ${ }^{\mathrm{TM}}$ was used as an extra reference in the thrombin generation assay. In most of our experiments we used the catheter samples directly as incubation wells by closing one end with a surgical tube clamp. The dimensions of the HemoGlide ${ }^{\mathbf{T M}}$ and Vygon ${ }^{\circledR}$ catheters were too small to apply this method.

\section{Antimicrobial properties}

Prior to the antimicrobial experiment, catheter samples (length: $3 \mathrm{~cm}$ ) were either left unwashed or were incubated either 1 or 7 days in $10 \mathrm{~mL}$ Phosphate Buffered Saline (PBS) (10 mM K2HPO4, 10 mM KH2PO4, 150 mM $\mathrm{NaCl} ; \mathrm{pH} 7$ ), at $37^{\circ} \mathrm{C}$ in a shaking incubator. PBS was replaced after 2,4 and 6 h on day 1 and daily afterwards. An overnight (ON)-culture of Staphylococcus aureus ATCC 29213 was prepared by inoculation of one bacterial colony into 10 mL growth medium (37 g/L Brain Heart Infusion Broth) and ON incubation at $37^{\circ} \mathrm{C}$. From this culture, a bacterial suspension of $107 \mathrm{CFU} / \mathrm{mL}$ was prepared in $0.9 \% \mathrm{NaCl}$.

Catheter samples were incubated $1 \mathrm{~h}$ in $5 \mathrm{~mL}$ bacterial suspension, in a shaking incubator at $37^{\circ} \mathrm{C}$. The samples were subsequently transferred to a fresh volume of $5 \mathrm{~mL} 0.9 \% \mathrm{NaCl}$ and vortexed $5 \mathrm{~s}$ to remove loosely adhered bacteria. Washed catheter samples were transferred again to a fresh volume of 5 $\mathrm{mL} 0.9 \% \mathrm{NaCl}$ and incubated $4 \mathrm{~h}$ at $37^{\circ} \mathrm{C}$ under continuous shaking. Finally, the 
catheter samples were rinsed in PBS and rolled over a Mueller-Hinton blood agar plate (38 g/L Mueller-Hinton agar, 5\% defibrinated sheep blood) by the method of Maki, et al ${ }^{12}$. Plates were incubated $\mathrm{ON}$ at $37^{\circ} \mathrm{C}$ and evaluated the next day. All catheter groups were tested in triplo.

\section{Interaction with $P R P$}

\section{Blood collection and preparation of PRP}

These experiments were executed with freshly prepared human PRP from 5 healthy male volunteers. The PRP served as a simplified environment, combining the two main players of the hemostatic mechanism, i.e., platelets and clotting factors. Blood was withdrawn from healthy human volunteers by venipuncture. The donors were all non-smokers and did not take any hemostasis-influencing medicines at least 10 days before the experiment. Depending on the experiment to be performed, the blood was immediately anticoagulated with either citrate (end-concentration: $0.013 \mathrm{M}$ citrate), or lepirudin, a recombinant form of hirudin (end-concentration: $20 \mu \mathrm{g} / \mathrm{mL}$ lepirudin). Lepirudin is a direct thrombin inhibitor and executes its anticoagulant effect without interfering with platelet function ${ }^{13}$. Therefore it is the anticoagulant of choice for platelet activation studies. To isolate PRP, the anticoagulated whole blood was centrifuged $15 \mathrm{~min}$ at room temperature and $200 \mathrm{x}$ g. The PRP fraction was then pipetted into a fresh tube and kept at $37^{\circ} \mathrm{C}$ until further use.

\section{Thrombin generation assay}

Each thrombin generation experiment was performed with PRP from a single donor. This allowed all catheter groups to be tested with identical PRP. The experiment was performed five times with PRP from five different donors. In each experiment, every catheter group was tested in six-fold. Catheter samples (length: $0.4 \mathrm{~cm}$ ) were placed horizontally in the wells of a 96 well-plate as shown in Figure 1.

Just before the start of the experiment, a fluorogenic substrate for thrombin, Z-Gly-Gly-Arg-AMC, was added to citrated PRP to a final concentration of $400 \mu \mathrm{M}$. Next, the citrated PRP was "recalcified" by adding $\mathrm{CaCl} 2$ stock solution $(0.5 \mathrm{M} \mathrm{CaCl} 2)$ to a final concentration of $20 \mathrm{mM} \mathrm{CaCl} 2$. Upon recalcification, concentrations of $\mathrm{Ca} 2+$ were restored to physiological levels, and the blood clot mechanism is no longer inhibited. Then, PRP + fluorogenic substrate was instantly distributed over the wells in volumes of $200 \mu \mathrm{L}$. Wells without a catheter sample served as controls. Fluorescence tracings were recorded at $37^{\circ} \mathrm{C}$, every 30 seconds during $1 \mathrm{~h}\left(\lambda_{\mathrm{ex}}=368 \mathrm{~nm}\right.$ and $\lambda_{\mathrm{em}}=460 \mathrm{~nm}$ and $2 \mathrm{~s}$ shaking prior to each measurement) ${ }^{14}$. The fluorescence 
intensity was converted into nanomolar concentrations thrombin as described earlier by Hemker, et $\mathrm{al}^{15}$. This resulted in a thrombin generation curve for each well, as shown in Figure 2.

a

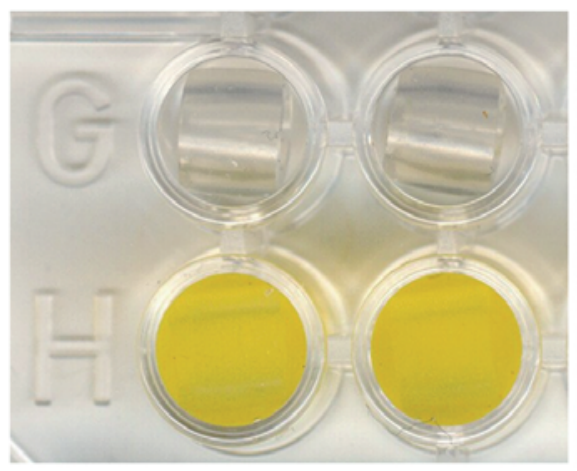

b

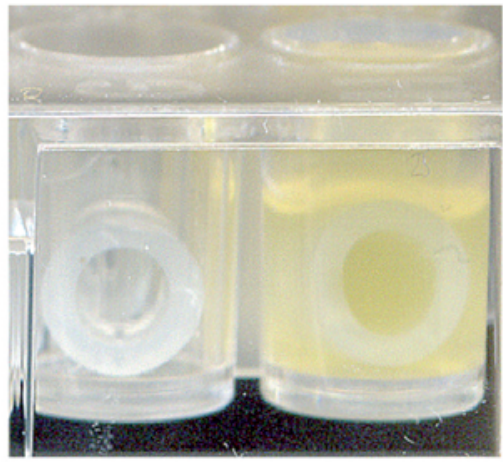

Figure 1. (a) Horizontal placement of the catheter samples in the wells of a 96 well-plate (above view). The wells in row $\mathrm{H}$ are filled with PRP. (b) Side view of two wells filled with horizontally placed catheter samples; the well on the right is filled with PRP.
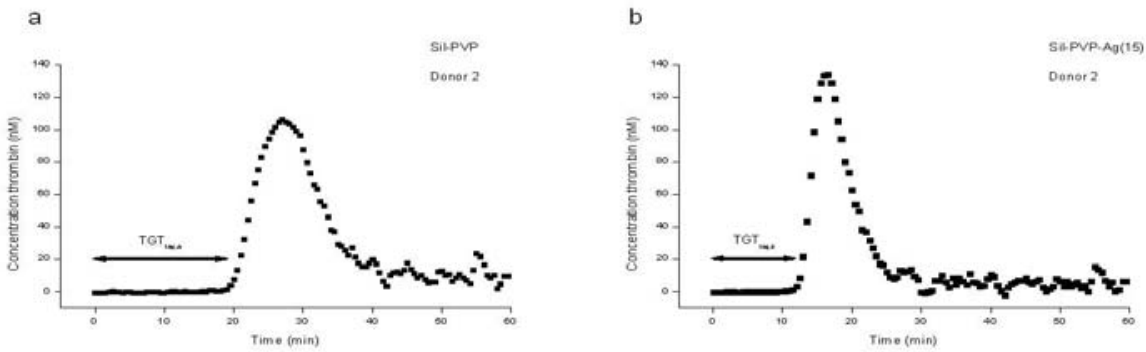

Figure 2. Thrombin generation curves measured with a Sil-PVP sample present in the well (a) and with a SilPVP-Ag(15) sample present in the well (b). Both experiments were performed simultaneously with PRP from a single donor (Donor 2). Notice how the time span between the onset of the experiment and the sudden rise in thrombin levels (TGTlag,a and TGTlag,b) differs for the different catheter samples.

A thrombin generation curve plots the thrombin concentration vs time. During the first minutes thrombin levels remain close to zero. Then, a sudden increase in thrombin levels is seen. The thrombin concentration rises steeply, passes a maximum and slowly decreases back to (approximately) zero. This is the result of the simultaneous occurrence of thrombin formation and thrombin inactivation. Note that the top of the graph marks the moment at which the rate of thrombin formation equals the rate of thrombin inactivation. The thrombin 
generation lag time (TGTlag) is defined as the time span between the onset of the experiment and the time point at which thrombin levels exceed $2 \mathrm{nM}$. The formation of thrombin is initiated by the intrinsic coagulation pathway which becomes activated upon contact with foreign materials. Materials with higher thrombogenicity typically display shorter TGTlag in comparison with materials with lower thrombogenicity.

\section{Platelet adbesion assays}

\section{Lactate Dehydrogenase (LDH) assay}

At the start of each experiment $200 \mu \mathrm{L}$ citrated PRP was added to each clamped catheter tube followed by $1 \mathrm{~h}$ incubation at $37^{\circ} \mathrm{C}$. The samples were rinsed three times with $200 \mu \mathrm{L}$ PBS to remove loosely adhered platelets. The remaining bound platelets were lysed to release LDH by adding $200 \mu \mathrm{L}$ lysis solution ( $0.8 \%$ Triton $\mathrm{X}-100)$ and incubating $1 \mathrm{~h}$ at room temperature. From each catheter sample $50 \mu \mathrm{L}$ lysis solution was transferred in triplo to the wells of a 96 well-plate. Next, $50 \mu \mathrm{L}$ substrate mix, containing a tetrazolium salt, was added to each well, directly followed by incubation in the dark for $30 \mathrm{~min}$ at room temperature. The tetrazolium salt is converted by $\mathrm{LDH}$ into a red formazan product. Finally, $50 \mu \mathrm{L}$ stop solution was added to each well and the optical density was measured at $490 \mathrm{~nm}$. Platelets, adhered to each catheter tube, were quantified by a standard curve plotted from samples containing known amounts of platelets. Every catheter group was tested in triplo.

\section{Scanning Electron Microscopy (SEM)}

Citrated PRP $(200 \mu \mathrm{L})$ was added to each clamped catheter tube followed by $1 \mathrm{~h}$ incubation at $37^{\circ} \mathrm{C}$. Loosely adhered platelets were removed by rinsing the catheter tubes three times with $200 \mu \mathrm{L}$ PBS. The remaining bound platelets were fixed onto the catheter surfaces by incubating the samples $10 \mathrm{~min}$ in $2.5 \%$ glutaraldehyde, at $4{ }^{\circ} \mathrm{C}$. Samples were dehydrated by $10 \mathrm{~min}$ incubation at room temperature in increasing ethanol concentrations of 50, 70, 80, 95 and 100\%. Then, the samples were freeze dried. From each sample three cylindrical pieces were cut with a length of $0.5 \mathrm{~cm}$. Each of these cylindrical pieces was then cut longitudinally into four equal-sized pieces. This resulted for each catheter group in a total number of twelve catheter pieces for evaluation. The catheter pieces were glued onto a stub, sputter coated with gold and finally analysed. Images were captured at $10 \mathrm{kV}$, with appropriate magnifications. Platelet adhesion was quantified by counting platelets on 2150x magnified SEM-images. Platelet morphology was analysed using Goodman's classification describing platelet morphologies on biomaterial surfaces ${ }^{16}$. Catheter groups were tested in triplo. 


\section{Platelet activation assay}

Lepirudin-anticoagulated PRP $(250 \mu \mathrm{L})$ was added to each clamped catheter tube and incubated $1 \mathrm{~h}$ at $37^{\circ} \mathrm{C}$. Following incubation, $250 \mu \mathrm{L}$ PRP was sampled from each catheter tube and directly anticoagulated with $30 \mu \mathrm{L}$ CTAD. Identically, a $250 \mu \mathrm{L}$ PRP sample was taken at the start of the experiment, to determine baseline platelet activation. Further processing of the PRP samples and quantification of platelet activation, was performed using the Asserachrom ${ }^{\circledR} \beta$-TG ELISA according to the instructions of the manufacturer. Every catheter group was tested in triplo.

\section{Antibacterial, thrombogenic and platelet activating effects of solute $\mathrm{Ag}^{+}$}

To examine possible effects of dissolved $\mathrm{Ag}^{+}$on the investigated parameters, which could appear via silver elution from the nanoparticles in our coatings, the above experiments were slightly modified. A series of increasing $\mathrm{Ag}^{+}$concentrations (0,1, 10 and $100 \mathrm{nM}, 1,10$ and $100 \mu \mathrm{M}$ and 1, 3 and $10 \mathrm{mM}$; added as silver nitrate) was evaluated with respect to antibacterial, thrombogenic and platelet activating effects. These experiments were performed with Sil-PVP catheters. The antimicrobial experiment was modified solely by performing the final incubation step (4 h) in $0.9 \% \mathrm{NaCl}$ with added $\mathrm{Ag}^{+}$. Experiments were done in triplo for each $\mathrm{Ag}^{+}$concentration. The thrombin generation assay and the platelet activation assay were modified solely by the use of PRP with added $\mathrm{Ag}^{+}$ (added as silver nitrate). Each $\mathrm{Ag}^{+}$concentration was evaluated in six-fold and in three-fold with respect to thrombin generation and platelet activation respectively.

\section{Silver elution test}

The amount of silver eluted from silver nanoparticle coatings throughout the experiments was determined for both the antimicrobial assay and the thrombin generation assay. For the antimicrobial assay, the experiment was repeated as described, using unwashed Sil-PVP-Ag(15) catheters. Incubations were, however, performed using $0.9 \% \mathrm{NaCl}$ without bacteria to exclude interference with the silver quantification procedure. The thrombin generation assay was also repeated as described, using unwashed Sil-PVP-Ag(15) catheters. To exclude interference with the silver quantification procedure, incubation was performed in $0.9 \% \mathrm{NaCl}$ instead of PRP. For both experiments, the amount of silver eluted from the catheters was determined using inductively coupled plasma-optical emission spectroscopy (ICP-OES) according to DIN EN ISO 11885, after acidification of the silver solutions with nitric acid to $\mathrm{pH} 1$. Catheters were tested in triplo. 


\section{Statistics}

Statistical analysis was performed using Kruskal-Wallis analysis with Dunn's post test for between group comparisons. Separate analyses were performed for thrombin generation experiments performed with PRP from different donors. A p-value less than 0.05 (two-tailed) was considered significant.

\section{Results \& discussion}

\section{Interaction with bacteria}

Results of our experiments to evaluate the antimicrobial activity of the different test samples against Staphylococcus aureus ATCC 29213 are shown in Figure 3. The colonies originate from living bacteria that were transferred from the catheter surface to the agar in the so-called roll-plate assay ${ }^{12}$.

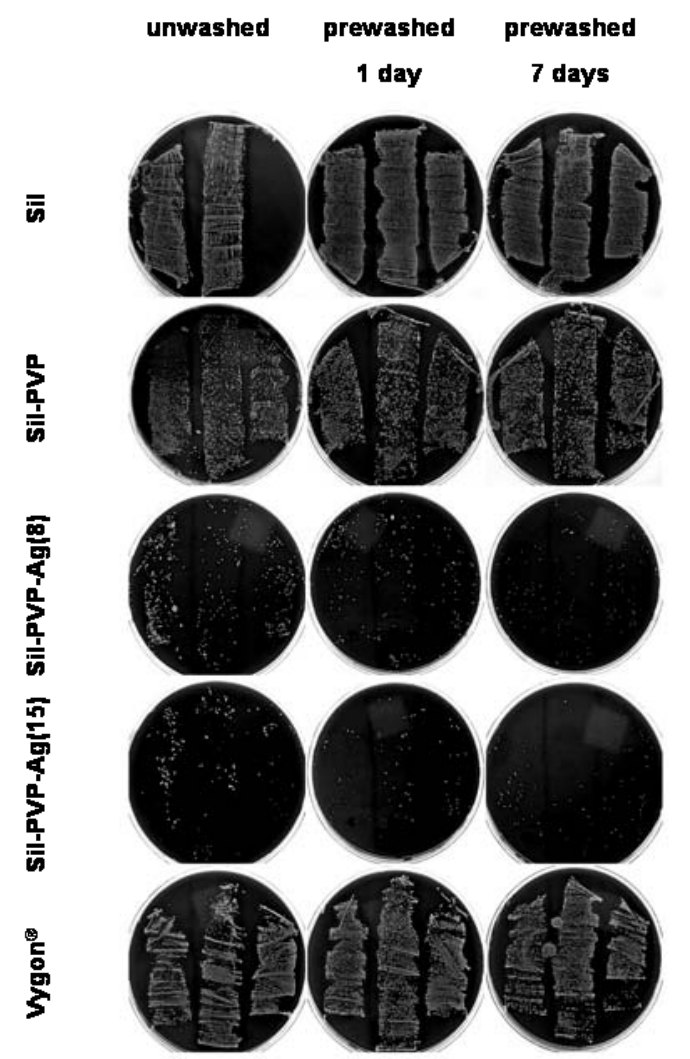

Figure 3. Results of the roll-plate analysis for the catheter groups Sil, Sil-PVP, Sil-PVP-Ag(8), SilPVP-Ag(15) and Vygon®. The figure shows 15 Petri dishes (diameter $10 \mathrm{~cm}$ ) with agar bottom, as well as growing bacterial colonies. Results are shown for catheters in the unwashed state and for catheters incubated 1 or $7 \mathrm{~d}$ in PBS. Experiments were performed in triplo. 
Several conclusions can be drawn from Figure 3. First, there is a clear difference between the number of bacteria that were transferred from the catheters Sil, Sil-PVP and Vygon ${ }^{\circledR}$ on one hand, and the coatings Sil-PVP-Ag $(8)$ and Sil-PVP-Ag(15) on the other hand. This implies that the presence of silver nanoparticles has indeed inhibited bacterial growth. However, none of our agar plates were completely devoid of bacteria. Interestingly, the Sil-PVP-Ag(15) coating did not perform differently from the Sil-PVP-Ag(8) coating. Secondly, we observed that the $\operatorname{Vygon} \AA$ coating did not perform any better than Sil-PVP or Sil. This similarity was observed with unwashed catheters, and also with catheters that were pre-washed (either for 1 day or 7 days).

We repeated the experiment, but now only with Sil-PVP catheters, and in such a way that $\mathrm{Ag}^{+}$were titrated into the culture medium (via $\mathrm{AgNO} 3$ ). Figure 4 shows the results of these experiments; note that the $\mathrm{Ag}^{+}$concentration was increased from $0 \mathrm{nM}$ to $10 \mathrm{mM}$ in 9 steps. The pictures also reveal the antibiotic action of the $\mathrm{Ag}^{+}$: a clear inhibitory effect is seen if the $\mathrm{Ag}^{+}$concentration exceeds $100 \mathrm{nM}$, and no bacteria are found if the $\mathrm{Ag}^{+}$concentration is $10 \mu \mathrm{M}$ or higher. Interestingly, these data shed some more light on the data of Figure 3. Perhaps, the absence of bacterial growth inhibition that was observed for the

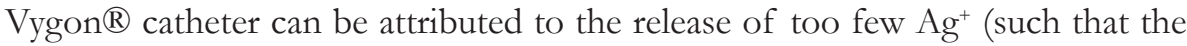
$\mathrm{Ag}^{+}$concentration remains $<100 \mathrm{nM}$ in the interface layer at the catheter's surface). Furthermore, the concentration of $\mathrm{Ag}^{+}$close to the surface of the catheters Sil-PVP-Ag(8) and Sil-PVP-Ag(15) can now be estimated to be in the range $100 \mathrm{nM}-10 \mu \mathrm{M}$. Note that the $\mathrm{Ag}^{+}$concentration in the interface layer at the coating's surface is likely to be much higher than the $\mathrm{Ag}^{+}$concentration in the bulk of the suspension, since there is diffusion limitation, and since the surface coating is the actual depot of the $\mathrm{Ag}^{+}$.
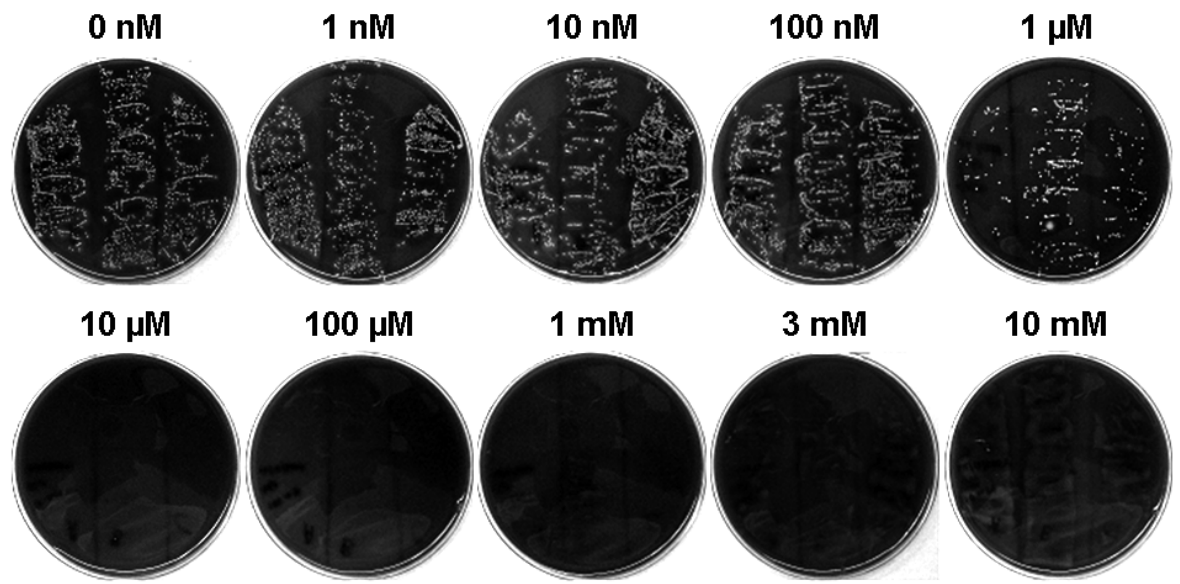

Figure 4. Results of a modified experiment evaluating the antimicrobial properties of $\mathbf{A g}^{+}$in solution without the effect of surface-immobilized silver. Sil-PVP surfaces with adhered bacteria were incubated in the presence of increasing concentrations of $\mathrm{Ag}^{+}$. Next, Sil-PVP catheters were evaluated by roll-plate analysis. Experiments were performed in triplo. 


\section{Interaction with buman PRP - Thrombin generation}

Figure 5 compiles the results of our thrombin generation experiments. Note that the data set represents measurements for the catheters Sil-PVP-Ag(8) and Sil-PVP-Ag(15) and three reference catheters. Moreover, fresh PRP from 5 different donors was used. For each material-donor combination, 6 thrombin generation curves were measured in parallel. Only the TGTlag data are shown. The measurements of TGTlag were found to be nicely reproducible, as is evident from the relatively small standard deviations in the bars in Figure 5.

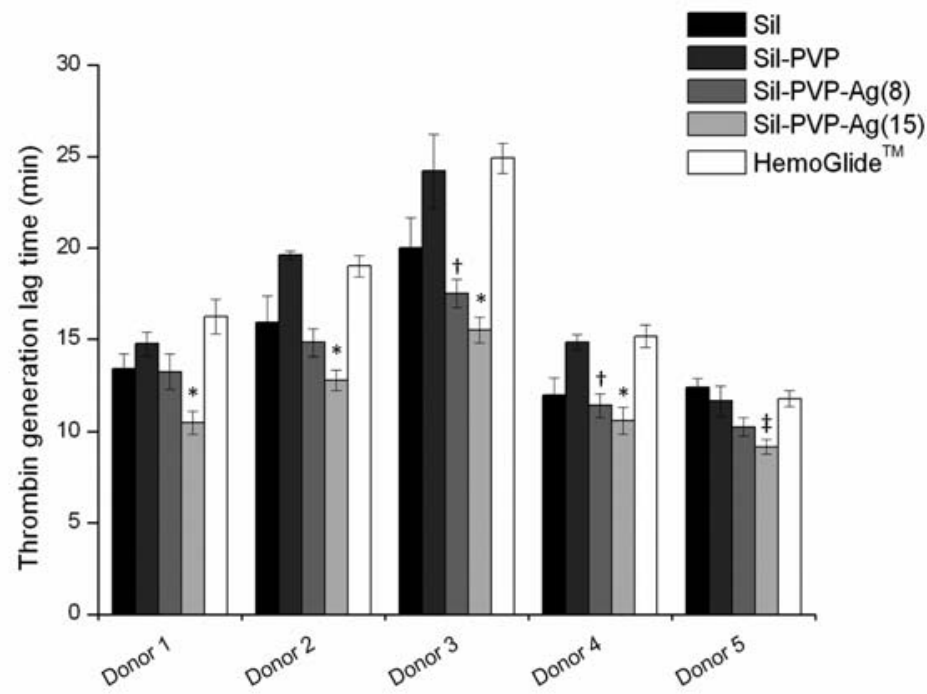

Figure 5. Thrombin generation assays for five different donors. Thrombin generation lag times (TGTlag) are presented for Sil, Sil-PVP, Sil-PVP-Ag(8), Sil-PVP-Ag(15) and HemoGlide ${ }^{\text {TM }}$ catheters. Data are shown as mean \pm standard deviation. Experiments were performed in six-fold. (*) Sil-PVP-Ag(15) TGTlag significantly different compared to Sil-PVP and HemoGlide ${ }^{\mathrm{TM}}$. ( $\ddagger$ ) Sil-PVP-Ag(15) TGTlag significantly different compared to Sil, Sil-PVP and HemoGlide ${ }^{\mathrm{TM}}$. (†) Sil-PVP-Ag(8) TGTlag significantly different compared to HemoGlide ${ }^{\mathrm{TM}}$.

Interestingly, the data in Figure 5 point at several directions. First, there appears to be a substantial donor-variation. Overall, thrombin generation is slowest with the PRP of donor \#3, and fastest with the PRP from donor \#5. Secondly, a consistent pattern in the bar-heights is seen for all five donors. In all cases, TGTlag is shortest for the catheter Sil-PVP-Ag(15). This is clearly evident for the donors \#1, 2, 3 and 5, and to a lesser extent for donor \#4. Thirdly, we see for all donors that TGTlag increases upon going from Sil-PVP-Ag(15) to SilPVP-Ag(8), i.e., when the content of silver nanoparticles in the coating is (approximately) halved. Finally, the data in Figure 5 show that the hydrophilic Sil-PVP performs almost as well as HemoGlide ${ }^{\mathrm{TM}}$. This is in line with several previous reports (from others and from ourselves) stating that hydrophilic coatings have a high level of hemocompatibility ${ }^{17,18}$. 
The data in Figure 5 point at a thrombogenic effect of the silver nanoparticles, which is clearly not desirable. We envisage two possible explanations. Either, released $\mathrm{Ag}^{+}$are responsible for the effect (analogous to the antimicrobial effect, vide supra); it is to be expected that Sil-PVP-Ag(15) releases more $\mathrm{Ag}^{+}$than Sil-PVP-Ag(8) (under identical circumstances). Or, alternatively, the effect is due to activation of blood platelets through collision with silver particles that are exposed at the surface. Evidently, if a platelet contacts the surface of Sil-PVP-Ag(15), the probability to hit silver is approximately twice as high as in the case of Sil-PVP-Ag(8).

In an attempt to discriminate between these two alternative explanations, we again did a titration experiment with $\mathrm{Ag}^{+}$. We used Sil-PVP catheters and PRP from donor \#4, and the $\mathrm{Ag}^{+}$concentration was increased in 9 steps from 0 tot $10 \mathrm{mM}$. Thrombin generation curves were also measured in six-fold. Figure 6 shows the results of this series of experiments. Interestingly, thrombin generation is not affected by the $\mathrm{Ag}^{+}$until a rather high concentration of $100 \mu \mathrm{M}$ is reached. At $1 \mathrm{mM} \mathrm{Ag}$, thrombin generation starts after 4 min already. Thrombin formation is almost immediate if the $\mathrm{Ag}+$ concentration is $3 \mathrm{mM}$ or higher.

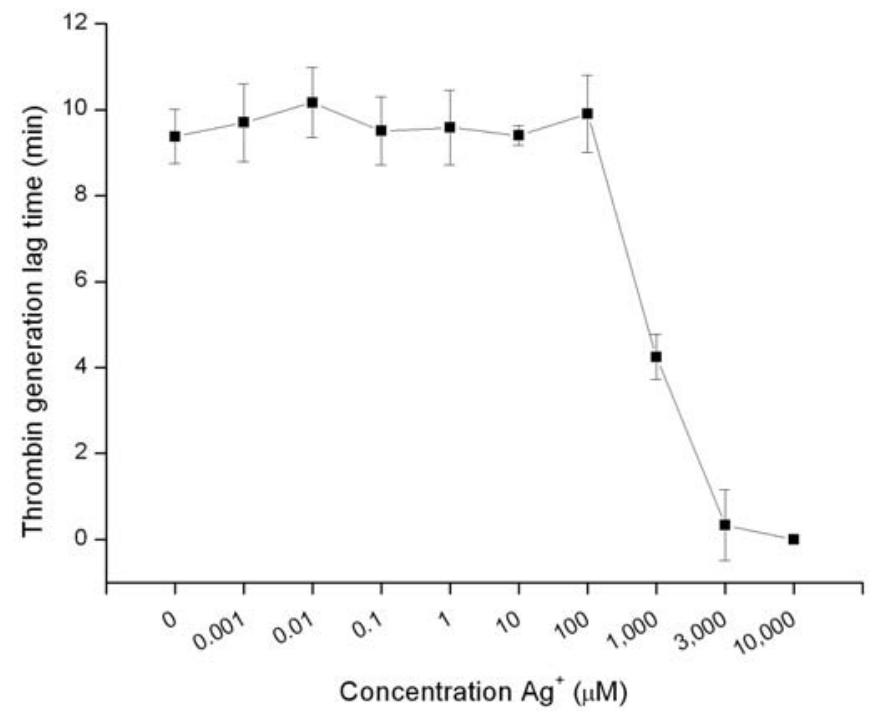

Figure 6. Results of a modified thrombin generation assay evaluating the effect of $\mathrm{Ag}^{+}$in solution on thrombin generation on Sil-PVP catheters, without the effect of surface-immobilized silver. Data are shown as mean \pm standard deviation. Experiments were performed in six-fold.

Our first titration experiment, in which the antimicrobial effect of $\mathrm{Ag}^{+}$ was studied, revealed that the concentration of $\mathrm{Ag}^{+}$in the boundary layer (i.e., at the interface between the catheter's surface and the culture medium or PRP) is 
in the range $100 \mathrm{nM}-10 \mu \mathrm{M}$. Interestingly, this rules out the explanation that release of $\mathrm{Ag}^{+}$from Sil-PVP-Ag(8) and Sil-PVP-Ag(15) is responsible for the observed thrombogenicity: the local concentration of $\mathrm{Ag}^{+}$is in both cases far too low to exert this effect.

Another point is that the experimental conditions during our thrombin generation experiments are such that there is also adsorption of plasma proteins onto the samples. Nevertheless, we observed clear effects due to the presence of silver nanoparticles. This implies that the adsorbed plasma proteins by no means isolate the sample surfaces from the liquid environment.

\section{Interaction with human $P R P$ - Binding and activation of platelets}

Binding of platelets to the different catheters surfaces was first measured with the well-known lactate dehydrogenase assay. Next to the experimental catheters Sil-PVP-Ag(8) and Sil-PVP-Ag(15), the catheters Sil, Sil-PVP, PVC and PVC-Carmeda ${ }^{\circledR}$ were included. The results of these experiments are shown in Figure 7. Although the standard deviations are relatively large, it is clear that the PVC surface attracts most platelets. Few platelets (around $2000 \mathrm{~cm}-2$ ) are found on both Sil-PVP-Ag(8) and Sil-PVP-Ag(15).

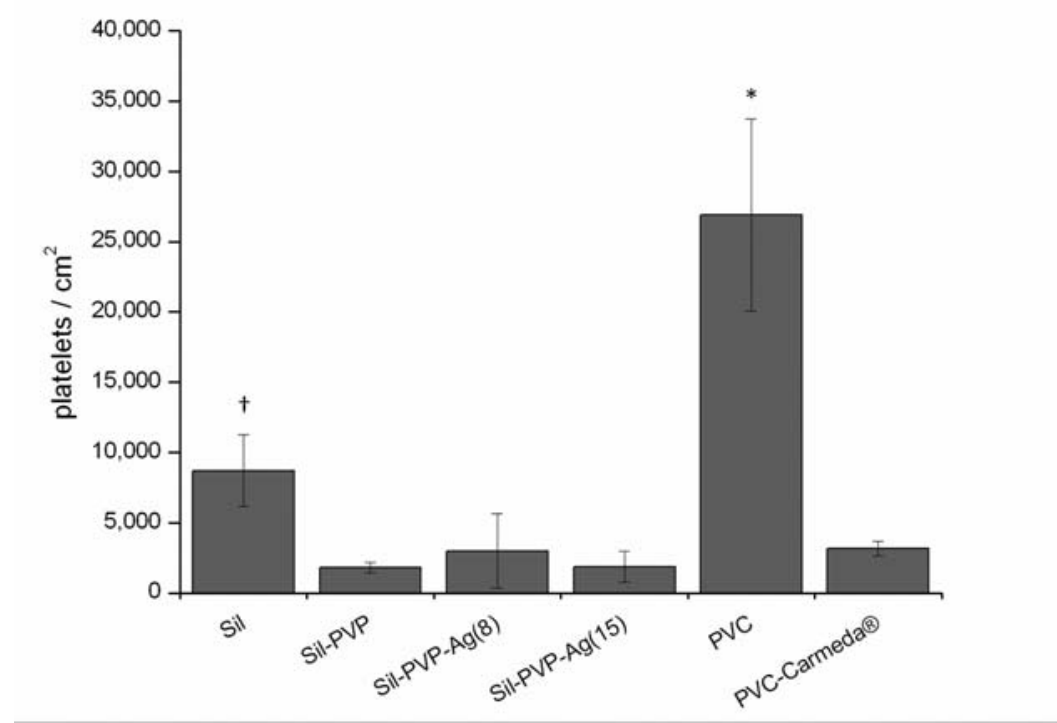

Figure 7. Levels of platelet adhesion onto Sil, Sil-PVP, Sil-PVP-Ag(8), Sil-PVP-Ag(15), PVC and PVCCarmeda ${ }^{\circledR}$ catheters. Data are shown as mean \pm standard deviation. Experiments were performed in triplo. (*) Platelets $/ \mathrm{cm}^{2}$ significantly different compared to Sil-PVP, Sil-PVP-Ag(8) and Sil-PVP-Ag(15). (†) Platelets $/ \mathrm{cm}^{2}$ significantly different compared to Sil-PVP and Sil-PVP-Ag(15).

Secondly, we studied platelet adhesion with scanning electron microscopy. This technique confirmed the data of the LDH assay, in the sense that only few 
adhered platelets could be found on the catheters Sil-PVP, Sil-PVP-Ag(8), SilPVP-Ag(15) and PVC-Carmeda ${ }^{\circledR}$. Figure 8 shows SEM micrographs of platelets adhered to the Sil catheter (A) and to the PVC catheter (B). Tables 2 and 3 compile the data derived from the SEM micrographs. Note that Table 3 provides further information on the morphology of the surface-bound platelets.

a

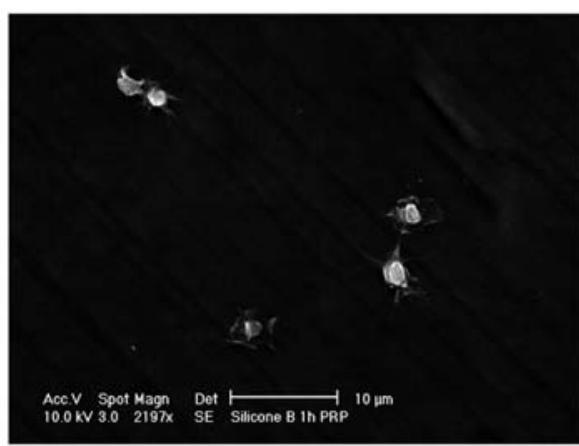

b

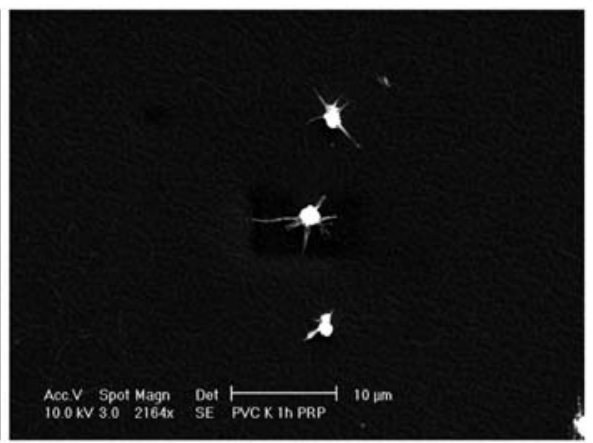

Figure 8. (a) Platelets adhered to a Sil catheter. The platelets have a spread dendritic morphology. (b) Dendritic platelets adhered to a PVC catheter.

Table 2. Results of the SEM analysis with respect to platelet adhesion for the Sil, Sil-PVP, Sil-PVPAg(8), Sil-PVP-Ag(15), PVC and PVC-Carmeda ${ }^{\circledR}$ catheters. Summarized are the qualitative analyses of all twelve catheter pieces evaluated for each catheter group.

\begin{tabular}{|l|c|c|c|c|c|c|c|c|c|c|c|c|c|}
\hline Platelet adhesion & A & B & C & D & E & F & G & H & I & J & K & L & Total \\
\hline Sil & 1 & 8 & 14 & 1 & 0 & 8 & 4 & 5 & - & 2 & 2 & 1 & 46 \\
\hline Sil-PVP & 1 & 0 & 0 & 0 & 0 & 0 & 0 & 0 & 1 & 1 & 0 & 0 & 3 \\
\hline Sil-PVP-Ag(8) & 0 & 0 & 0 & 0 & 0 & 0 & 0 & 0 & 0 & 0 & 0 & 0 & 0 \\
\hline Sil-PVP-Ag(15) & 0 & 0 & 0 & 2 & 0 & 0 & 0 & 0 & 0 & 0 & 0 & 0 & 2 \\
\hline PVC & 1 & 4 & 5 & 8 & 9 & 5 & 2 & 3 & 4 & 2 & 6 & 0 & 49 \\
\hline PVC-Carmeda $\mathbb{P}$ & 0 & 0 & 0 & 0 & 0 & 4 & 0 & 0 & 0 & 3 & 0 & 0 & 7 \\
\hline
\end{tabular}

Table 3. Results of the SEM analysis with respect to platelet morphology for the Sil, Sil-PVP, Sil-PVPAg(8), Sil-PVP-Ag(15), PVC and PVC-Carmeda ${ }^{\circledR}$ catheters. Platelet morphology was analysed using Goodman's classification describing increasingly spread platelet morphologies onto biomaterial surfaces: round (R), dendritic (D), spread dendritic (SD), spread (S) and fully spread (FS) ${ }^{16}$. Summarized are the results for all twelve catheter pieces evaluated for each catheter group.

\begin{tabular}{|l|c|c|c|c|c|}
\hline Platelet morphology & R & D & SD & S & FS \\
\hline Sil & $10 / 46$ & $20 / 46$ & $14 / 46$ & $2 / 46$ & $0 / 46$ \\
\hline Sil-PVP & $2 / 3$ & $1 / 3$ & $0 / 3$ & $0 / 3$ & $0 / 3$ \\
\hline Sil-PVP-Ag(8) & $0 / 0$ & $0 / 0$ & $0 / 0$ & $0 / 0$ & $0 / 0$ \\
\hline Sil-PVP-Ag(15) & $0 / 2$ & $2 / 2$ & $0 / 2$ & $0 / 2$ & $0 / 2$ \\
\hline PVC & $15 / 49$ & $31 / 49$ & $2 / 49$ & $1 / 49$ & $0 / 49$
\end{tabular}


Thirdly, we measured the state of activation of the platelets, using the well-known $\beta$-thromboglobulin assay ${ }^{19}$. These results are shown in Figure 9. A strong activating effect of our experimental catheters Sil-PVP-Ag(8) and SilPVP-Ag(15) is noted; the differences with all controls are quite striking. The $\beta$-thromboglobulin levels remain close to the base line for all control surfaces. Again, we performed a titration experiment. $\mathrm{Ag}^{+}$were added to PRP in such a way that the concentration increased from 0 to $10 \mathrm{mM}$ in 9 steps. Subsequently, levels of $\beta$-thromboglobulin were measured. Figure 10 shows the results. Apparently, the presence of $\mathrm{Ag}^{+}$, up to a concentration of $100 \mathrm{mM}$, has no effect on platelet activation. For higher $\mathrm{Ag}^{+}$concentrations $(>1 \mathrm{mM})$ a pronounced effect was observed.

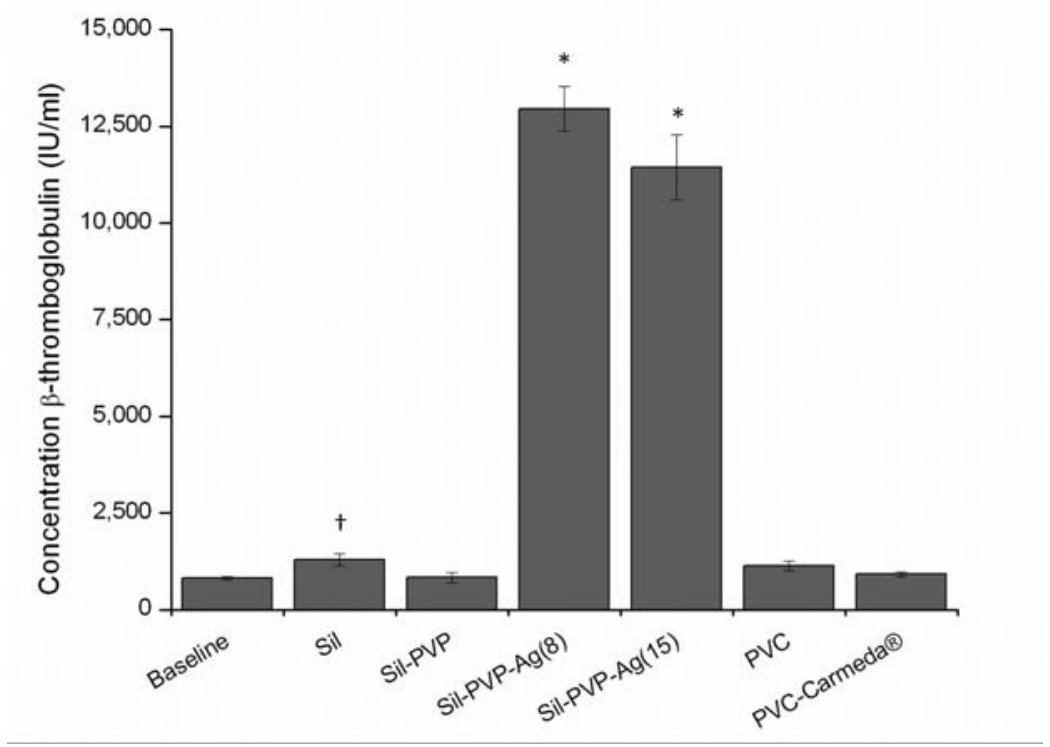

Figure 9. Baseline platelet activation at the start of the experiment and platelet activation after incubation of platelet-rich plasma (PRP) in Sil, Sil-PVP, Sil-PVP-Ag(8), Sil-PVP-Ag(15), PVC and PVC-Carmeda ${ }^{2}$ catheters. Data are shown as mean \pm standard deviation. Experiments were performed in triplo. $\left(^{*}\right)$ Concentration $\beta$-thromboglobulin significantly different compared to baseline, Sil-PVP and PVC-Carmeda ${ }^{\circledR}$. ( $†$ ) Concentration $\beta$-thromboglobulin significantly different compared to baseline and Sil-PVP. 


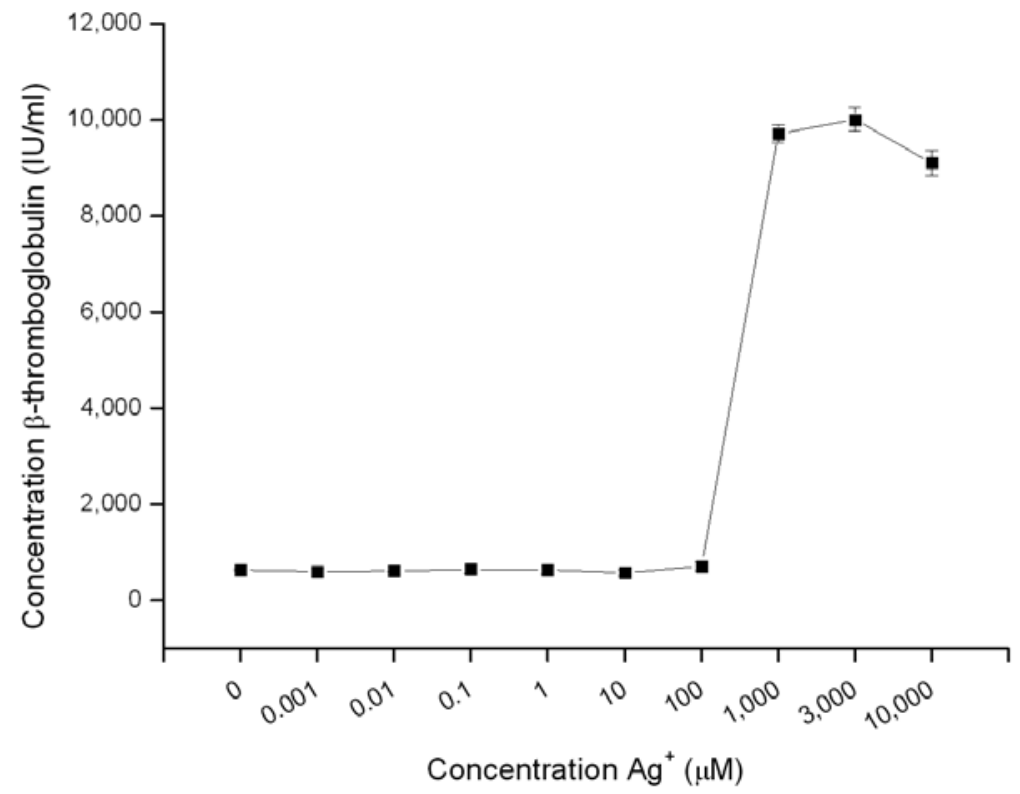

Figure 10. Results of a modified platelet activation assay evaluating the effect of $\mathrm{Ag}^{+}$in solution on platelet activation. Data are shown as mean \pm standard deviation. Experiments were performed in triplo.

\section{Contact-dependent effects of silver nanoparticles}

The exact mechanism behind the antimicrobial action of silver nanoparticles is complex and still a matter of controversy. After adhesion, bacteria on silver nanoparticle coatings probably encounter high amounts of antimicrobial $\mathrm{Ag}^{+}$resulting in their death. Our data suggest that blood platelets become activated upon collision with surface-exposed silver nanoparticles, as shown in Figure 11. It is well known that silver nanoparticles contain both metallic silver $\left(\mathrm{Ag}^{0}\right)$ and $\mathrm{Ag}^{+}{ }^{20,21}$. The content of $\mathrm{Ag}^{+}$is approximately $12 \%$, and most of the ions reside at the periphery of each particle 22. Therefore, we suggest that contact of the platelet's exterior membrane with a silver nanoparticle during a collision leads to immediate and synchronous transfer of $\mathrm{Ag}^{+}$from the particle to the outer phospholipid membrane sheet of the platelet. Note that this mechanism proposes direct (i.e. solvent-free) transfer of $\mathrm{Ag}^{+}$. Then, after desorption of the platelet, the membrane-bound $\mathrm{Ag}^{+}$may induce activation. In turn, activated platelets enhance thrombin formation and activation of more platelets, a process that accelerates itself. 
a
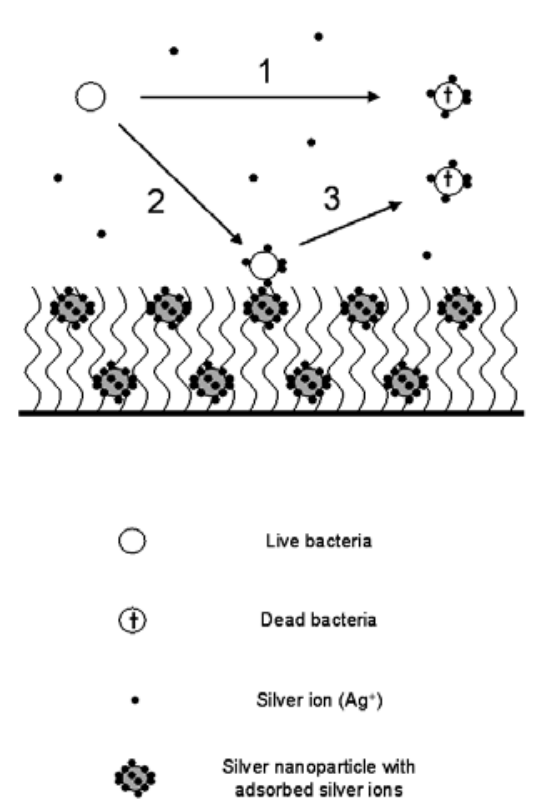

b
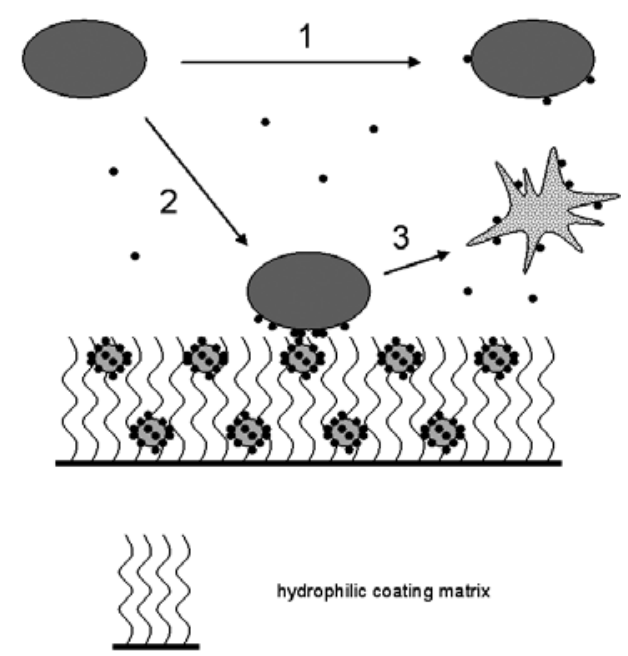

hydrophilic coating matrix

Resting blood platelet

Activated blood platelet

Figure 11. Schematic representation of the observed antimicrobial and platelet activating effects. Panel a: 1. Concentrations of $\mathrm{Ag}^{+}$built up by elution from the silver nanoparticle coating were high enough to kill bacteria. 2 and 3: Possible killing of bacteria by contact-dependent transfer of $\mathrm{Ag}^{+}$following collision with the silver nanoparticle-containing surface. Panel b: 1 . Elution of $\mathrm{Ag}^{+}$from the silver nanoparticle coating did not result in $\mathrm{Ag}^{+}$concentrations high enough to activate blood platelets. 2 and 3: Activation of blood platelets by contact-dependent transfer of $\mathrm{Ag}^{+}$following collision with the silver nanoparticle-containing surface.

Two further remarks can be made: (i), to the best of our knowledge, there are no reports on activation of platelets via $\mathrm{Ag}^{+}$and (ii), direct transfer of $\mathrm{Ag}^{+}$ from silver nanoparticles has been suggested previously, e.g., by Lok, et al. in their studies on the mechanism by which silver nanoparticles deliver $\mathrm{Ag}^{+}$to bacteria ${ }^{22}$. Whether the observed antimicrobial effects are caused by high concentrations of eluted $\mathrm{Ag}^{+}$or by the proposed collision model remains unanswered. Possibly the observed antimicrobial effect is a combination of both mechanisms (Figure 11). We realize that the mechanisms underlying the observed antimicrobial and thrombotic effects may be much more complex in an in vivo setting.

Biomaterial surface properties, partly determined by the exposure of silver nanoparticles, may affect when and which plasma proteins will adsorb, and how their conformation will be changed upon adsorption ${ }^{23,24,25}$. A protein layer could influence antimicrobial properties. On one hand, it could hamper elution of silver from the coating, increasing the time required to reach silver concentrations high enough for antimicrobial activity. On the other hand, such a protein layer could form a barrier preventing direct contact between the 
bacteria and the coating surface. In vivo, the outer catheter surfaces contact flowing blood, resulting in an instant wash-away of eluted $\mathrm{Ag}^{+}$. A flow situation thus prevents build-up of antimicrobial $\mathrm{Ag}^{+}$concentrations, underlining the importance of contact-dependent mechanisms of antimicrobial action in preventing device-related bloodstream infections.

Coagulation is believed to occur faster in non-flowing blood ${ }^{26}$. Our results suggest contact-dependent platelet-activating effects of silver nanoparticle coatings. Whether a flow situation would decrease platelet activation via collision is an interesting subject for further experimentation. On one hand, flow reduces the time-of-contact between platelets and the catheter surface. On the other hand, however, a greater number of platelets will contact the catheter surfaces under flow.

\section{Conclusion}

The most important conclusion that can be drawn from the present data is that the use of silver nanoparticles to impart antibiotic features into surface coatings can compromise the blood-compatibility. This is particularly relevant for indwelling catheters which are used in prolonged contact with the blood of critically ill patients, such as CVCs. Silver nanoparticles appear to provide an adequate solution for one of the two problems that are associated with the use of CVCs, i.e. bacterial line infection. However, the approach may lead to an enhanced second problem, i.e. catheter-associated thrombus formation. This new insight may stimulate further research and development on surface coatings that effectively combine antimicrobial features on one hand, and close-to-zero thrombogenicity on the other hand.

\section{Acknowledgements}

The authors would like to thank Mr. Stefan G.J.A. Camps and Mrs. Christel Driessen for their assistance during the laboratory experiments.

This study was financed within the framework of the Bioterials program, a 4-years private-public joint effort of the Dutch Ministry of Economic Affairs, the Province of Dutch Limburg, DSM Research B.V. (Geleen, The Netherlands), the Academic Hospital Maastricht, and the University of Maastricht. Bioterials runs in the period April 2005 - April 2009. This study was also supported by the Deutschen Forschungsgemeinschaft through the Graduiertenkolleg "Biointerface - Detektion und Steuerung grenzflächeninduzierter, biomolekularer und zellulärer Funktionen" (GRK 1035/2). The Universities of Aachen, Liège and Maastricht cooperate within the framework of this Graduiertenkolleg. 


\section{References}

1 Williams, D. Defining nanotechnology. Med Device Technol 19, 8, 10 (2008).

2 Furno, F. et al. Silver nanoparticles and polymeric medical devices: a new approach to prevention of infection? J Antimicrob Chemother 54, 1019-1024, (2004).

3 Roe, D., Karandikar, B., Bonn-Savage, N., Gibbins, B. \& Roullet, J. B. Antimicrobial surface functionalization of plastic catheters by silver nanoparticles. I Antimicrob Chemother 61, 869-876, (2008).

4 Chen, X. \& Schluesener, H. J. Nanosilver: a nanoproduct in medical application. Toxicol Lett 176, 1-12, (2008).

5 Wenzel, R. P. \& Edmond, M. B. The impact of hospital-acquired bloodstream infections. Emerg Infect Dis 7, 174-177 (2001).

6 Acedo Sanchez, J. D., Batlle, J. F. \& Feijoo, J. B. Catheter-related thrombosis: a critical review. Support Cancer Ther 4, 145-151, (2007).

7 Ruschulte, H. et al. Prevention of central venous catheter related infections with chlorhexidine gluconate impregnated wound dressings: a randomized controlled trial. Ann Hematol 88, 267-272, (2009).

8 Wenzel, R. P. \& Edmond, M. B. Team-based prevention of catheter-related infections. $N$ Engl J Med 355, 2781-2783, (2006).

9 National Nosocomial Infections Surveillance (NNIS) System Report, data summary from January 1992 through June 2004, issued October 2004. Am J Infect Control 32, 470-485, (2004).

10 Dias, A. A. et al. Hydrophilic coating comprising a polyelectrolyte. (2007).

11 Jin, Y. et al. Controlled nucleation and growth of surface-confined gold nanoparticles on a (3-aminopropyl)trimethoxysilane-modified glass slide: a strategy for SPR substrates. Anal Chem 73, 2843-2849 (2001).

12 Maki, D. G., Weise, C. E. \& Sarafin, H. W. A semiquantitative culture method for identifying intravenous-catheter-related infection. N Engl J Med 296, 1305-1309, (1977).

13 Kopp, R. et al. Effect of hirudin versus heparin on hemocompatibility of blood contacting biomaterials: an in vitro study. Int J Artif Organs 28, 1272-1277 (2005).

14 Wielders, S. J., Ungethum, L., Reutelingsperger, C. P., Bevers, E. M. \& Lindhout, T. Factor Xadriven thrombin generation in plasma: dependency on the aminophospholipid density of membranes and inhibition by phospholipid-binding proteins. Thromb Haemost 98, 1056-1062, (2007).

15 Hemker, H. C. et al. The calibrated automated thrombogram (CAT): a universal routine test for hyper- and hypocoagulability. Pathophysiol Haemost Thromb 32, 249-253, (2002).

16 Goodman, S. L. Sheep, pig, and human platelet-material interactions with model cardiovascular biomaterials. J Biomed Mater Res 45, 240-250, (1999).

17 Hanssen, H. H. et al. Metallic wires with an adherent lubricious and blood-compatible polymeric coating and their use in the manufacture of novel slippery-when-wet guidewires: possible applications related to controlled local drug delivery. $J$ Biomed Mater Res 48, 820-828, (1999).

18 Wu, Y., Simonovsky, F. I., Ratner, B. D. \& Horbett, T. A. The role of adsorbed fibrinogen in platelet adhesion to polyurethane surfaces: a comparison of surface hydrophobicity, protein adsorption, monoclonal antibody binding, and platelet adhesion. J Biomed Mater Res A 74, 722-738, (2005).

19 Ohkawa, R. et al. Platelet release of beta-thromboglobulin and platelet factor 4 and serotonin in plasma samples. Clin Biochem 38, 1023-1026, (2005).

20 Kumar, A., Vemula, P. K., Ajayan, P. M. \& John, G. Silver-nanoparticle-embedded antimicrobial paints based on vegetable oil. Nat Mater 7, 236-241, (2008).

21 Morones, J. R. et al. The bactericidal effect of silver nanoparticles. Nanotechnology 16, 2346-2353, (2005). 
Chapter 3

22 Lok, C. N. et al. Silver nanoparticles: partial oxidation and antibacterial activities. J Biol Inorg Chem 12, 527-534, (2007).

23 Chiumiento, A., Lamponi, S. \& Barbucci, R. Role of fibrinogen conformation in platelet activation. Biomacromolecules 8, 523-531, (2007).

24 Koh, L. B., Rodriguez, I. \& Zhou, J. Platelet adhesion studies on nanostructured poly(lacticco-glycolic-acid)-carbon nanotube composite. J Biomed Mater Res A 86, 394-401, (2008).

$25 \mathrm{Xu}$, L. C. \& Siedlecki, C. A. Effects of surface wettability and contact time on protein adhesion to biomaterial surfaces. Biomaterials 28, 3273-3283, (2007).

26 Lowe, G. D. Virchow's triad revisited: abnormal flow. Pathophysiol Haemost Thromb 33, 455-457, (2003). 
Nanosilver coatings: relationship between antimicrobial effect and blood coagulation 
Disruption and activation of blood platelets in contact with an antimicrobial composite coating consisting of a pyridinium polymer and silverbromide $(\mathrm{AgBr})$ nanoparticles

Stevens $\mathrm{KN}^{1}$, Knetsch $\mathrm{ML}^{2}$, Sen $\mathrm{A}^{3}$, Sambhy $\mathrm{V}^{3}$, Koole $\mathrm{LH}^{2}$.

1. Department of Cardiothoracic Surgery, Maastricht University Medical Centre, Maastricht, The Netherlands.

2. Centre for Biomaterials Research, University of Maastricht, Maastricht, The Netherlands.

3. Department of Chemistry, The Pennsylvania State University, Pennsylvania (PA), USA.

ACS Appl Mater Interfaces. 2009;1(9):2049-54. 


\section{Abstract}

Composite materials made up from a pyridinium polymer matrix and silverbromide nanoparticles embedded therein feature excellent antimicrobial properties. Most probably, the antimicrobial activity is related to the membrane disrupting effect of both the polymer matrix and $\mathrm{Ag}^{+}$; both may work synergistically. One of the most important applications of antimicrobial materials would be their use as surface coatings for percutaneous (skinpenetrating) catheters, such as central venous catheters (CVCs). These are commonly used in critical care, and serious complications due to bacterial infection occur frequently. This study aimed at examining possible effects of a highly antimicrobial pyridinium polymer $/ \mathrm{AgBr}$ composite on the blood coagulation system, i.e., (i), the coagulation cascade leading to the formation of thrombin and a fibrin crosslinked network, and (ii), on blood platelets. Evidently, pyridinium/AgBr composites could not qualify as coatings for CVCs if they trigger blood coagulation. Using a highly antimicrobial composite of NPVP [poly(4-vinylpyridine)-co-poly(4-vinyl-N-hexylpyridinium bromide)] and $\mathrm{AgBr}$ nanoparticles, as a thin adherent surface coating on Tygon $\AA$ elastomer tubes, it was found that contacting blood platelets rapidly acquire a highly activated state, after which they become substantially disrupted. This implies that NPVP/AgBr is by no means blood-compatible. This disqualifies the material for use as a CVC coating. This information, combined with earlier findings on hemolytic effects (i.e. disruption of contacting red blood cells) of similar materials, implies that this class of antimicrobial materials affect not only bacteria, but mammalian cells as well. This would render them more useful outside the biomedical field. 


\section{Introduction}

Recently, it was discovered that composite materials consisting of a cationic pyridinium polymer matrix and $\mathrm{AgBr}$ nanoparticles embedded therein, have pronounced antibacterial properties ${ }^{1}$. This observation was primarily made with the copolymer NPVP [poly(4-vinylpyridine)-co-poly(4-vinyl-Nhexylpyridinium bromide)]. Once placed on surfaces that were overgrown by bacteria (either gram-positive or gram-negative), the NPVP/AgBr composites killed all bacteria under and around them. Furthermore, the antibacterial effect lasted for relatively long periods, i.e. up to several days or weeks ${ }^{1}$. Many parameters could be controlled (e.g. size of the $\mathrm{AgBr}$ nanoparticles, content of $\mathrm{AgBr}$ in the material, and release kinetics of $\left.\mathrm{Ag}^{+}\right)$. It was postulated that the mechanism of action is disruption of the bacterial membrane (initially), followed by sustained release of $\mathrm{Ag}^{+}$, even if the surface is covered with dead bacteria or biofilm ${ }^{1}$.

There is a clear need for antimicrobial materials and coatings in many different fields of application. We are convinced that one of these applications would relate to percutaneous (skin-penetrating) catheters, especially so-called central venous catheters (CVCs). Such catheters are used extensively in the care of critical patients, both for monitoring and therapy. The use of CVCs is, however, associated with two significant problems: one is bacterial colonization, and the other is blood coagulation ${ }^{2,3,4}$. The adherence of bacteria onto the surface of CVCs may lead to bloodstream infections which are, in turn, associated with substantial morbidity, mortality, prolonged hospital stay, and increased cost. Recently, it was reported that the risk for bloodstream infection that is associated with the most common type of CVC (the non-cuffed type) is as high as $2-7$ episodes per 1000 catheter days ${ }^{5}$. Coagulation, initiated by the part of the CVC that is in contact with blood, can lead to formation of thrombotic particulate emboli. When these detach from the CVC's surface, they can cause occlusion of relatively larger vital arteries in the lungs or in the brain (stroke), which may have detrimental consequences ${ }^{3}$.

Clearly, NPVP/AgBr composites would not qualify as CVC coatings despite their excellent antimicrobial properties- if the cationic polymer and/or the $\mathrm{AgBr}$ nanoparticles would result in a hemolytic and/or thrombogenic surface. With respect to hemolysis, it has been reported already that positively charged pyridinium polymers can lead to disruption of contacting red blood cells (hemolysis), especially when the positive charges at the material's surface are relatively far apart from each other ${ }^{6}$. To the best of our knowledge, the aptitude of pyridinium polymers to initiate coagulation of blood has not been studied. Therefore, we became interested in examining the blood compatibility of NPVP/AgBr composites. Here, we describe an in vitro study on a NPVP/AgBr composite in contact with fresh human platelet-rich plasma (PRP). Blood from six healthy volunteer-donors was used. The NPVP/AgBr composite coating was 


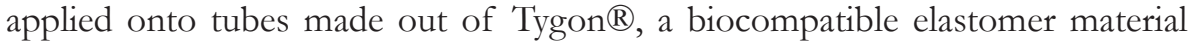
which is applied in many different catheters. Unmodified Tygon $\AA$ tubes, and

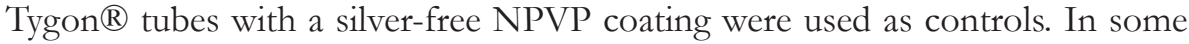
experiments, we also included commercially available CVC catheter materials for referencing. We studied surface-induced thrombin generation, activation of blood platelets due to contact with the surface coating, and morphology of adhered platelets. The results are discussed in the context of the potential utility of NPVP/AgBr composites as coatings for indwelling medical devices that are intended to function for extended periods (up to several weeks).

\section{Materials \& methods}

\section{Materials}

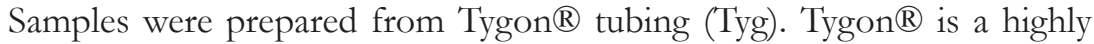
stable polymer material that is used in the catheter industry. NPVP and NPVP/AgBr were synthesized by the Pittsburg group, according to protocols that were published previously ${ }^{1}$. The materials were applied as adherent coatings onto the outer and inner surfaces of pieces of the Tygon ${ }^{\circledR}$ tubing (Tyg-NPVP and Tyg-NPVP-AgBr; length approximately $10 \mathrm{~cm}$ ), by the same group. As controls, we used polyvinylchloride (PVC) tubing of the same dimensions, PVC tubing carrying the PVC-Carmeda ${ }^{\circledR}$ immobilized heparin coating (also of the same dimensions; PVC-Carmeda $\left.{ }^{\circledR}\right)$, and/or the Bard HemoGlide ${ }^{\mathrm{TM}}$ CVC catheter (HemoGlide ${ }^{\mathrm{TM}}$ ) ${ }^{7}$, which is the CVC that is used mostly in different Departments of the Maastricht University Medical Centre+. Table 1 provides a comprehensive overview.

$\mathrm{NaCl}, \mathrm{CaCl} 2(\mathrm{H} 2 \mathrm{O}) 2$, glutaraldehyde 25\%, dimethylsulfoxide (DMSO) and Triton X-100, were purchased from Acros Organics (Geel, Belgium). Na3citrate and K2HPO4 were products from Sigma-Aldrich Chemie B.V. (Zwijndrecht, The Netherlands). KH2PO4 was from Janssen Chimica (Beerse, Belgium) and ethanol 100\% was from Merck KGaA (Darmstadt, Germany). Lepirudin (Refludan) was obtained from Pharmion (Windsor Berkshire, UK). The fluorogenic substrate for thrombin, Z-Gly-Gly-Arg-AMC, was a product of Bachem Holding AG (Bubendorf, Switzerland; ref. I-1140). The plateletstabilizing anticoagulant mixture, citrate-theophylline-adenosine-dipyridamole (CTAD), was purchased from Becton Dickinson (Alphen aan den Rijn, The Netherlands). The lactate dehydrogenase (LDH) assay was performed using the Cyto'Tox 96® Non-Radioactive Cytotoxicity Assay from Promega Benelux B.V. (Leiden, The Netherlands). Platelet activation was quantified with the Asserachrom ${ }^{\circledR} \beta$-TG enzyme-linked immunosorbent assay (ELISA), obtained from Roche Diagnostics Nederland B.V. (Almere, The Netherlands). 
Table 1. Overview of the catheter materials used throughout this study.

\begin{tabular}{|c|c|c|c|c|}
\hline Catheter & $\begin{array}{l}\text { Commercial } \\
\text { source }\end{array}$ & Coating & $\begin{array}{l}\text { Inner Diameter } \\
(\mathrm{mm})\end{array}$ & $\begin{array}{l}\text { Outer Diameter } \\
(\mathrm{mm})\end{array}$ \\
\hline HemoGlide $^{\mathrm{TM}}$ (a) & Yes & No & $\begin{array}{l}2 \text { lumens; } \\
2.3 \mathrm{~mm} \text { each }\end{array}$ & 4.8 \\
\hline PVC-Carmeda ${ }^{\circledR}(b)$ & Yes & 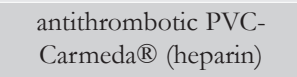 & 4.8 & 7.9 \\
\hline PVC (c) & Yes & No & 4.8 & 7.9 \\
\hline Tyg (d) & Yes & No & 4.8 & 6.4 \\
\hline Tyg-NPVP & No & cationic NPVP coating (e) & 4.8 & 6.4 \\
\hline Tyg-NPVP-AgBr & No & $\begin{array}{l}\text { cationic NPVP coating } \\
\text { containing silver bromide } \\
(\mathrm{AgBr}) \text { nanoparticles }(\mathrm{e})\end{array}$ & 4.8 & 6.4 \\
\hline
\end{tabular}

(a) Purchased from Bard Nordic (Helsingborg, Sweden; ref. 5664190). (b) Purchased from Medtronic Nederland (Heerlen, The Netherlands; ref. M311902A). (c) Purchased from Medos Medizintechnik AG (Stolberg, Germany; ref. 039682). (d) Purchased from Saint-Gobain Performance Plastics (Charny, France; ref. Tygon ${ }^{\circledR}$ S-50-HL AAX00011) (e) Prepared as a part of this study.

\section{Equipment}

Platelets were counted on an automatic cell counter (Coulter AC-T diff, Beckman Coulter, Miami, FL, USA). Fluorescence tracings were recorded on a SpectraMax M2 spectrofluorometer (Molecular Devices, Sunnyvale, CA, USA). Absorbances were measured on an ELx808 Absorbance Microplate Reader (BioTek Instruments, Inc., VT, USA). Sputter coating was performed with a Sputter coater 108 auto/SE (Cressington Scientific Instruments Ltd., Watford, UK) and for scanning electron microscopy (SEM) analysis, a Philips XL30 Scanning Electron Microscope (Philips, Eindhoven, The Netherlands) was used. Centrifugation was performed in a MSE Mistral 3000 I centrifuge (Beun - de Ronde, Abcoude, The Netherlands).

\section{Preparation of the catheter samples}

Catheter samples were cut to the correct dimensions (vide infra) in a laminar flow cabinet. The catheter Tyg was used as a reference in all of our experiments. In most of our experiments we used the catheter samples directly as incubation wells by closing one end with a surgical tube clamp ${ }^{8}$. We also tested commercially available control materials in parallel throughout our experiments. However, the dimensions of these commercial products did not allow them to be used consistently throughout all experiments. The HemoGlide $^{\mathrm{TM}}$ catheter is the central venous catheter of choice in our 
institution. It was used in all of our thrombin generation assays. However, because of its double lumen, it was impossible to use HemoGlide ${ }^{\text {TM }}$ in the other assays. PVC tubing (single lumen), with and without the PVC-Carmeda ${ }^{\circledR}$ coating, was used as a reference material in our LDH assays, in our SEM analyses, and in our platelet activation assay. However, the thickness of the wall precluded the use of these materials in our thrombin generation assay.

\section{Blood collection and preparation of platelet-rich plasma}

Blood was collected through venipuncture from 6 healthy non-smoking male volunteer-donors who did not take any hemostasis-influencing medication at least 10 days before the experiment. Depending on the experiment to be performed, the blood was anticoagulated immediately with either citrate (endconcentration: $0.013 \mathrm{M}$ citrate), or lepirudin, a recombinant form of hirudin (end-concentration: $20 \mu \mathrm{g} / \mathrm{mL}$ lepirudin). Lepirudin is a direct thrombin inhibitor and executes its anticoagulant effect without interfering with platelet function $9,10,11$. Therefore it is the anticoagulant of choice for platelet activation studies. Then, PRP was isolated from the blood through centrifugation $(200 \mathrm{x} \mathrm{g}$, 15 min, room temperature) ${ }^{8,12,13}$. Before the start of each experiment, the concentration of platelets in the PRP was measured with an automatic cell counter.

\section{Platelet adbesion assay}

The CytoTox 96® Non-Radioactive Cytotoxicity Assay measures the number of viable platelets adhered to a specific surface area of a biomaterial via colorimetric quantification of the enzyme lactate dehydrogenase (LDH), released upon cell lysis. The experiment was performed twice with PRP from two different donors (donor \#2 ([platelets] = $418 \times 103 \mu \mathrm{L}-1$ ) and donor \#4 ([platelets $]=446 \times 103 \mu \mathrm{L}-1)$ ). Throughout each experiment all catheter groups were tested in triplo, with identical PRP.

At the start of each experiment $200 \mu \mathrm{L}$ citrated PRP was added to each clamped catheter tube followed by $1 \mathrm{~h}$ incubation at $37^{\circ} \mathrm{C}$. The samples were rinsed three times with $200 \mu \mathrm{L}$ Phosphate-Buffered Saline (PBS) $(10 \mathrm{mM}$ K2HPO4, $10 \mathrm{mM} \mathrm{KH2PO4,} 150 \mathrm{mM} \mathrm{NaCl}$; $\mathrm{pH}$ 7) to remove loosely adhered platelets. The remaining bound platelets were lysed to release $\mathrm{LDH}$ by adding $200 \mu \mathrm{L}$ lysis solution (0.8\% Triton X-100) and incubating $1 \mathrm{~h}$ at room temperature. From each catheter sample $50 \mu \mathrm{L}$ lysis solution was transferred in triplo to the wells of a 96 well-plate. Next, $50 \mu \mathrm{L}$ substrate mix, containing a tetrazolium salt, was added to each well, directly followed by incubation in the dark for $30 \mathrm{~min}$ at room temperature. The tetrazolium salt is converted by LDH 
into a red formazan product. Finally, $50 \mu \mathrm{L}$ stop solution was added to each well and the optical density was measured at $490 \mathrm{~nm}$. Viable platelets, adhered to each catheter tube, were quantified by a standard curve plotted from samples containing known amounts of platelets.

\section{Morphology of adbered platelets}

These experiments were performed with PRP from donor \#4 ([platelets] $=453 \times 103 \mu \mathrm{L}-1)$ in parallel with the platelet adhesion assay (see above). Citrated PRP $(200 \mu \mathrm{L})$ was added to each clamped catheter tube followed by 1 $\mathrm{h}$ incubation at $37^{\circ} \mathrm{C}$. Loosely adhered platelets were removed by rinsing the catheter tubes three times with $200 \mu \mathrm{L}$ PBS. The remaining bound platelets were fixed onto the catheter surfaces by incubating the samples $10 \mathrm{~min}$ in $2.5 \%$ glutaraldehyde, at $4{ }^{\circ} \mathrm{C}$. Samples were dehydrated by $10 \mathrm{~min}$ incubation at room temperature in increasing ethanol concentrations of 50, 70, 80, 95 and 100\%. Then, the samples were freeze dried. From each sample three cylindrical pieces were cut with a length of $5 \mathrm{~mm}$. Each of these cylindrical pieces was then cut longitudinally into four equal-sized pieces. This resulted for each catheter group in a total number of twelve catheter pieces for evaluation. Each catheter piece was glued onto a stub, sputter coated with gold and finally analysed using SEM. Images were captured at $10 \mathrm{kV}$, with magnifications of 1080x and 4320x. For each catheter piece, two SEM images were captured at each magnification, resulting in twenty-four SEM images per magnification per catheter group. Platelet adhesion was quantified by counting platelets on 4320x magnified SEMimages. Platelet morphology was analysed using Goodman's classification describing platelet morphologies on biomaterial surfaces ${ }^{14}$. Catheter groups were tested in triplo.

\section{Platelet activation}

These experiments were performed with PRP from donor \#4 ([platelets] $=402 \times 103 \mu \mathrm{L}-1)$ in parallel with the platelet adhesion and platelet morphology assays (see above). Lepirudin-anticoagulated PRP $(250 \mu \mathrm{L})$ was added to each clamped catheter tube and incubated $1 \mathrm{~h}$ at $37^{\circ} \mathrm{C}$. Following incubation, $250 \mu \mathrm{L}$ PRP was sampled from each catheter tube and directly anticoagulated with $30 \mu \mathrm{L}$ CTAD. Identically, a $250 \mu \mathrm{L}$ PRP sample was taken at the start of the experiment, to determine baseline platelet activation, which could have occurred during the isolation of PRP. Further processing of the PRP samples and quantification of platelet activation, was performed using the Asserachrom ${ }^{\circledR}$ $\beta$-TG ELISA according to the instructions of the manufacturer. Every catheter group was tested in triplo. 


\section{Thrombin generation}

Each thrombin generation experiment was performed with PRP from a single donor. This allowed all catheter groups to be tested with identical PRP. The experiment was performed six times with PRP from six different donors. In each experiment, every catheter group was tested in six-fold. Catheter samples (length: $4 \mathrm{~mm}$ ) were placed horizontally in the wells of a 96 well-plate ${ }^{8}$. Just before the start of the experiment, a fluorogenic substrate for thrombin, Z-GlyGly-Arg-AMC, was added to citrated PRP to a final concentration of $400 \mu \mathrm{M}$. Next, the citrated PRP was "recalcified" by adding $\mathrm{CaCl} 2$ stock solution $(0.5 \mathrm{M}$ $\mathrm{CaCl} 2$ ) to a final concentration of $20 \mathrm{mM} \mathrm{CaCl}$. Upon recalcification, concentrations of $\mathrm{Ca}_{2}{ }^{+}$were restored to physiological levels, and the blood clot mechanism is no longer inhibited. Then, PRP + fluorogenic substrate was instantly distributed over the wells in volumes of $200 \mu \mathrm{L}$. Wells without a catheter sample served as controls. Fluorescence tracings were recorded at $37^{\circ} \mathrm{C}$, every 30 seconds during $1 \mathrm{~h}\left(\lambda_{\mathrm{ex}}=368 \mathrm{~nm}\right.$ and $\lambda_{\mathrm{em}}=460 \mathrm{~nm}$ and $2 \mathrm{~s}$ shaking prior to each measurement) ${ }^{15}$. The fluorescence intensity was converted into nanomolar concentrations thrombin as described earlier by Hemker, et al. ${ }^{16}$. This resulted in a thrombin generation curve for each well, as shown in Figure 1.

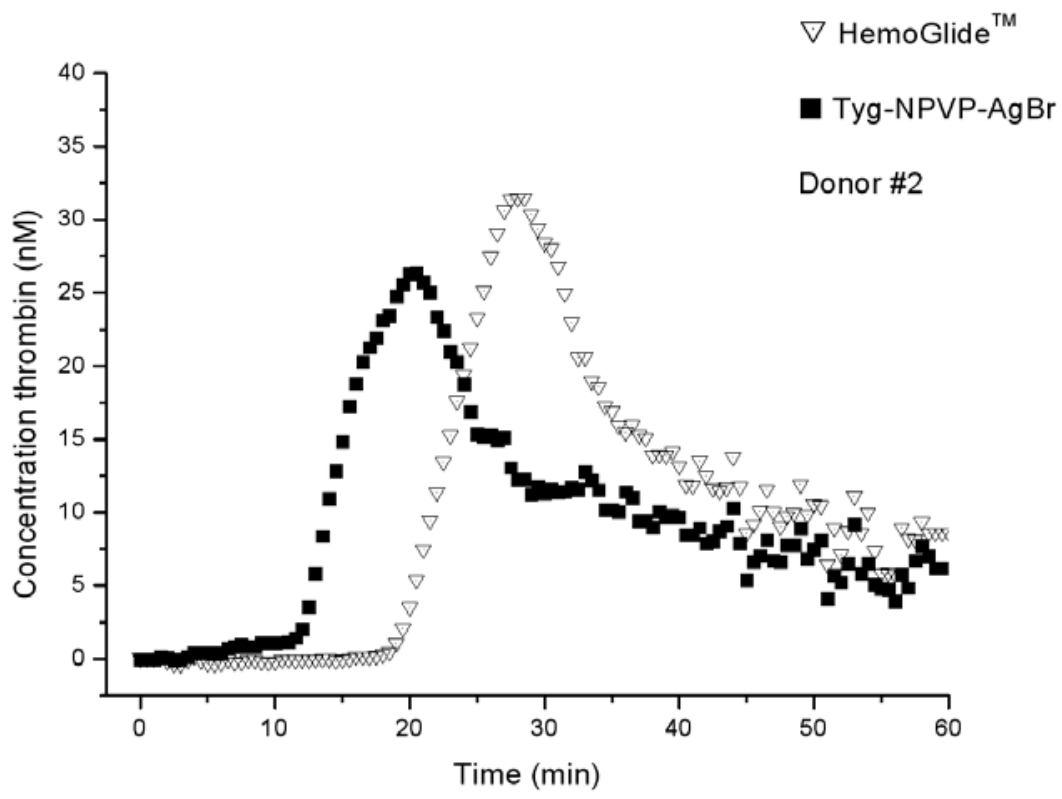

Figure 1. Thrombin generation curves measured in wells with a HemoGlide ${ }^{\mathrm{TM}}$ sample $(\nabla)$ and with a Tyg-NPVP-AgBr sample (ם). Both experiments were performed simultaneously with PRP from a single donor (Donor \#2). Notice how the time span between the onset of the experiment and the sudden rise in thrombin levels differs. 
The thrombin generation assay can be best explained by comparing the control material, HemoGlide ${ }^{\mathrm{TM}}$, and the Tyg-NPVP-AgBr catheter. The control experiment (PRP-substrate- $\mathrm{Ca} 2+$ mixture incubated in presence of the HemoGlide $^{\text {TM }}$ catheter $(\nabla)$ ) shows a typical control thrombin generation curve, which depicts the change of [thrombin] versus time. During the first minutes, [thrombin] remains close to zero. Then, a sudden increase of [thrombin] is seen. The concentration rises steeply, passes a maximum, and slowly decreases back to (approximately) zero. The formation of thrombin is the result of intrinsic coagulation (contact with foreign materials).

In the presence of a Tyg-NPVP-AgBr catheter in the well ( $\mathbf{\square})$, the thrombin generation curve changes, especially in the sense that the time interval between the start of the experiment and the sudden rise of [thrombin] becomes shorter. This time interval, also called the thrombin generation lag time (TGTlag) was measured systematically in this study and was defined as the time span between the onset of the experiment and the time point at which an increase of $\geq 2 \mathrm{nM}$ thrombin is attained between two time points. Materials with lesser hemocompatibility typically display shorter thrombin generation lag times.

\section{Statistics}

Statistical analysis was performed using Kruskal-Wallis analysis with Dunn's post test for between group comparisons. Separate analyses were performed for thrombin generation experiments performed with PRP from different donors. A p-value less than 0.05 (two-tailed) was considered significant.

\section{Results}

\section{Platelet adbesion assay}

These experiments were performed with PRP from two different donors (donors \#2 and \#4); Figure 2a compiles the results for donor \#4. Results were very similar for donor \#2 (data not shown). Clearly, the Tyg-NPVP material has the highest density of adhered viable platelets at the surface (approximately 270,000 per $\mathrm{cm}^{2}$ ). Surprisingly, incorporation of $\mathrm{AgBr}$ nanoparticles in the coating strongly reduces the adsorption of viable platelets to approximately 50,000 per $\mathrm{cm}^{2}$. Similar densities of adhered platelets were found for the other three (control) materials. Figure $2 \mathrm{~b}$ displays the number of platelets counted via SEM, irrespective of their morphology (or viability). 
a

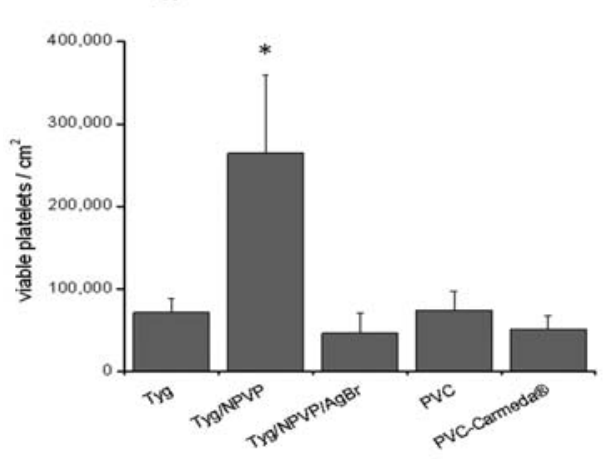

b

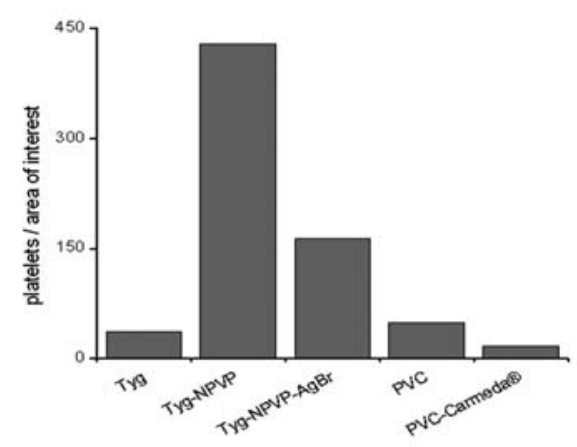

Figure 2. Platelet adhesion onto Tyg, Tyg-NPVP, Tyg-NPVP-AgBr, PVC and PVC-Carmeda ${ }^{\circledR}$ catheters. The graph combines the results from both the LDH assay (a) and SEM analysis (b). During both experiments catheter groups were tested in triplo. Data are shown as mean \pm standard deviation in panel (a) and, in panel (b), as the number of platelets counted on all SEM images for each specific catheter group. $\left(^{*}\right)$ Viable platelets/cm2 significantly different compared to Tyg, Tyg-NPVP-AgBr and PVC-Carmeda®.

\section{Morphology of adbered platelets}

These experiments were done in parallel with the LDH assay (see above), using PRP from donor \#4 and the same biomaterials. Scanning electron microscopy is known to be an excellent technique to study morphology of blood platelets after their adherence to biomaterial surfaces ${ }^{17}$. Figure 3 shows representative SEM micrographs of platelets adhered to the different catheter tubes. In line with the data from the LDH assay, most platelets were found on the Tyg-NPVP surface. To a large extent, the morphologies of these platelets were spread and dendritic with relatively long pseudopodia. An increased surface density of adhered platelets was also seen on the surface of Tyg-NPVP-AgBr samples, compared to Tyg, PVC and PVC-Carmeda ${ }^{\circledR}$ control samples. This differs from the data obtained with the LDH assay (Figure 2a). Additionally, morphologies were remarkably different: most of the platelets adhered to TygNPVP-AgBr appeared dendritic with numerous short pseudopodia, i.e., they were significantly distorted. Apparently, the presence of $\mathrm{AgBr}$ nanoparticles in the coating strongly affects the morphology and viability of contacting blood platelets. 


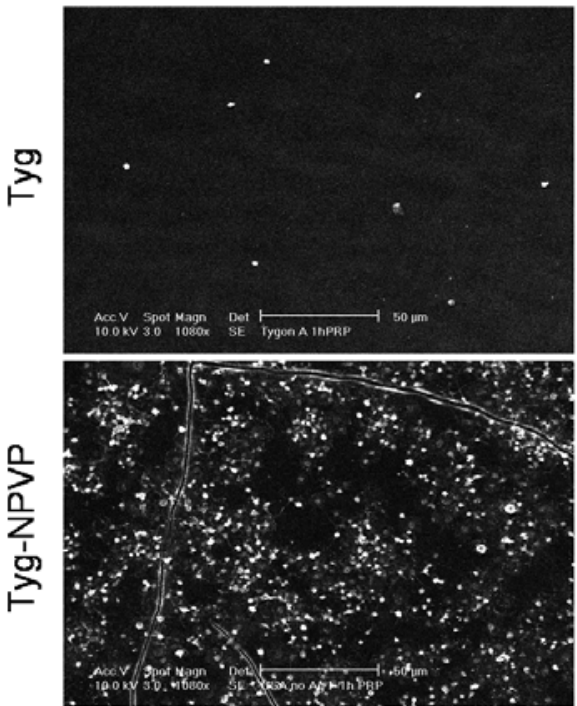

b
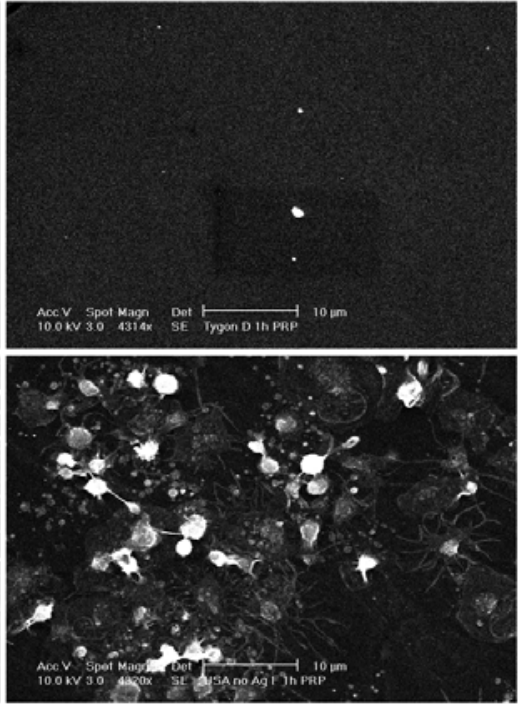

xण

H.

\%.

(5)

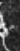

ò

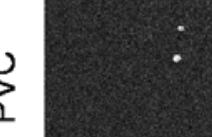

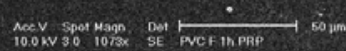

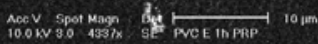

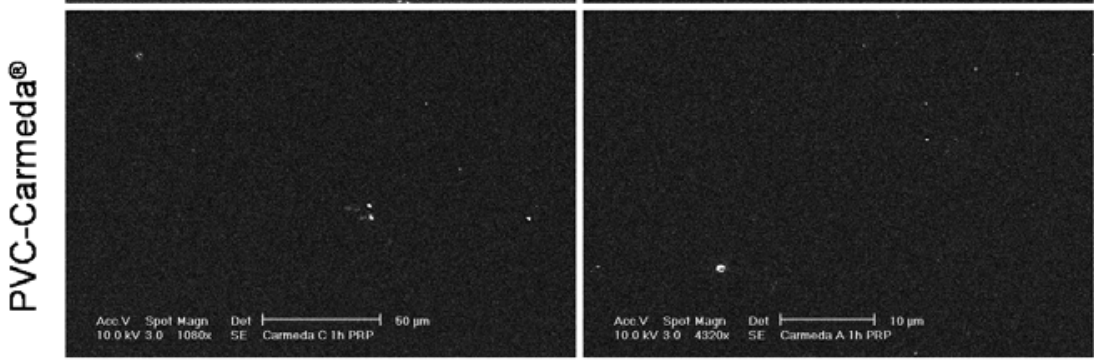

Figure 3. Results of the SEM analysis for Tyg, Tyg-NPVP, Tyg-NPVP-AgBr, PVC and PVCCarmeda ${ }^{\circledR}$ catheters. For each catheter group two representative SEM-images, with magnifications of 1080x (a) and 4320x (b), are shown. 


\section{Platelet activation}

These experiments were done in parallel with the LDH assay and the SEM experiments described above, using PRP from donor \#4 and the same biomaterials. The results are normalized for the number of adhered viable platelets and are shown in Figure 4. Platelets in contact with Tyg, Tyg-NPVP, PVC or PVC-Carmeda ${ }^{\circledR}$ coating do not become activated to a measurable extent: the $\beta$-thromboglobulin levels remain rather low.

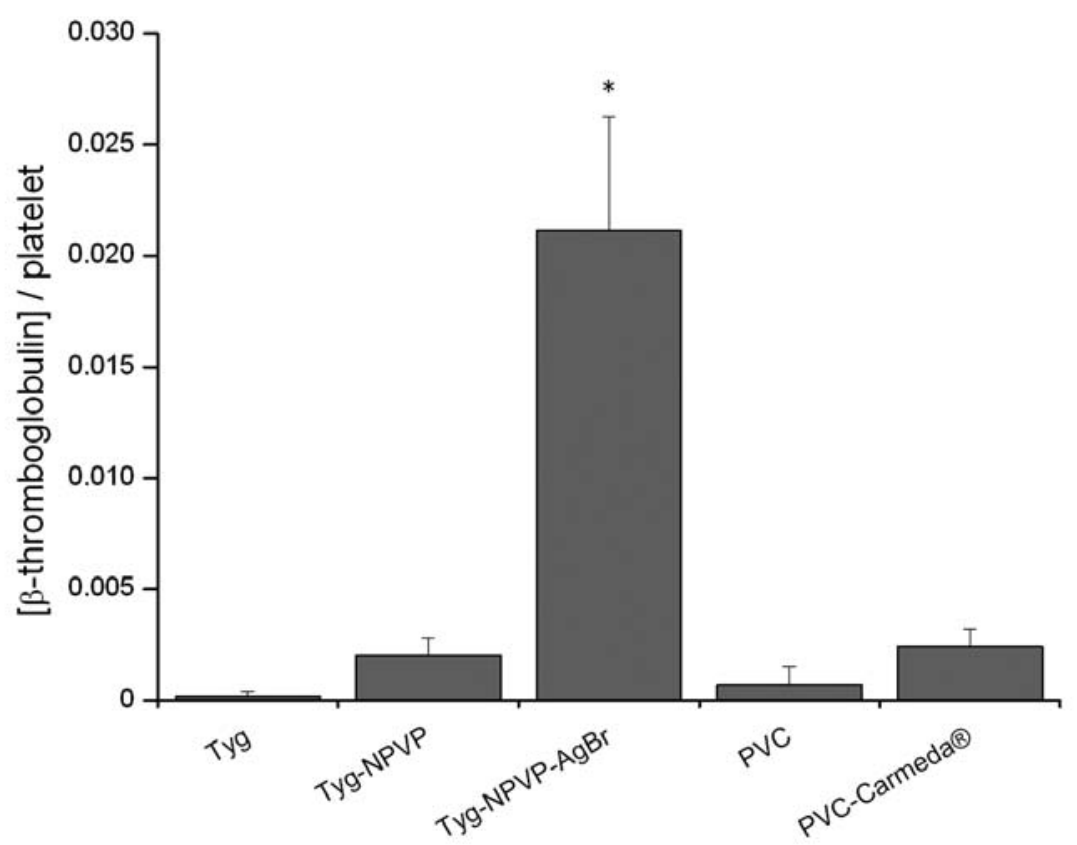

Figure 4. Analysis of platelet activation. Levels of $\beta$-thromboglobulin were elevated in Tyg-NPVP-AgBr catheters. Experiments were performed in triplo. Data from the platelet activation assay were corrected for baseline platelet activation. For each catheter group, concentrations of $\beta$-thromboglobulin $(\mathrm{IU} / \mathrm{mL}$ ) were divided by the mean concentration of platelets $/ \mathrm{cm}^{2}$ present on the respective catheter surfaces. Data are presented as mean \pm standard deviation. $\left({ }^{*}\right)$ Concentration $\beta$-thromboglobulin/platelet significantly different compared to Tyg and PVC.

On the other hand, the Tyg-NPVP-AgBr surface induced marked activation, as is evidenced by an approximately ten-fold higher amount of $\beta$-thromboglobulin released per platelet. Remarkably, the relatively high levels of $\beta$-thromboglobulin in the plasma coincide with a distorted morphology of platelets adhered at the surface. This is plausible, since platelet lysis will result in leakage of the cellular contents, including $\beta$-thromboglobulin, into the surrounding medium. 


\section{Thrombin generation}

These experiments were done with fresh PRP from 6 healthy male donors, and four different biomaterials: Tyg-NPVP-AgBr coated tubes, TygNPVP coated tubes, Tyg tubes, and the HemoGlide ${ }^{\text {TM }}$ catheter. Measurement of the thrombin generation curve is one of the best methods to assess thrombogenicity of artificial materials ${ }^{18,19}$. Here, we chose to use the static assay and PRP ${ }^{8,12}$. In principle, thrombin generation can also be measured in a flow system, using whole blood. However, due to the relatively large bore of our samples, the dynamic assay would have required more than $1 \mathrm{~L}$ of fresh human blood per sample, which was clearly impossible.

As is well-known, the contact of blood with a foreign surface triggers the intrinsic pathway ${ }^{19}$. The intrinsic and extrinsic pathways coincide at the level of factor Xa. Then, downwards in the coagulation cascade, the result is the formation of thrombin, which will ultimately catalyze the conversion of fibrinogen into fibrin.

The results of our thrombin generation measurements are shown in Figure 5. Note that these graphs plot the thrombin generation lag-times (TGTlag, vide supra) as measured at least in four-fold per donor and per material.

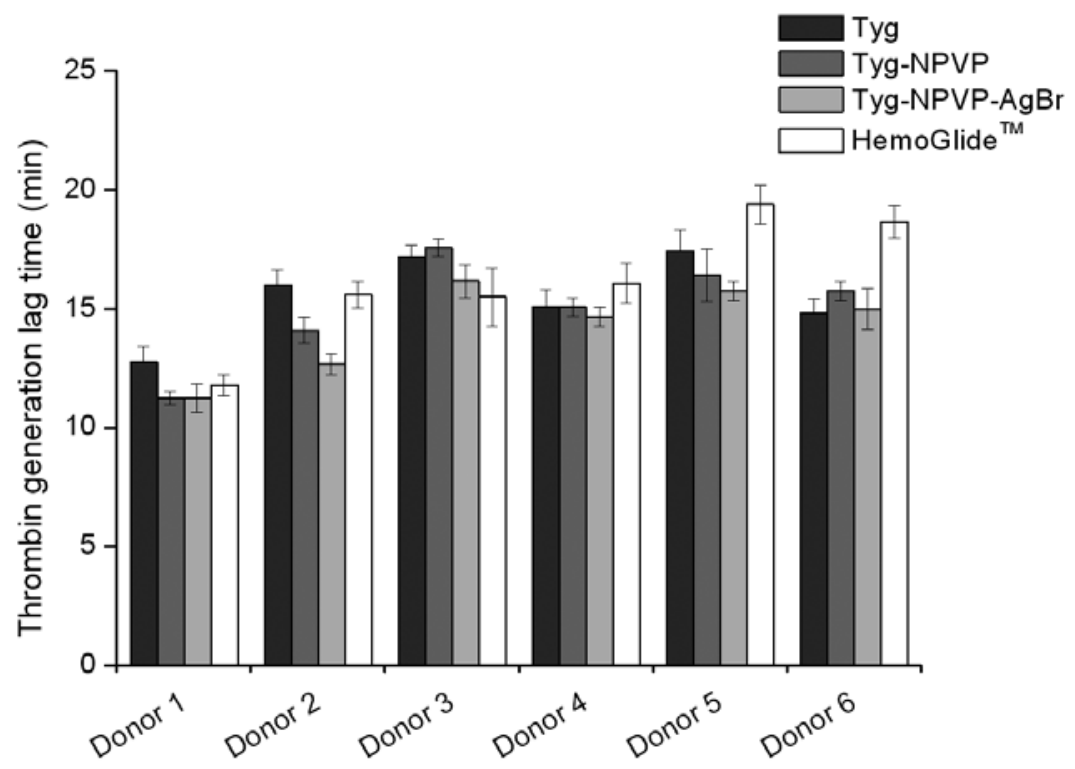

Figure 5. Thrombin generation assays for 6 different donors. Thrombin generation lag times (TGTlag) are presented for Tyg, Tyg-NPVP, Tyg-NPVP-AgBr and HemoGlide ${ }^{\text {TM }}$ catheters. Data are shown as mean \pm standard deviation. Experiments were performed in six-fold. 
The data in Figure 5 reveal that the TGTlag values show a considerable donor-dependency. For example, comparing the data that were obtained with the Tyg material (a control), it is seen that the TGTlag times rank as follows: donor $1(13+/-1 \mathrm{~min})<$ donor $4(15+/-2 \mathrm{~min})=$ donor $6(15+/-2 \mathrm{~min})<$ donor $2(16+/-2 \mathrm{~min})<\operatorname{donor} 3(17+/-2 \mathrm{~min})=$ donor $5(17+/-2 \mathrm{~min})$. In other words, fastest clotting was observed with the PRP from donor 1, while slowest clotting was observed with the PRP from donors 3 and 5. Donor variations of approximately the same magnitude have been observed previously ${ }^{8}$.

There is no consistent effect of the NPVP coating on the thrombin generation lag time. For donors 1, 2 and 5, a slight decrease of TGTlag is observed, whereas no effect was found for donors 3 and 4. For donor 6, the TGTlag was found to increase slightly upon application of the NPVP coating. Incorporation of the $\mathrm{AgBr}$ nanoparticles in the NPVP coating has a small accelerating effect on thrombin formation, at least for donors 2, 3, 5 and 6. With the PRP of donors 1 and 4, we found no effect of the TGTlag values due to the presence of the $\mathrm{AgBr}$ nanoparticles. Finally, the TGTlag values measured with the HemoGlide ${ }^{\mathrm{TM}}$ catheter material are only slightly longer than those measured for Tyg-NPVP-AgBr. Considerable differences were found for donors 2, 5 and 6.

\section{Discussion}

Our evaluation of the events that occur after contacting fresh human PRP (which contains the major players in hemostasis, i.e. the proteins that constitute the coagulation cascade, and the blood platelets) with the materials Tyg, Tyg-NPVP, Tyg-NPVP-AgBr and control materials has led to several new insights. Perhaps the most important observation was that the Tyg-NPVP-AgBr surface induces activation and disruption of the platelets. This was most evident from our measurements of $\beta$-thromboglobulin, a protein that is released by activated platelets ${ }^{20}$. Examining the surface of Tyg-NPVP-AgBr with scanning electron microscopy (after an incubation time of $1 \mathrm{~h}$ ) showed extensively distorted morphologies of the adhered platelets. It is likely that this shape coincides with a high degree of activation and disruption. Previous work of Sambhy, et al. ${ }^{1}$ describes elution of $\mathrm{Ag}^{+}$from the NPVP-AgBr coating. Whether elution of $\mathrm{Ag}^{+}$and, subsequently, platelet activation in suspension, can explain the observed platelet activating effects is an interesting point. This possibility can be ruled out on the basis of three combined arguments: (i) $\mathrm{Ag}^{+}$have antimicrobial action if the concentration exceeds $100 \mathrm{nM}^{8}$; (ii) $\mathrm{Ag}^{+}$lead to platelet activation if their concentration exceeds $100 \mu \mathrm{M}{ }^{8}$; (iii) the $\mathrm{Ag}^{+}$ concentration as a result of leaching out of NPVP-AgBr coatings is approximately $4 \mathrm{ppm}^{1}$. The concentration of $4 \mathrm{ppm}$ corresponds with $0.3 \mu \mathrm{M}$. This implies that media around these coatings are antimicrobial but not inducing platelet activation. Hence, we adhere to the explanation that the NPVP-AgBr 
surfaces have platelet activating and disrupting features, not the media surrounding these surfaces.

Furthermore, we found that the thrombin generation assay could not discriminate between the "platelet-friendly" surfaces on one hand (i.e., Tyg, TygNPVP and the other controls), and the platelet-disrupting surface Tyg-NPVP$\mathrm{AgBr}$ on the other hand. This may indicate that the NPVP-AgBr induced activation also precludes the phospholipid transition in the platelet membrane; this transition (also known as flip-flop mechanism) is known to be mandatory in order to turn the platelet's membrane into a procoagulant surface ${ }^{21,22}$. The results from this study reveal that measurement of thrombin generation curves alone is not sufficient to assess the blood compatibility of artificial surfaces. It is absolutely necessary to examine the platelets (and especially their status in terms of activation) as well.

\section{Conclusions}

The pronounced antimicrobial activity of NPVP-AgBr composites makes these materials attractive candidates as coatings for catheters. The observed activating and disrupting effect on blood platelets disqualifies these materials as coatings for central venous catheters. Probably, pyridinium polymer/AgBr composites may prove extremely useful as antibacterial surfaces in applications outside the biomedical field, in which contact with cells other than bacteria is essentially irrelevant.

\section{Acknowledgements}

The authors would like to thank Mr. Stefan G.J.A. Camps for his assistance during the laboratory experiments.

\section{Table of Contents Graphic}

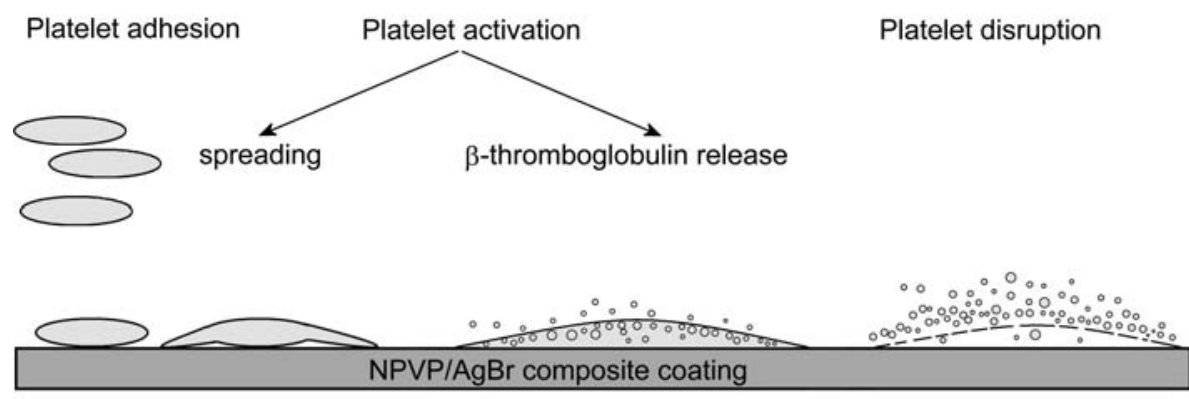




\section{References}

1 Sambhy, V., MacBride, M. M., Peterson, B. R. \& Sen, A. Silver bromide nanoparticle/polymer composites: dual action tunable antimicrobial materials. J Am Chem Soc 128, 9798-9808, (2006).

2 Acedo Sanchez, J. D., Batlle, J. F. \& Feijoo, J. B. Catheter-related thrombosis: a critical review. Support Cancer Ther 4, 145-151, (2007).

3 Burns, K. E. \& McLaren, A. A critical review of thromboembolic complications associated with central venous catheters. Can J Anaesth 55, 532-541, (2008).

4 Ruschulte, $\mathrm{H}$. et al. Prevention of central venous catheter related infections with chlorhexidine gluconate impregnated wound dressings: a randomized controlled trial. Ann Hematol 88, 267-272, (2009).

5 Zingg, W., Cartier-Fassler, V. \& Walder, B. Central venous catheter-associated infections. Best Pract Res Clin Anaesthesiol 22, 407-421 (2008).

6 Sambhy, V., Peterson, B. R. \& Sen, A. Antibacterial and hemolytic activities of pyridinium polymers as a function of the spatial relationship between the positive charge and the pendant alkyl tail. Angew Chem Int Ed Engl 47, 1250-1254, (2008).

$7 \quad<$ www.bardaccess.com>

8 Stevens, K. N. et al. The relationship between the antimicrobial effect of catheter coatings containing silver nanoparticles and the coagulation of contacting blood. Biomaterials 30, 3682-3690, (2009).

9 Alban, S. Pharmacological strategies for inhibition of thrombin activity. Curr Pharm Des 14, 1152-1175 (2008).

10 Kalb, M. L., Potura, L., Scharbert, G. \& Kozek-Langenecker, S. A. The effect of ex vivo anticoagulants on whole blood platelet aggregation. Platelets 20, 7-11, (2009).

11 Kopp, R. et al. Effect of hirudin versus heparin on hemocompatibility of blood contacting biomaterials: an in vitro study. Int J Artif Organs 28, 1272-1277 (2005).

12 Aldenhoff, Y. B., Hanssen, J. H., Knetsch, M. L. \& Koole, L. H. Thrombus formation at the surface of guide-wire models: effects of heparin-releasing or heparin-exposing surface coatings. J Vasc Interv Radiol 18, 419-425, (2007).

13 Stevens, K. N., Aldenhoff, Y. B., van der Veen, F. H., Maessen, J. G. \& Koole, L. H. Bioengineering of improved biomaterials coatings for extracorporeal circulation requires extended observation of blood-biomaterial interaction under flow. J Biomed Biotechnol 2007, 29464, (2007).

14 Goodman, S. L. Sheep, pig, and human platelet-material interactions with model cardiovascular biomaterials. J Biomed Mater Res 45, 240-250, (1999).

15 Wielders, S. J., Ungethum, L., Reutelingsperger, C. P., Bevers, E. M. \& Lindhout, T. Factor Xadriven thrombin generation in plasma: dependency on the aminophospholipid density of membranes and inhibition by phospholipid-binding proteins. Thromb Haemost 98, 1056-1062, (2007).

16 Hemker, H. C. et al. The calibrated automated thrombogram (CAT): a universal routine test for hyper- and hypocoagulability. Pathophysiol Haemost Thromb 32, 249-253, (2002).

17 Rodrigues, S. N., Goncalves, I. C., Martins, M. C., Barbosa, M. A. \& Ratner, B. D. Fibrinogen adsorption, platelet adhesion and activation on mixed hydroxyl-/methyl-terminated self-assembled monolayers. Biomaterials 27, 5357-5367, (2006).

18 Saralidze, K., van Hooy-Corstjens, C. S., Koole, L. H. \& Knetsch, M. L. New acrylic microspheres for arterial embolization: combining radiopacity for precise localization with immobilized thrombin to trigger local blood coagulation. Biomaterials 28, 2457-2464 (2007).

19 Thor, A. et al. The role of whole blood in thrombin generation in contact with various titanium surfaces. Biomaterials 28, 966-974, (2007).

20 Ohkawa, R. et al. Platelet release of beta-thromboglobulin and platelet factor 4 and serotonin in plasma samples. Clin Biochem 38, 1023-1026, (2005). 
NPVP/AgBr composite coatings: disruption and activation of contacting platelets

21 Schoenwaelder, S. M. et al. Two distinct pathways regulate platelet phosphatidylserine exposure and procoagulant function. Blood 114, 663-666, (2009).

22 Zwaal, R. F., Comfurius, P. \& Bevers, E. M. Surface exposure of phosphatidylserine in pathological cells. Cell Mol Life Sci 62, 971-988, (2005). 
Hydrophilic surface coatings with embedded biocidal silver nanoparticles and sodium heparin for central venous catheters

Stevens $\mathrm{KN}^{1}$, Croes $\mathrm{S}^{2}$, Boersma $\mathrm{RS}^{3}$, Stobberingh $\mathrm{EE}^{2}$, van der Marel $\mathrm{C}^{4}$, van der Veen $\mathrm{FH}^{1}$, Knetsch $\mathrm{ML}^{5}$, Koole $\mathrm{LH}^{5}$.

1. Department of Cardiothoracic Surgery, Maastricht University Medical Centre, Maastricht, The Netherlands.

2. Department of Medical Microbiology, Maastricht University Medical Centre, Maastricht, The Netherlands.

3. Department of Internal Medicine (Hematology), Maastricht University Medical Centre, Maastricht, The Netherlands.

4. Philips Research - MiPlaza Materials Analysis, High Tech Campus, Eindhoven, The Netherlands.

5. Centre for Biomaterials Research, University of Maastricht, Maastricht, The Netherlands

Biomaterials. 2011;32(5):1264-9. 


\section{Abstract}

Central venous catheters (CVCs) have become indispensible in the treatment of neonates and patients undergoing chemotherapy or hemodialysis. A CVC provides easy access to the patient's circulation, thus enabling facile monitoring of hemodynamic parameters, nutritional support, or administration of (cytostatic) medication. However, complications with CVCs, such as bacterial bloodstream infection or thromboembolism, are common. Bloodstream infections, predominantly caused by S. aureus, are notoriously difficult to prevent and treat. Furthermore, patients receiving infusion therapy through a CVC are at risk for deep-vein thrombosis, especially of the upper limbs. Several recent clinical trials have shown that prophylactic anticoagulation (low-molecularweight heparin or vitamin $\mathrm{K}$ antagonists) is not effective. Here, we report on the systematic development of a new bifunctional coating concept that can -uniquely- be applied to make CVC surfaces antimicrobial and antithrombogenic at the same time. The novel coating consists of a moderately hydrophilic synthetic copolymer of N-vinylpyrrolidinone (NVP) and n-butyl methacrylate (BMA), containing embedded silver nanoparticles (AgNPs) and sodium heparin. The work demonstrates that the AgNPs strongly inhibit adhesion of S. aureus (reference strain and clinical isolates). Surprisingly, heparin not only rendered our surfaces practically non-thrombogenic, but also contributed synergistically to their biocidal activity. 


\section{Introduction}

Central venous catheters (CVCs) are used ubiquitously during treatment of critically ill cancer patients. According to recent estimates, more than 5 million cancer patients in the US require central venous access each year ${ }^{1}$. A similar estimate was made for Europe. CVCs offer important advantages, such as facile sustained administration of cytostatic or pain-killing medication, infusion of stem cells, continuous measurement of hemodynamic parameters, or sustained nutritional support. However, application of CVCs is associated with a significant risk for adverse effects, particularly bloodstream infection $23,4,5$ and thromboembolism ${ }^{6,7,8,910}$. In the US, approximately 80,000 CVC-related nosocomial bloodstream infections occur annually. The associated extra cost is in the range of $\$ 300$ million to $\$ 2.3$ billion per year, and the attributable mortality is around $20 \%$. On average, survivors usually remain one extra week in the intensive care unit, or $2-3$ additional weeks in the hospital.

Hence, prevention of CVC-related complications is of paramount importance. Regarding infection, preventive strategies include the use of (i), a maximum sterile barrier during CVC insertion; (ii), innovative catheter hubs, and (iii), chlorhexidine-containing cutaneous antiseptics 23,4,5,11. Moreover, strict adherence to evidence-based protocols for hygiene and sterility proved highly successful ${ }^{11}$. Prevention of thrombotic complications is mostly attempted through administration of anticoagulants during treatment ${ }^{6,7,8,9,10}$. Despite all efforts, it is evident that there is a need for improved biomaterials for the manufacture of safer catheters. Engineering into this direction must focus of the catheter's surface, which must have broad-spectrum antimicrobial activity as well as excellent blood compatibility. We describe the systematic development of new bifunctional surface coatings that -uniquely- meet these requirements.

\section{Materials \& methods}

\section{Formulation}

Six different coating solutions were prepared as follows: (i), $600 \mathrm{~mL}$ of of $10 \%$ solution of the hydrophilic copolymer (SS) in NMP was equally divided over six 500-mL glass bottles. (ii), Sodium heparin (3 x $1.5 \mathrm{~g}$, purchased from Celsus Laboratories, Cincinatti, OH, USA) was dissolved (mechanical stirring) in formamide $(75 \mathrm{~mL})$. The solution was split into 3 equal parts, and these were mixed with 3 of the SS solutions as indicated in Table 1. (iii), AgNPs (Ag6V or Ag4E; 3.0 g, purchased from Metalor SA, Neuchatel, Switzerland; Ag6V and $\mathrm{Ag} 4 \mathrm{E}$ differed with respect to the hydrophilic surface coating used for their stabilization) were dispersed in NMP and mixed with 2 of the SS solutions as 
follows: Coating \#1 (SS): SS solution (100 mL) + NMP (50 mL). Coating \#2 (SS-Hep): SS solution $(100 \mathrm{~mL})+\mathrm{NMP}(25 \mathrm{~mL})+1.5 \mathrm{~g}$ heparin dissolved in formamide (25 mL). Coating \#3 (SS-Ag6V): SS solution $(100 \mathrm{~mL})+\mathrm{NMP}(50$ $\mathrm{mL})+$ nanosilver $(1.5 \mathrm{~g})$. Coating \#4 (SS-Ag6V-Hep): SS solution (100 mL) + NMP $(25 \mathrm{~mL})+$ nanosilver $(1.5 \mathrm{~g})+1.5 \mathrm{~g}$ heparin dissolved in formamide $(25$ $\mathrm{mL})$. Coating \#5 (SS-Ag4E): SS solution $(100 \mathrm{~mL})+\mathrm{NMP}(50 \mathrm{~mL})+$ nanosilver (1.5 g). Coating \#6 (SS-Ag4E-Hep): SS solution (100 mL) + NMP (25 mL) + nanosilver $(1.5 \mathrm{~g})+1.5 \mathrm{~g}$ heparin dissolved in formamide $(25 \mathrm{~mL})$. Coatings had a thickness of $3.0 \mu \mathrm{m}$, and differed only with respect to the embedded species. Fabrication of the specimens involved three consecutive steps: (i) formulation of coating suspensions through mixing of the NVP/BMA copolymer (designated SS) + AgNPs suspended in N-methylpyrrolidone and/or sodium heparin dissolved in formamide; (ii) application of the coating onto a long (approximately $300 \mathrm{~m}$ ) and thin $(178 \mu \mathrm{m}$ diameter) stainless steel wire in a continuous process; (iii), coiling of the coated wire around a rotating mandril of $600 \mu \mathrm{m}$ diameter ${ }^{12,13,14}$.

Table 1. Atomic concentrations (percentages) measured by XPS. All data represent averages of two independent measurements.

\begin{tabular}{|c|c|c|c|c|c|c|}
\hline Entry & Surface & C1s & N1s & O1s & Ag3d & S2p \\
\hline & & & & & & $\left(-\mathrm{SO}_{3} \mathrm{H}\right)$ \\
\hline 1 & SS & 79.7 & 4.6 & 14.4 & - & 0.21 \\
\hline 2 & SS-Hep & 78.6 & 4.3 & 14.5 & - & 0.43 \\
\hline 3 & SS-Ag6V & 80.4 & 4.2 & 12.6 & 0.22 & 0.21 \\
\hline 4 & SS-Ag6V-Hep & 79.2 & 5.0 & 14.2 & 0.11 & 0.42 \\
\hline 5 & SS-Ag4E & 79.3 & 6.6 & 13.0 & 0.15 & 0.24 \\
\hline 6 & SS-Ag4E-Hep & 78.9 & 5.3 & 14.6 & 0.19 & 0.35 \\
\hline
\end{tabular}

\section{Methods and equipment}

The platelet-stabilizing anticoagulant mixture, citrate-theophillineadenosine-dipyridamole (CTAD) was purchased from Becton-Dickinson (Alphen a/d Rijn), Netherlands). Brain Heart infusion broth and MuellerHinton agar were from Oxoid BV (Badhoevedorp, Netherlands). The lactate dehydrogenase (LDH) assay was performed using the CytoTox 96 NonRadioactive Cytotoxicity Assay from Promega Benelux BV (Leiden, Netherlands). Platelet activation was quantified with the Assachrom $\beta$-TG linked immunosorbent assay (ELISA), obtained from Roche Diagnostics Nederland BV (Almere, Netherlands). Fluorescence tracings were recorded on a SpectraMax M2 spectrofluorometer (Molecular Devices, Sunnyvale, CA, USA). Absorbances were measured on an ELx808 Absorbance Microplate Reader 
(BioTek Instruments Inc., VT, USA). Sputter coating for electron microscopy was performed with a Sputter Coater 108/SE (Cressington Scientific Instruments Ltd., Watford, UK). Scanning electron microscopy was performed with a Philips XL30 instrument (Philips, Eindhoven, the Netherlands). X-ray Photoelectron Spectroscopy measurements were carried out in a Quantera SXM instrument from Ulvac-PHI (Q2). During the measurements, the angle between the axis of the analyzer and the sample surface was $45^{\circ}$. The information depth is then appr. $6 \mathrm{~nm}$. The measurements have been performed using monochromatic AlK $\alpha$ radiation in High Power mode (100 Watt, measuring spot $100 \mu \mathrm{m}$, scanned over $1400 \mu \mathrm{m} \times 500 \mu \mathrm{m})$.

\section{Experiments with bacteria}

Overnight cultures were prepared by inoculation of a bacterial colony into $10 \mathrm{~mL}$ growth medium ( $37 \mathrm{~g} / \mathrm{L}$ heart infusion broth). From this culture, a bacterial suspension of $10^{7} \mathrm{CFU} / \mathrm{mL}$ was prepared in $0.9 \% \mathrm{NaCl}$. Coated coils (length $30 \mathrm{~mm}$ ) were incubated at $37^{\circ} \mathrm{C}$ for $60 \mathrm{~min}$ in $5 \mathrm{~mL}$ bacterial suspension, in a shaking incubator. The samples were subsequently transferred to a fresh volume of $5 \mathrm{~mL} 0.9 \% \mathrm{NaCl}$, and vortexed for $1 \mathrm{~s}$. Vortexing resulted in detachment of loosely adhered bacteria. Then, the samples were transferred again to fresh volumes of $5 \mathrm{~mL}$ of $0.9 \% \mathrm{NaCl}$, and left for $4 \mathrm{~h}$ under continuous shaking. Subsequently, the catheter samples were carefully removed, washed in $0.9 \% \mathrm{NaCl}$, and rolled over a Mueller-Hinton blood agar plate $(38 \mathrm{~g} / \mathrm{L}$ Mueller-Hinton agar, $5 \%$ defibrinated sheep blood). Plates were incubated overnight and photographed and evaluated on the next day. The six sample groups were subjected to these tests in three-fold.

\section{Thrombin generation experiments}

Freshly prepared human platelet-rich blood plasma (PRP) was used in the thrombin generation assays. There were two donor groups: a first group consisting of 5 healthy male volunteers (ages 22, 23, 24, 25, and 51 years), who were non-smokers and non-users of any drugs that could possibly influence hemostasis. The second group consisted of a cohort of 9 patients who were under treatment in the Maastricht University Medical Centre+. These patients all received high-dose cytostatic medication through a central venous catheter; patient data are summarized in Table 2. Informed consent was obtained in accordance with the Declaration of Helsinki, and the study was approved by the Maastricht University Medical Centre+ ethical committee. The volunteers each donated appr. $40 \mathrm{~mL}$ blood through venipuncture. The collection tubes contained citrate for anticoagulation (end-concentration $0.013 \mathrm{M}$ citrate). The patients donated $20 \mathrm{~mL}$ blood each. In these cases, Vacutainers containing 
citrate for anticoagulation were used. PRP was isolated through centrifugation (200g, $15 \mathrm{~min}$, room temperature). PRP was carefully transferred into new tubes and kept at $37^{\circ} \mathrm{C}$ until further use. Thrombin generation experiments were done according to a one-donor-on-one-day scheme. On such an experimental day, 30 thrombin generation curves were measured ( 6 experimental coatings, each experiment in five-fold). The experiments were done in 4 consecutive steps: (i), Five pieces of $25 \mathrm{~mm}$ were cut out of each of the six different coils. Then, each piece was cut further into five approximately equal pieces, and these were transferred into one single well of a 96 well plate, (ii), The fluorogenic substrate for thrombin, Z-Gly-Gly-Arg-AMC (a product of Bachem Holding AG (Bubendorf, Switzerland; ref. I-1140) was added to the citrated PRP to a final concentration of $400 \mu \mathrm{M}$. Then, the PRP was "recalcified" through adding $\mathrm{CaCl} 2$ stock solution $(0.5 \mathrm{M})$ up to a final concentration of $20 \mathrm{mM} \mathrm{Ca}^{2+}$. Consequently the mechanism for intrinsic (i.e. biomaterial-surface-induced) coagulation is no longer inhibited, (iii), The PRP was then rapidly distributed over the wells; $200 \mu \mathrm{L}$ was transferred into each well. All pieces of the coiled wire were submersed in PRP. Wells without coil-samples served as controls. (iv), Fluorescence tracings were recorded at $37^{\circ} \mathrm{C}$. Wavelengths of excitation and measuring were 368 and $460 \mathrm{~nm}$, respectively. Data were collected every $30 \mathrm{~s}$; the plate was gently shaken for $2 \mathrm{~s}$ prior to each measurement. The fluorescence intensity was converted into nanomolar concentrations of thrombin. This technique resulted in a thrombin generation curve for each well.

\section{Statistics}

Statistical analysis was performed using Kruskal-Wallis analysis with Dunn's post test for between group comparisons. Separate analyses were performed for thrombin generation experiments performed with PRP from different donors. A p-value less than 0.05 (two-tailed) was considered significant. 
Table 2. Data on patients/blood donors.

\begin{tabular}{|c|c|c|c|c|c|}
\hline Patient & $\begin{array}{l}\text { Age } \\
\text { (gender) }\end{array}$ & $\begin{array}{l}\text { Length } \\
\text { (m)/mass (kg) }\end{array}$ & Specified malignancy & $\begin{array}{l}\text { Thrombocyte } \\
\text { counts }\left(10^{9} / \mathrm{L}\right)^{b}\end{array}$ & $\begin{array}{l}\text { Leukocyte counts } \\
\left(10^{9} / \mathrm{L}\right)^{\mathrm{c}}\end{array}$ \\
\hline 1 & $50(f)$ & $1.64 / 47$ & $\mathrm{AML}^{\mathrm{a}}$ & 134 & 1.4 \\
\hline 2 & $61(\mathrm{~m})$ & $1.72 / 64$ & Histiocytic sarcoma & 52 & 0.2 \\
\hline 3 & $61(f)$ & $1.58 / 53$ & AML & 145 & 3.0 \\
\hline 4 & $60(\mathrm{~m})$ & $1.85 / 104$ & AML & 51 & 1.0 \\
\hline 5 & $58(f)$ & $1.63 / 69$ & Amyloidosis & 107 & 10.5 \\
\hline 6 & $66(\mathrm{~m})$ & $1.83 / 65$ & $\begin{array}{l}\text { MGUS + C1-esterase } \\
\text { deficiency }\end{array}$ & 281 & 4.5 \\
\hline 7 & $76(\mathrm{~m})$ & $1.75 / 60$ & $\begin{array}{l}\text { Mantle cell non- } \\
\text { Hodgkin's lymphoma }\end{array}$ & 120 & 0.8 \\
\hline 8 & $44(\mathrm{~m})$ & $1.80 / 77$ & AML & 252 & 6.2 \\
\hline 9 & $55(\mathrm{~m})$ & $1.72 / 109$ & Multiple myeloma & 27 & 1.5 \\
\hline
\end{tabular}

Patient \#5 was the only patient receiving anticoagulant medication (low-molecular weight heparin) at the time of blood sampling.

a $\mathrm{AML}=$ acute myeloid leukemia.

b Normal thrombocyte concentration: $150-450 \times 10^{9} / \mathrm{L}$.

c Normal leukocyte concentration: 4.5-10 x 10\% $/ \mathrm{L}$.

\section{Results}

The continuous coating process afforded a huge inventory of virtually identical specimens for every formulation. This approach distinguishes this study from other investigations on biomaterial surface coatings obtained through dipping or spraying, which are discontinuous methods that generally introduce substantial noise in subsequent experimental data. Specimens were first studied with X-ray photoelectron spectroscopy (XPS). Fig. 1a shows a representative wide-scan spectrum. Data were collected with a spot size of only $100 \mu \mathrm{m}$ at two positions per specimen, i.e., on the top of two individual windings. The information depth was approximately $6 \mathrm{~nm}$. Fig. $1 \mathrm{~b}$ shows narrow-scan XPS spectra of SS-Ag4E-Hep (sulfur and silver lines). The apparent atomic concentrations are compiled in Table 1. All duplicate measurements were in good agreement. As expected, only specimens 3 - 6 expose silver at their surface. The specimens containing heparin (2, 4 and 6$)$ show clearly elevated concentrations of sulfur $\left(-\mathrm{SO}_{3} \mathrm{H} ; 167.9 \pm 0.1 \mathrm{eV}\right)$ at their surfaces $(0.43,0.42$ and $0.35 \%$ respectively, vs. $0.21,0.21$ and $0.24 \%$ for specimens 1, 3, and 5). Hence, XPS confirms the presence of heparin at the surface of 2, 4 and 6 . To the best of our knowledge, this is the first example of the use of XPS to verify the presence of heparin in the outermost regions of biomaterial surface coatings. The presence of sulfur traces in specimens 1, 3, and 5 can be attributed to the binding layer of poly(ethersulfone), which is routinely used in the continuous coating procedure. 
a

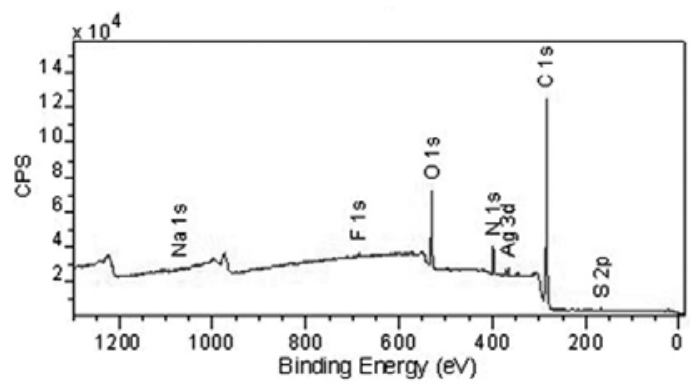

b
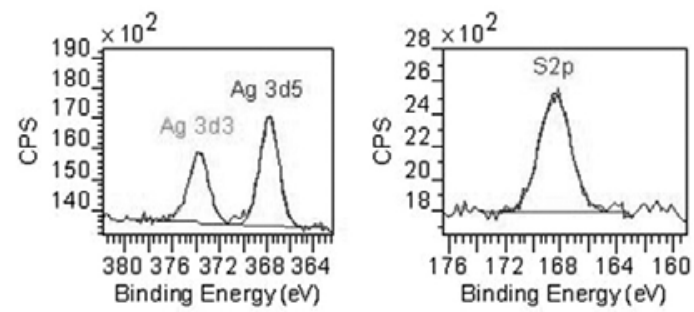

Figure 1. (a) Wide scan XPS spectrum of the typical specimen SS-Hep-Ag4E. A small spot size $(100 \mu \mathrm{m})$ was used, which enabled scanning on the top of one of the specimen's windings. (b) Narrow scan X-ray photoelectron spectra of the outermost layer of the SS-Hep-Ag4E surface. Left: Ag3d doublet due to surfaceexposed AgNPs. Right: S2p peak due to the $\mathrm{SO}_{3} \mathrm{H}$ groups of heparin. 


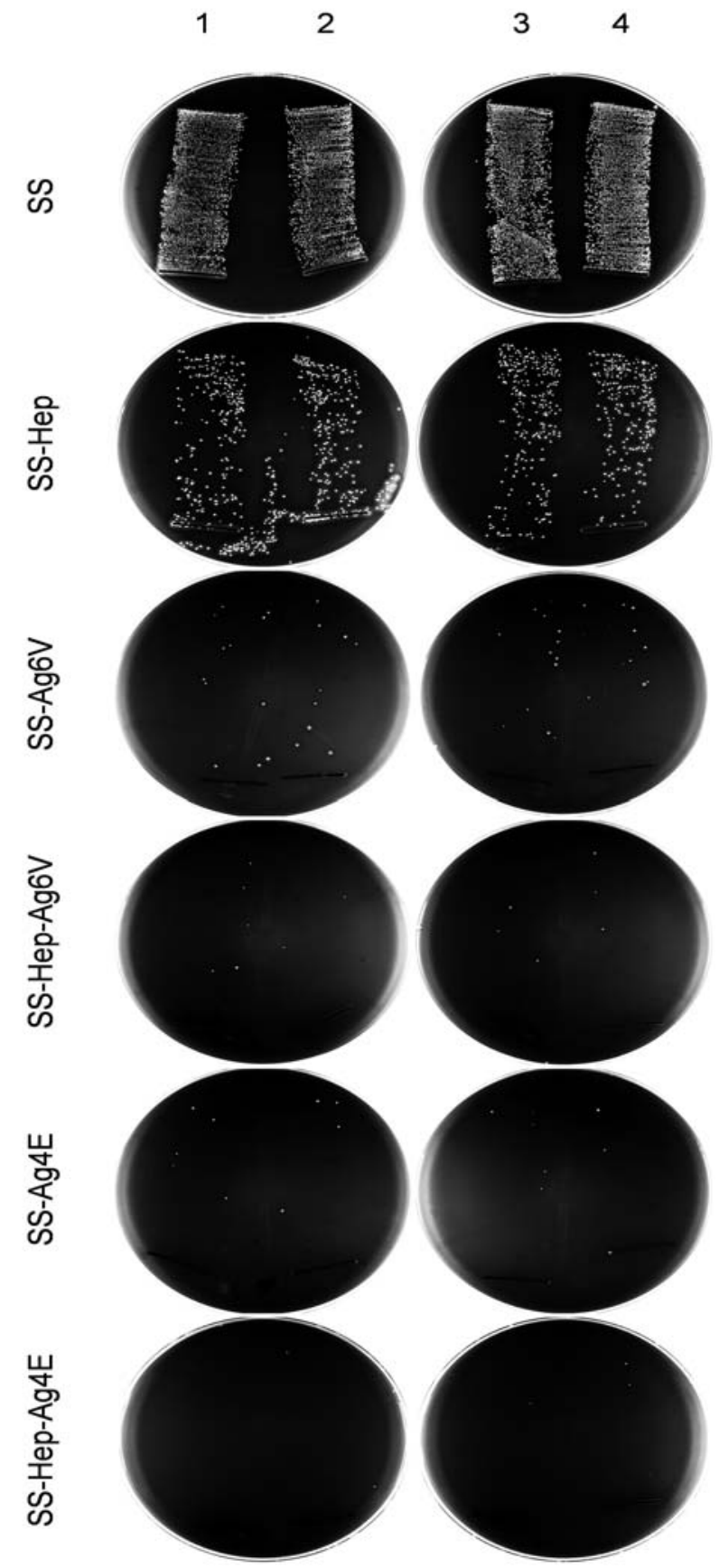

Figure 2. Bacterial colonies that developed on the agar plates (roll-plate method according to Ref. ${ }^{15}$ ). Experiments were done with 7 different coatings (one per row), and in four-fold (four columns). The specimens were carefully rolled from the upper side to the lower side of the dishes, thereby releasing bacteria that generated visible colonies afterwards. Note the presence of many colonies for the SS coating, compared to the SS-Hep coating. Bactericidal effects of the AgNPs are clearly visible. 
The roll-plate method according to Maki et al. ${ }^{15}$ was used to assess adherence of the reference strain S. aureus ATCC 29213 to the different coatings. This technique visualizes bacterial colonies on each roll track, provided that bacterial adhesion occurred during the incubation phase. Our cylindrical specimens were convenient to use in this assay, and data-reproducibility was excellent. Virtually no bacteria could be found on the agar plates for SS-Ag6V, SS-Ag6V-Hep, SS-Ag4E, and SS-Ag4E-Hep, whereas bacteria were abundantly present on the agar plates for SS-Hep and especially on SS (Fig. 2). These data confirm the strong antibacterial activity of the AgNPs Ag6V and Ag4E. Furthermore, it appeared that heparin exerts a pronounced antibacterial effect by itself (compare SS and SS-Hep). The antimicrobial effects of AgNPs and heparin are additive: it is clear that much less bacteria adhered to the SS-Ag6VHep surface than to SS-Ag6V alone. Analogously, SS-Ag4E-Hep has less adhered bacteria (if any are present at all) than SS-Ag4E. At first sight, we were puzzled by this heparin effect; several recent papers pointed out that heparin per se has no antibacterial effects ${ }^{16}$, and even that heparin may promote biofilm formation, especially for S. aureus ${ }^{17,18}$. Interestingly, recent work of Schmidtchen et al. may explain our observations in terms of an indirect effect of heparin: proteins carrying structural characteristics that impart affinity for heparin (positive charges and consensus regions such a the Cardin motif or the Weintraub motif), also have antimicrobial properties ${ }^{19,20}$. For example, heparinbinding plasma proteins such as fibronectin, vitronectin, protein-C inhibitor and von Willebrand factor are biocidal for Gram-positive and Gram-negative bacteria. Thus, if incubation of our coatings in plasma first leads to adsorption of proteins with an affinity for heparin, then these proteins introduce biocidal

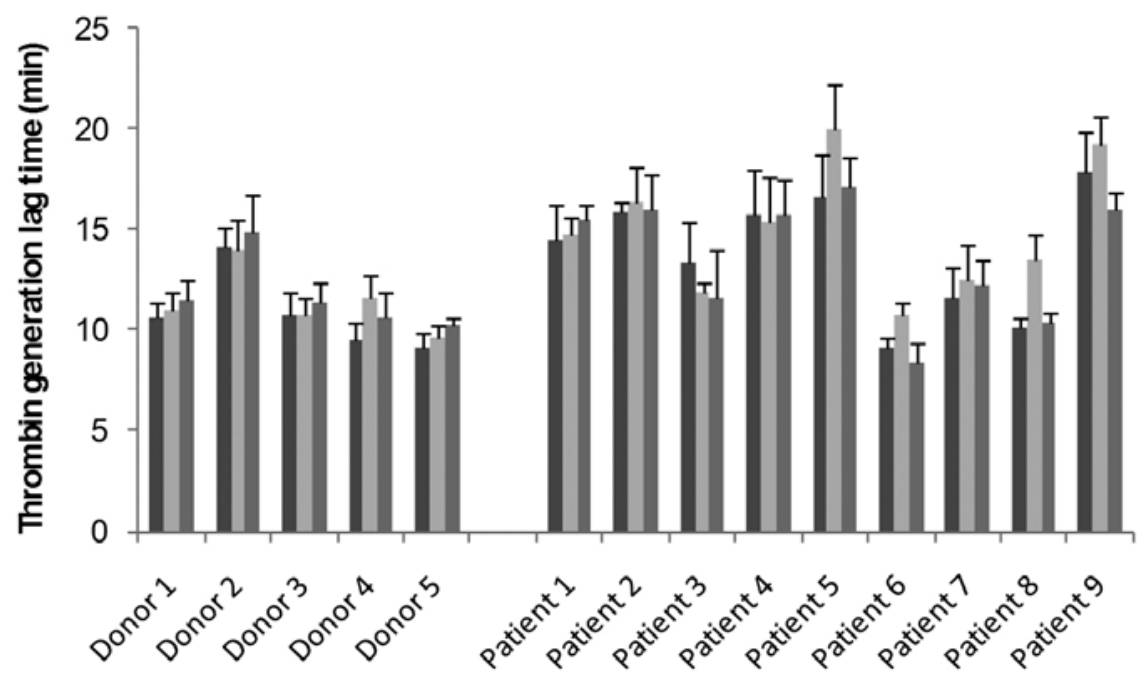

Figure 3. Thrombin generation in PRP from 5 healthy volunteers, and 9 patients on high-dose chemotherapy against a hematological malignancy. Thrombin generation lag times measured for coatings SS (left bars), SS-Ag6V (middle bars), and SS-Ag4E (right bars). 
activity at the coating's surface in turn. Next to S. aureus ATCC 29213, we studied two clinical isolates (MRSA BF110 and MSSA BF45), i.e., S. aureus bacteria that were isolated from patients with a catheter-associated bloodstream infection. The biocidal effects of our materials on the clinical isolates were, in fact, stronger than those found for the reference strain.

The six different surfaces were subjected to a thrombin generation assay 12,14. We used platelet-rich blood plasma (PRP) from 5 healthy volunteer donors and from 9 patients undergoing high-dose chemotherapy against a hematological malignancy in the Maastricht University Medical Centre+ (Table 2). We measured so-called thrombin generation curves ([thrombin] vs. time) in six-fold for every specimen-PRP combination. Thrombin lag times, i.e. time elapsing between the moment of recalcification and the moment at which [thrombin] becomes $>2 \mathrm{nM}$, were abstracted from all curves ${ }^{12,14}$. Note that the assay measures intrinsic activation of the coagulation system: the biomateral's surface is the only trigger for thrombin formation (i.e., the assay uses no tissue factor or any other procoagulant agent). As we advocated previously, the thrombin lag time is a valuable measure of the thrombogenicity of the biomaterial under investigation ${ }^{12,14}$. The longer the lag time, the higher the level of blood compatibility in vitro. Fig. 3 compiles lag times measured for our heparin-free specimens in contact with PRP from volunteer donors and patients. The volunteer data show a clear donor-dependency, which is in agreement with previous findings ${ }^{14}$. All heparin-containing specimens, for volunteer donor PRP and patient-derived PRP, had lag times $>60 \mathrm{~min}$, revealing that the anticoagulant activity of heparin is by no means neutralized or significantly compromised by the AgNPs. The thrombin lag times measure for the patients also show considerable spreading. Data measured for patients 3, 6, 7 and 8 compare well with those of the volunteers, others were somewhat longer with appr. 20 min max. Taken together, all thrombin generation lag times are grossly similar despite the fact that most patients show clear leukopenia and/or thrombocytopenia (Table 2). This reflects, albeit from an unusual point of view, that the regulation mechanism of the blood coagulation system manages to maintain hemostatic balance, even under conditions of severe illness and high-dose chemotherapy. Closer inspection of the patient data showed that one of the patients (\#5) received low-molecular-weight heparin at the time of blood sampling. For the other 8 patients, the thrombin lag times appeared to correlate inversely with the thrombocyte counts $\left(\mathrm{r}^{2}=0.76\right.$; Fig. 4). A similar but weaker correlation $\left(r^{2}=0.45\right)$ was found between the thrombin lag times and the leukocyte counts of these patients. This may point out that patients with normal or close-tonormal platelet (and leukocyte) concentrations are likely to be at the largest risk for catheter-associated thrombus formation. Clinical testing of this hypothesis falls outside the scope of this study but is certainly worthwhile: if true, then simple thrombocyte counting would provide an indication whether or not to provide prophylactic anticoagulation during the catheter-based treatment period (e.g. through infusion of low-molecular-weight heparin). 


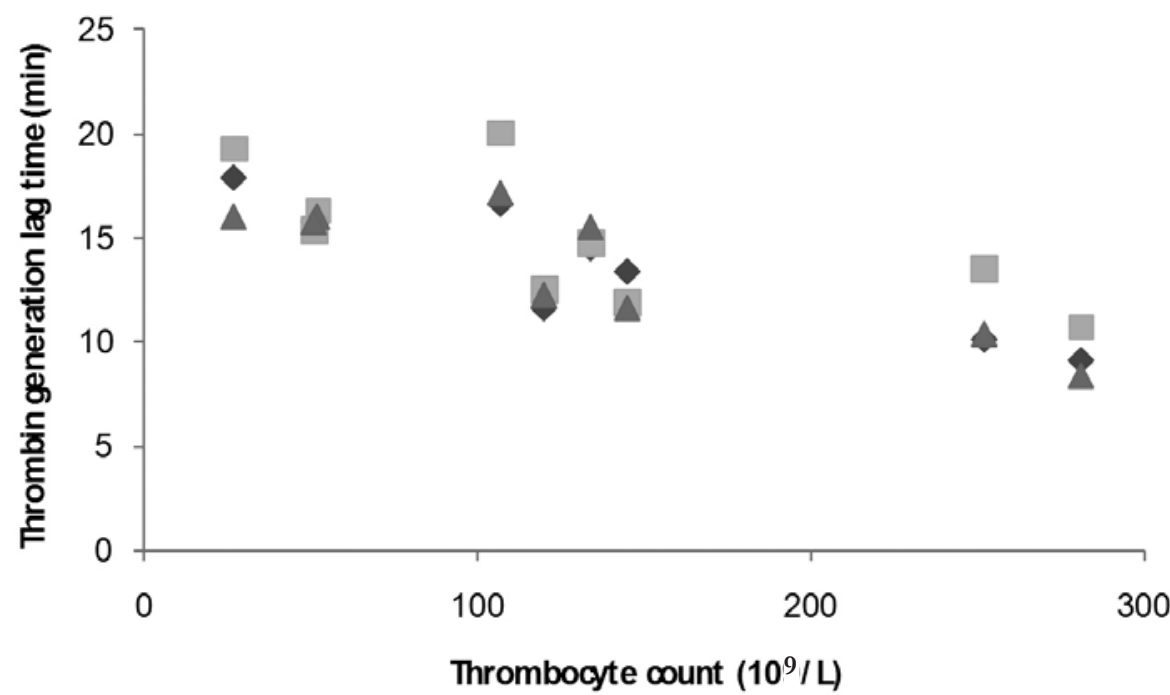

Figure 4. Averaged thrombin lag times for the 9 patients (viz. Fig. 3) plotted vs. thrombocyte counts. Thrombin lag times were measured for SS (dots), SS-Ag6V (squares), and SS-Ag4E (triangles).

Adhesion of blood platelets to the 6 experimental coatings was studied further with PRP from healthy donors \#2 and \#3. The SS surface had the largest density of adherent platelets (LDH assay, appr. 20,000/cm coil). Inclusion of heparin (SS-Hep) or AgNPs (SS-Ag6V) reduced this number to appr. 6,000/cm coil and 5,000/cm coil, respectively. The combination of heparin and AgNPs (Ag6V) reduced the density of adhered platelets further (down to appr. 2,000/ cm coil). Remarkably, the use of Ag4E nanoparticles per se (SS-Ag4E) already reduced the density of adhered platelets to appr. 2,000/cm coil. No further effect due to inclusion of heparin (SS-Hep-Ag4E) could be observed.

Scanning electron microscopy showed that platelets on the SS surface were mostly discrete with partly spread morphology. Small pseudopodia by which they attach to the surface were observed. It was very difficult to discern platelets on the three heparin-containing surfaces. On the other hand, adherent platelets could be found easily on the two coatings that contain merely AgNPs (SS-Ag6V and SS-Ag4E). These platelets were mostly associated with distorted morphologies as was observed previously ${ }^{14,21,22}$.

\section{Discussion}

Hydrophilic surface coatings for medical devices have drawn widespread attention during the last years. Adherent hydrophilic coatings provide catheters and guidewires with a "slippery-when-wet' lubricious surface, and this is extremely important for indwelling catheters, but also for delicate interventional 
procedures, such as percutaneous transluminal coronary angioplasty (PTCA) and super-precise embolization of solid tumors. New developments are focused on embedding of active components in hydrophilic surface coatings. This principle can lead to controlled release of active agents from the device's surface, and/or to exposure of active molecules at the device's surface. The combination of hydrophilic coatings with heparin can lead to anticoagulant and lubricious surfaces.

Research on drug-containing hydrophilic surface coatings requires a highly accurate and reproducible coating protocol. Obviously, variations of the coating quality (thickness, adherence, etc.) would generate substantial experimental noise, which could easily obscure effects of active constituents of the coating. Our extrusion-like coating procedure to apply NVP/BMA copolymers as uniform thin layers on thin metallic wires generates a platform of precisely defined experimental coatings. These can be used in detailed comparative studies. For instance, the hydrophilic nature of the materials can be tuned through the composition: the molar ratio NVP (hydrophilic) : BMA (hydrophobic) determines parameters like contact angle, aqueous swelling ratio, etc. NVP/BMA coatings can be engineered such that the bio-active compound is either slowly released upon immersion in an aqueous medium, or remains immobilized at the coating's surface. This principle has already led to the development of, e.g., new devices for controlled delivery of drugs to the tear film of the eye, and heparin releasing/exposing guidewires for intravascular use featuring an excellent level of blood-compatibility ${ }^{12,23,24}$.

Here, we studied NVP/BMA coating formulations in which silver nanoparticles and sodium heparin were co-embedded, with the aim to develop coatings for medical devices that effectively combine antimicrobial AND antithrombogenic features. Our data reveal that AgNPS and heparin can be combined effectively. Interestingly, synergetic effects of heparin and nanosilver were observed: (i), heparin enhances the biocidal activity of the AgNPs, and (ii), no lysis of contacting thrombocytes occurs.; rupture of platelets was observed previously with NVP/BMA coatings that merely contain embedded AgNPs. In addition, it is clear that our methodology can readily be scaled-up and transformed into an industrial procedure. We anticipate that this work can provide the basis for the development of safer central venous catheters. There is a clear unmet need for biomaterials and surfaces that combine excellent biocidal properties with zero or close-to-zero thrombogenicity in vivo. Improved CVCs will, next to other measures to prevent infection and thromboembolism, help to improve treatment of many critically ill patients, and to reduce health care cost, especially when prolonged hospitalization can be prevented indeed. 


\section{Conclusion}

Hydrophilic surface coatings for medical devices featuring both biocidal and non-thrombogenic behavior can be prepared. The methodology as described in this work can be scaled-up and transformed into an industrial procedure. This work may help to realize the goal of manufacturing catheters and other medical devices that combine excellent biocidal properties with zero or close-to-zero thrombogenicity in vivo.

\section{Acknowledgements}

Part of this work was financed by the Deutsche Forschungsgemeinschaft through the Graduiertenkolleg "BioInterfaces - Detektion und Steuerung Grenzflächeninduzierter, Biomolekularer und Zellulärer Funktionen" (GRK 1035/2). The Universities of Aachen, Liège and Maastricht cooperate within this Graduiertenkolleg. 


\section{References}

1 Frasca, D., Dahyot-Fizelier, C. \& Mimoz, O. Prevention of central venous catheter-related infection in the intensive care unit. Crit Care 14, 212, (2010).

2 Casey, A. L., Mermel, L. A., Nightingale, P. \& Elliott, T. S. Antimicrobial central venous catheters in adults: a systematic review and meta-analysis. Lancet Infect Dis 8, 763-776, (2008).

3 Geffers, C. et al. Use of central venous catheter and peripheral venous catheter as risk factors for nosocomial bloodstream infection in very-low-birth-weight infants. Infect Control Hosp Epidemiol 31, 395-401, (2010).

4 McGee, D. C. \& Gould, M. K. Preventing complications of central venous catheterization. N Engl J Med 348, 1123-1133, (2003).

5 Sabatier, C., Ferrer, R. \& Valles, J. Treatment strategies for central venous catheter infections. Expert Opin Pharmacother 10, 2231-2243, (2009).

6 Khorana, A. A. Cancer and thrombosis: implications of published guidelines for clinical practice. Ann Oncol 20, 1619-1630, (2009).

7 Schroeder, A. R. et al. A continuous heparin infusion does not prevent catheter-related thrombosis in infants after cardiac surgery. Pediatr Crit Care Med 11, 489-495, (2010).

8 Shivakumar, S. P., Anderson, D. R. \& Couban, S. Catheter-associated thrombosis in patients with malignancy. J Clin Oncol 27, 4858-4864, (2009).

9 Verso, M. \& Agnelli, G. Venous thromboembolism associated with long-term use of central venous catheters in cancer patients. J Clin Oncol 21, 3665-3675, (2003).

10 Yukisawa, S. et al. Upper-extremity deep vein thrombosis related to central venous port systems implanted in cancer patients. Br J Radiol 83, 850-853, (2010).

11 Mermel, L. A. et al. Clinical practice guidelines for the diagnosis and management of intravascular catheter-related infection: 2009 Update by the Infectious Diseases Society of America. Clin Infect Dis 49, 1-45, (2009).

12 Aldenhoff, Y. B., Hanssen, J. H., Knetsch, M. L. \& Koole, L. H. Thrombus formation at the surface of guide-wire models: effects of heparin-releasing or heparin-exposing surface coatings. J Vasc Interv Radiol 18, 419-425, (2007).

13 Aldenhoff, Y. B. et al. Coils and tubes releasing heparin. Studies on a new vascular graft prototype. Biomaterials 25, 3125-3133, (2004).

14 Stevens, K. N. et al. The relationship between the antimicrobial effect of catheter coatings containing silver nanoparticles and the coagulation of contacting blood. Biomaterials 30, 3682-3690, (2009).

15 Maki, D. G., Weise, C. E. \& Sarafin, H. W. A semiquantitative culture method for identifying intravenous-catheter-related infection. N Engl J Med 296, 1305-1309, (1977).

16 Sauer, K., Steczko, J. \& Ash, S. R. Effect of a solution containing citrate/Methylene Blue/parabens on Staphylococcus aureus bacteria and biofilm, and comparison with various heparin solutions. J Antimicrob Chemother 63, 937-945, (2009).

17 Diskin, C. J., Stokes, T. J., Dansby, L. M., Radcliff, L. \& Carter, T. B. Heparin and biofilm: is this the risk factor for catheter-related sepsis? Am J Kidney Dis 52, 197-198; author reply 198, (2008).

18 Shanks, R. M. et al. Heparin stimulates Staphylococcus aureus biofilm formation. Infect Immun 73, 4596-4606, (2005).

19 Andersson, E. et al. Antimicrobial activities of heparin-binding peptides. Eur J Biochem 271, 1219-1226, (2004).

20 Malmsten, M., Davoudi, M. \& Schmidtchen, A. Bacterial killing by heparin-binding peptides from PRELP and thrombospondin. Matrix Biol 25, 294-300, (2006).

21 Sambhy, V., MacBride, M. M., Peterson, B. R. \& Sen, A. Silver bromide nanoparticle/polymer composites: dual action tunable antimicrobial materials. J Am Chem Soc 128, 9798-9808, (2006). 
Chapter 5

22 Stevens, K. N., Knetsch, M. L., Sen, A., Sambhy, V. \& Koole, L. H. Disruption and activation of blood platelets in contact with an antimicrobial composite coating consisting of a pyridinium polymer and $\mathrm{AgBr}$ nanoparticles. ACS Appl Mater Interfaces 1, 2049-2054, (2009).

23 Agarwal, A. et al. Surfaces modified with nanometer-thick silver-impregnated polymeric films that kill bacteria but support growth of mammalian cells. Biomaterials 31, 680-690, (2010).

24 Pijls, R. T., Cruysberg, L. P., Nuijts, R. M., Dias, A. A. \& Koole, L. H. Capacity and tolerance of a new device for ocular drug delivery. Int J Pharm 341, 152-161, (2007). 
Hydrophilic coatings with nanosilver and heparin for central venous catheters 
General discussion 
Chapter 6

$\underset{\ddots}{=}$ 
The central theme of this thesis is the blood compatibility of surface coatings for cardiopulmonary bypass (CPB) and central venous catheters (CVCs). The use of these medical support devices is typically associated with intense blood-material contact in terms of a long duration (hours to weeks/months), and over a large surface area $\left(1.35-2.5 \mathrm{~m}^{2}\right)^{1}$. The rationale for the work presented here originated from the material-related complications of thrombosis and infection. The infectious complications do not only relate to CVCs, but also to $\mathrm{CPB}$, since the cannula's, used to channel the patient's bloodstream into the heart-lung machine, are in essence a type of central catheters. Since blood compatibility plays a role in both material-induced thrombosis and infection ${ }^{2,3,4,5}$, it is a prerequisite for patient safety and proper device functioning in both the field of $\mathrm{CPB}$ and (central venous) catheterization.

Surface coatings are a well-explored strategy to reduce thrombotic and infectious complications in the fields of $\mathrm{CPB}{ }^{6,7,8,9}$ and central venous catheterization 10,11,12,13. They can render an artificial surface more blood compatible or antimicrobial through incorporation of, respectively, antithrombotic or antimicrobial compounds 6,7,8,9,10,11,12,13. After decades of intensive research and development, various antithrombotic and antimicrobial coatings are now clinically available to lower the risk of material-induced thrombosis or infection, respectively $6,7,8,9,10,11,12,13$. None of these products, however, completely prevents thrombosis or infection ${ }^{14,15,16,17,18,19,20}$. In the daily clinical practice, the abovementioned complications continue to cause a significant annual burden in terms of morbidity, mortality, economic and healthcare costs $11,21,22,23,24,25,26,27,28,29,30$. Research, therefore, continues in both domains. Two trends were noticed at the beginning of this project.

(i) On one hand, it seems that the development of improved blood compatible coatings is stagnating. It appears difficult to develop coatings that are more blood compatible and can replace the currently available end-point attached heparin coatings, which remain the golden standard in clinical practice for almost twenty years now ${ }^{9}$. This observation lays at the basis of the work presented in Chapter 2. To be able to develop coatings with improved blood compatibility over existing products, a test model is needed to discriminate between the performances of coatings with already fair hemocompatibility.

(ii) On the other hand, the development of antimicrobial products has taken an important step forward through advancements in nanotechnology. During recent years, silver nanoparticles (AgNPs) came in the picture as a potential alternative for antibiotics, chlorhexidine or silver sulfadiazine, in antimicrobial catheter coatings $31,32,33,34,35,36,37,38$. AgNPs are believed to exert the well-known antimicrobial effects that are seen in other forms of silver (i.e. silver ions $\left.\left(\mathrm{Ag}^{+}\right)\right)$, and, in addition show exclusive physicochemical properties due to their nanodimension ${ }^{34,39}$. It is expected that AgNPs can serve as a source for 
long-term release of antimicrobial $\mathrm{Ag}^{+31,37}$. Progressive release of antibiotics and exhaust of the coating reservoir could lead to a fall in antibiotics concentration to such levels that development of bacterial resistance is likely.

Although nanosilver seems an attractive candidate for use in antimicrobial catheter coatings, literature regarding blood compatibility of such coatings was non-existent at the start of this project. In this thesis, we presented the first work evaluating the blood compatibility of coatings containing AgNPs (Chapter 3 and 4).

Prevention of catheter-related bloodstream infections (CRBSIs) through use of coatings has long been addressed solely with anti-infective or antibiotic agents $6,7,8,1,1,11,12,13$. However, since infectious and thrombotic catheter complications are interrelated, we hypothesized that a dual action coating, addressing both infection and thrombosis, could be more effective in preventing CRBSIs and thrombosis. A concept of such a dual action coating is presented in Chapter 5.

\section{Lessons learned from extended evaluation of blood compatible coatings}

Given the long blood-exposure times associated with clinical use of $\mathrm{CPB} /$ extracorporeal life support (ELS) or (central venous) catheters, the antithrombotic properties of surface coatings must remain effective for extended periods of time to avoid thrombotic - and infective - complications. When focusing purely on the hemocompatibility of a blood-contacting surface, leaving behind any need for antimicrobial function, most progress has been booked in the fields of CPB and ELS. Here the intensity of blood-material contact can take extreme proportions. The development of so-called hemocompatible coatings has contributed greatly in lowering the risk of thrombotic complications and preserving material functionality during such procedures ${ }^{15,16,17,40,41,42,43}$. Of the coating modalities available for CPB and ELS, those that involve heparin immobilisation at the material's surface seem to achieve the best results ${ }^{15,16,40,43}$. In particular the PVC-Carmeda ${ }^{\circledR}$, a coating that immobilizes heparin onto a surface with preservation of its structure, has been studied thoroughly and today has a long-standing efficacy in preventing materialinduced thrombus formation. It is generally perceived as the golden standard among the hemocompatible coatings. Albeit significant progress, however, the available hemocompatible coatings do not guarantee CPB and ELS procedures without thrombotic complications $6,7,8,9,44,45$. The use of aggressive anticoagulant therapy remains therefore often mandatory ${ }^{14,15,16,17}$.

Prior to our work with nanosilver coatings, we compared two commercially available hemocompatible coatings (BioLine- and X-coating) and 
a standard uncoated tubing material in an in vitro model that mimics blood flow over an artificial surface for several hours. Fresh whole blood was circulated over the material surfaces for a period of $5 \mathrm{~h}$. The experimental results had two important implications for the development of new surface coatings, which are not only antithrombotic but also anti-infective. First, it was observed that bloodmaterial contact in the Chandler loop resulted in a slight, but gradual increase of multiple parameters (free hemoglobin (free $\mathrm{Hb}$ ), terminal complement complex (TCC), myeloperoxidase (MPO), $\beta$-thromboglobulin ( $\beta$-TG)). This was observed in all three materials investigated. Additionally, the scanning electron microscopy (SEM) analysis revealed that none of the materials evaluated were free from interactions with blood components. Although the extent to which parameters of hemolysis, complement, and leukocyte and platelet activation increased could be clinically non-significant, it indicates that even state-of-the art materials - available for prevention of thrombosis - do not completely leave the blood homeostasis mechanisms unaffected. This highlights the importance to investigate the blood compatibility of all materials used in blood-contacting materials, certainly for new materials of which knowledge about blood-material interactions is lacking, such as nanosilver. Secondly, differences between the investigated hemocompatible coatings, BioLine and X-coating, became apparent only after long term circulation of fresh whole blood over the material surfaces. Differences between coatings thus might masquerade during the first hours of blood contact. The Chandler loop seems a challenging test method for coating materials, which can be used to expose materials to flowing blood for relatively long periods of time.

One could speculate on the possibility to use the Chandler loop for even longer duration than $5 \mathrm{~h}$. It must be kept in mind, however, that blood is circulated in this model without oxygenation. At a given time point the dying of blood cells will cause artefacts. A recent large study by Jackman et al. investigated the effect of blood sample age at the time of centrifugation and analysis of plasma cytokine concentrations in healthy donors ${ }^{46}$. A total number of 41 soluble immune mediators were examined, among which 16 mediators, including MPO, showed unstable concentrations over a 4-day period. MPO was also used as a leukocyte activation marker in Chapter 2. Whether the increases in MPO levels observed in our Chandler loop model were artefactual is unlikely. Jackman and colleagues reported a mean increase in MPO levels of $28 \%$ per day from their experiments ${ }^{46}$. We observed a fourfold increase in MPO levels within $5 \mathrm{~h}$ in our Chandler loop model. This much steeper increase in MPO concentrations over a much shorter time span is believed to be due largely to blood-material contact within the tubing loops. 


\section{Blood compatibility of coatings containing silver nanoparticles}

Although material-induced thrombosis and infection are interrelated, most research to design surface coatings that are able to prevent CRBSIs focused solely on coatings embedded with antibiotics or antiseptics ${ }^{10,1,12,13}$. In particular AgNPs have gained much attention during the last years, as a candidate material for development of new antimicrobial catheter coatings $31,32,3,3,34,35,36,37,38$. Knowledge about the blood compatibility of AgNPs was, however, completely lacking. Hence this could be a relevant factor in the design of safe and effective antimicrobial coatings for blood-contacting devices.

The work presented in Chapters 3-5 shows some of the first data about the hemocompatibility of coatings embedded with AgNPs. In Chapter 3, contact between platelet-rich plasma (PRP) and a set of coatings with increasing AgNP-concentrations resulted in decreased thrombin generation lag times (TGTlag) and this effect seemed to be dose-dependent. Furthermore, platelet activation was increased greatly compared to coatings without AgNPs. In Chapter 4, however, contact between PRP and silver-bromide (AgBr) nanocomposite-coatings resulted in only marginal effects on TGTlag, while platelet activation was again clearly elevated. Additionally, SEM analysis of platelets adhered to AgBr-nanocomposite coatings showed distorted morphologies. Interestingly, the effects observed throughout these chapters were different between coatings embedded with nanosilver from different sources.

Upon oxidation, AgNPs release $\mathrm{Ag}^{+}$in the surrounding medium and acquire an outer surface covered with chemisorbed $\mathrm{Ag}^{+}{ }^{34}$. Prompted to know if one of these mechanisms had a dominant role in the observed effects, a set of titration experiments was performed (Chapter 3). The results indicated that the observed thrombogenic and platelet activating effects may be due to a mechanism working at the interface between the coating and the PRP, instead of released $\mathrm{Ag}^{+}$. This might imply contact between the AgNPs positioned at the surface of the coating and blood proteins or cells, with transfer of chemisorbed $\mathrm{Ag}^{+}$from oxidized AgNPs to cells or proteins ${ }^{47}$. On the other hand, with respect to the observed antimicrobial effects, data from our titration experiments did not rule out elution of $\mathrm{Ag}^{+}$or direct nanoparticle-contact as a mechanism of action. This latter topic was recently studied by Sotiriou, et al. and Sintubin, et al. ${ }^{48,49}$. The work from these groups points towards $\mathrm{Ag}^{+}$-release as the dominant mechanism in the antibacterial activity of AgNPs. The authors, however, do not exclude direct contact between AgNPs and bacteria as a secondary antibacterial mechanism. The effects of direct contact with AgNPs are much less explored for blood proteins or platelets. Further insights in the exact mechanisms by which AgNPs affect the coagulation cascade or platelets can, however, provide key points for modification of AgNP-effects. 
Recently, other research groups published about the interactions between AgNPs and blood. Unlike our studies, where blood components were exposed to AgNPs embedded in a coating matrix, these studies investigated direct exposure of blood to AgNPs. Shrivastava, et al. exposed washed platelets to AgNPs and found that the AgNPs had an inhibiting effect on the integrinmediated platelet functional responses aggregation, secretion, adhesion to immobilized fibrinogen or collagen and retraction of fibrin clot ${ }^{50}$. The AgNPs had a perturbing effect on the platelet membrane microenvironment and accumulated in granules and vacuolar spaces. The authors ascribed their observed effects to direct interactions of AgNPs with the platelet machinery. Within the same study, a series of in vivo experiments was also performed, using a mouse model with intravenous injection of AgNP-suspension. Blood collected from AgNP-exposed animals also indicated platelet inhibiting effects of AgNPs, as measured by decreased platelet aggregation ${ }^{50}$. The same authors published another study in which they investigated interactions between AgNPs and fibrinogen ${ }^{51}$. From their observations it appears that AgNPs can interact with fibrinogen, resulting in conformational changes in protein structure and retardation of fibrinogen polymerization kinetics ${ }^{51}$. Results from this research group indicate that a direct contact mechanism between AgNPs and blood components is feasible and can disturb normal blood homeostasis. Jun and colleagues incubated washed platelets in vitro with AgNPs and observed increased platelet aggregation and procoagulant activity ${ }^{52}$. In the same study, in vivo experiments comprising a rat model with intravenous injection of AgNP suspension, followed by induced thrombosis, showed increased thrombus weight in AgNP-exposed rats ${ }^{52}$. Finally, Hulander and colleagues investigated contact of whole blood with noble metal coated surfaces instead of nanoparticles ${ }^{53}$. After $1 \mathrm{~h}$ exposure of whole blood to noble metal-coated surfaces, complement activation and fibrinogen adsorption was highest on silver-coated surfaces, compared to other materials tested. Platelet depletion was, however, low on silver-coated surfaces, as was activation of the coagulation cascade, indicated by only low generation of thrombin-antithrombin complexes ${ }^{53}$. In line with our results on the blood compatibility of nanosilver coatings, in particular with respect to thrombin generation data, the results presented by these other research groups are also contradictory.

Probably, factors relating to the synthesis method of the nanoparticles are responsible for the currently found contradictory results of AgNP blood compatibility. A review of the literature reveals that, dependent on their method of synthesis, AgNPs can differ in terms of shape, size and phase ${ }^{54}$, or can be modified resulting in different surface charge ${ }^{55}$. Spinning disc processing technology represents a new technology that allows precise control over these mentioned factors during nanoparticle synthesis ${ }^{54}$. Toxicity studies have reported that such factors, like size ${ }^{56}$, surface charge ${ }^{55}$ and surface chemistry ${ }^{57,58}$ 
of AgNPs, strongly determine their toxic effects. Likely, these factors also play a role in the interaction between AgNPs and contacting blood components. No studies, however, have addressed this question yet.

\section{The blood compatibility of dual action silver nanoparticle-heparin coatings}

The information from Chapters 2-4 supported the rationale to design combinatory coatings, embedded with both antimicrobial AgNPs and a drug to prevent possible prothrombotic effects of AgNPs. In Chapter 5 we succeeded in making coatings embedded with both heparin and AgNPs, and a set of control coatings with either heparin alone, AgNPs alone, or neither heparin and AgNPs. XPS confirmed the presence of heparin and AgNPs where added.

A first series of experiments with these coatings gave some remarkable results. It was shown that the presence of heparin together with AgNPs did not result in lesser antimicrobial activity of the AgNPs. In contrary, the coating matrix loaded with only heparin even showed an antimicrobial effect of the heparin itself - in terms of lesser bacterial adhesion - when compared with the coating matrix without both heparin and AgNPs. These data are in line with clinical data from a retrospective study by Jain, et al. ${ }^{59}$. They investigated the use of heparin-coated versus standard uncoated tunnelled dialysis catheters in 175 hemodialysis patients. Primary end-points were the frequency of CRBSIs and cumulative patency of the catheters. The authors found no differences between both catheter types in terms of catheter patency. However, the frequency of CRBSIs was reduced in heparin coated catheters ${ }^{59}$. Although this hypothesis has to be further examined by randomized clinical trials, the data of Jain, et al. indicate the importance of addressing infection and thrombosis in a combined manner.

An antimicrobial effect was found for all nanosilver coatings tested throughout this thesis. This is in line with the general finding in literature that nanosilver exhibits strong antimicrobial properties and it underlines its potential for use in antimicrobial catheter coatings. On the other hand, the presence of nanosilver together with heparin did not negatively affect the action of heparin. All coatings loaded with heparin and nanosilver did not show thrombus formation for the total duration of the thrombin generation experiment $(1 \mathrm{~h})$.

An interesting finding was that the thrombin generation experiments could not find differences between the Ag6V- and Ag4E nanoparticle coatings (Chapter 5) and the control coatings without AgNPs. This is in line with the thrombin generation experiments with AgBr-nanocomposite coatings, presented in Chapter 4, and their control materials without nanosilver. As discussed earlier, specific physicochemical properties of AgNPs, like shape, charge, size or 
coating, ${ }^{54,55,56,57,58}$ may have caused the discordance between prothrombotic effects of nanosilver coatings found in Chapter 3 and the absence of these effects found in Chapters 4 and 5. The fact that no clear prothrombotic effect was measured for coatings with Ag6V- or Ag4E-nanosilver indicates that these AgNPs may be suited for the development of coatings that are both antimicrobial, but not thrombogenic.

\section{Conclusions and future perspectives}

Throughout this thesis, we investigated the blood compatibility of coating materials relevant for both the clinical practice of CPB/ELS and (central venous) catheterization. The information presented here is relevant for the development of new coating materials that address both material-induced thrombosis and infection. Important aspects for future research are that coatings with AgNPs from different sources can behave differently with respect to blood compatibility. On one end of the spectrum, it appears that coatings embedded with AgNPs can elicit prothrombotic and platelet activating responses. When developing antimicrobial nanosilver coatings for blood contacting devices, attention should therefore be paid to carefully choosing AgNPs with welldocumented blood compatibility. Another important issue is that the concept of dual action coatings seems plausible for the design of antimicrobial nanosilver coatings with safe blood compatibility. The combined presence of an antithrombotic or antiplatelet drug might ensure better safety of coating materials with AgNPs, which currently have only limited documentation on their blood compatibility. Since nanosilver is becoming a popular material to create medical antimicrobial surfaces, this is highly relevant with respect to product safety.

A starting point for future research could be to investigate the abovementioned AgNP-characteristics like shape, size and surface characteristics, with specific focus on a possible role during blood contact. Studies on the blood compatibility of nanosilver should involve direct contact between blood components and AgNPs and analysis of coagulatory and platelet function, as well as complement activation in relation to their physical and chemical characteristics. Ideally, future research would identify a set of AgNPcharacteristics that allow blood contact without interference with the coagulation cascade and/or blood platelets, but with preserved antimicrobial properties.

Aside from studying direct interactions between blood and AgNPs, it is also worthwhile to compare AgNPs with different physicochemical properties when embedded in a blood-contacting coating. By no means can it be accepted that AgNPs embedded in a surrounding coating matrix will display the same blood compatibility as during direct contact with the blood. Protein adhesion, 
Chapter 6

for example, which is an initial step during blood-biomaterial contact, could be different on "naked" AgNPs than on the surface of a coated AgNP. 


\section{References}

1 Issitt, R., Cumberland, T., Clements, A. \& Mulholland, J. Clinical evaluation of the Admiral $1.35 \mathrm{~m} 2$ hollow-fibre membrane oxygenator. Perfusion 23, 33-38 (2008).

2 Cesar, S., Garcia, A., Parada, E. \& Soriano, A. Cavernous sinus thrombosis due to invasive community-associated methicillin-resistant Staphylococcus aureus infection. Enferm Infecc Microbiol Clin 28, 755-756, (2010).

3 Keller, J. E., Hindman, J. W., Mehall, J. R. \& Smith, S. D. Enoxaparin inhibits fibrin sheath formation and decreases central venous catheter colonization following bacteremic challenge. Crit Care Med 34, 1450-1455, (2006).

4 Mehall, J. R., Saltzman, D. A., Jackson, R. J. \& Smith, S. D. Catheter materials affect the incidence of late blood-borne catheter infection. Surg Infect (Larchmt) 2, 225-229; discussion 229-230, (2001).

5 Mehall, J. R., Saltzman, D. A., Jackson, R. J. \& Smith, S. D. Fibrin sheath enhances central venous catheter infection. Crit Care Med 30, 908-912 (2002).

6 Lawson, D. S. et al. North American neonatal extracorporeal membrane oxygenation (ECMO) devices and team roles: 2008 survey results of Extracorporeal Life Support Organization (ELSO) centers. J Extra Corpor Technol 40, 166-174 (2008).

7 Sievert, A. N., Shackelford, A. G. \& McCall, M. M. Trends and emerging technologies in extracorporeal life support: results of the 2006 ECLS survey. J Extra Corpor Technol 41, 73-78 (2009).

8 Tagarakis, G. I. \& Tsilimingas, N. B. Heparin-coated extracorporeal circulation systems in heart surgery. Recent Pat Cardiovasc Drug Discov 4, 177-179, (2009).

9 Wendel, H. P. \& Ziemer, G. Coating-techniques to improve the hemocompatibility of artificial devices used for extracorporeal circulation. Eur J Cardiothorac Surg 16, 342-350, (1999).

10 Cicalini, S., Palmieri, F. \& Petrosillo, N. Clinical review: new technologies for prevention of intravascular catheter-related infections. Crit Care 8, 157-162, (2004).

11 Frasca, D., Dahyot-Fizelier, C. \& Mimoz, O. Prevention of central venous catheter-related infection in the intensive care unit. Crit Care 14, 212, (2010).

12 Pai, M. P., Pendland, S. L. \& Danziger, L. H. Antimicrobial-coated/bonded and -impregnated intravascular catheters. Ann Pharmacother 35, 1255-1263 (2001).

13 Ramritu, P. et al. A systematic review comparing the relative effectiveness of antimicrobialcoated catheters in intensive care units. Am J Infect Control 36, 104-117, (2008).

14 Fromes, Y. et al. A comparison of low vs conventional-dose heparin for minimal cardiopulmonary bypass in coronary artery bypass grafting surgery. Anaesthesia 66, 488-492, (2011).

15 Gunaydin, S. et al. Clinical and biomaterial evaluation of hyaluronan-based heparin-bonded extracorporeal circuits with reduced versus full systemic anticoagulation in reoperation for coronary revascularization. J Cardiovasc Med (Hagerstown) 10, 135-142, (2009).

16 Ovrum, E. et al. Heparinized cardiopulmonary bypass circuits and low systemic anticoagulation: An analysis of nearly 6000 patients undergoing coronary artery bypass grafting. J Thorac Cardiovasc Surg 141, 1145-1149, (2011).

17 Ranucci, M., Balduini, A., Ditta, A., Boncilli, A. \& Brozzi, S. A systematic review of biocompatible cardiopulmonary bypass circuits and clinical outcome. Ann Thorac Surg 87, 1311-1319, (2009).

18 Halton, K. \& Graves, N. Economic evaluation and catheter-related bloodstream infections. Emerg Infect Dis 13, 815-823 (2007).

19 Halton, K. A., Cook, D. A., Whitby, M., Paterson, D. L. \& Graves, N. Cost effectiveness of antimicrobial catheters in the intensive care unit: addressing uncertainty in the decision. Crit Care 13, R35, (2009). 
20 Hockenhull, J. C. et al. The clinical effectiveness and cost-effectiveness of central venous catheters treated with anti-infective agents in preventing bloodstream infections: a systematic review and economic evaluation. Health Technol Assess 12, iii-iv, xi-xii, 1-154, (2008).

21 Barrett, C. S. et al. Neurological injury after extracorporeal membrane oxygenation use to aid pediatric cardiopulmonary resuscitation. Pediatr Crit Care Med 10, 445-451, (2009).

22 Fischer, S. et al. Extracorporeal membrane oxygenation for primary graft dysfunction after lung transplantation: analysis of the Extracorporeal Life Support Organization (ELSO) registry. J Heart Lung Transplant 26, 472-477, (2007).

23 Gow, K. W. et al. Extracorporeal life support for support of children with malignancy and respiratory or cardiac failure: The extracorporeal life support experience. Crit Care Med 37, 1308-1316, (2009).

24 Ko, W. J., Hsu, H. H. \& Tsai, P. R. Prolonged extracorporeal membrane oxygenation support for acute respiratory distress syndrome. J Formos Med Assoc 105, 422-426, (2006).

25 Linden, V. et al. High survival in adult patients with acute respiratory distress syndrome treated by extracorporeal membrane oxygenation, minimal sedation, and pressure supported ventilation. Intensive Care Med 26, 1630-1637 (2000).

26 Megarbane, B. et al. Emergency feasibility in medical intensive care unit of extracorporeal life support for refractory cardiac arrest. Intensive Care Med 33, 758-764, (2007).

27 Meister, B. et al. Extracorporeal membrane oxygenation as a rescue therapy for leukaemic children with pulmonary failure. BrJ Haematol 148, 126-131, (2010).

28 Mongero, L. B. et al. Extracorporeal membrane oxygenation for diffuse alveolar hemorrhage and severe hypoxemic respiratory failure from silicone embolism. Perfusion 25, 249-252; discussion 253-244, (2010).

29 Blot, S. I. et al. Clinical and economic outcomes in critically ill patients with nosocomial catheter-related bloodstream infections. Clin Infect Dis 41, 1591-1598, (2005).

30 O'Grady N, P. et al. Guidelines for the prevention of intravascular catheter-related infections. Am J Infect Control 30, 476-489, (2002).

$31 \mathrm{He}, \mathrm{T}$. \& Chan, V. Covalent layer-by-layer assembly of polyethyleneimine multilayer for antibacterial applications. J Biomed Mater Res A 95, 454-464, (2010).

32 Hsu, S.-h., Tseng, H.-J. \& Lin, Y.-C. The biocompatibility and antibacterial properties of waterborne polyurethane-silver nanocomposites. Biomaterials 31, 6796-6808 (2010).

33 Hung, H.-S. \& Hsu, S.-H. Biological performances of poly(ether)urethane-silver nanocomposites. Nanotechnology 18, 475101 (2007).

34 Lok, C. N. et al. Silver nanoparticles: partial oxidation and antibacterial activities. J Biol Inorg Chem 12, 527-534, (2007).

35 Maki, D. G. In vitro studies of a novel antimicrobial luer-activated needleless connector for prevention of catheter-related bloodstream infection. Clin Infect Dis 50, 1580-1587, (2010).

36 Roe, D., Karandikar, B., Bonn-Savage, N., Gibbins, B. \& Roullet, J. B. Antimicrobial surface functionalization of plastic catheters by silver nanoparticles. J Antimicrob Chemother 61, 869-876, (2008).

37 Tseng, K. H. \& Liao, C. Y. Production of silver ions from colloidal silver by nanoparticle iontophoresis system. J Nanosci Nanotechnol 11, 1991-1995 (2011).

38 Yao, Y., Ohko, Y., Sekiguchi, Y., Fujishima, A. \& Kubota, Y. Self-sterilization using silicone catheters coated with $\mathrm{Ag}$ and TiO2 nanocomposite thin film. J Biomed Mater Res B Appl Biomater 85, 453-460, (2008).

39 Baker, C., Pradhan, A., Pakstis, L., Pochan, D. J. \& Shah, S. I. Synthesis and antibacterial properties of silver nanoparticles. J Nanosci Nanotechnol 5, 244-249 (2005).

40 Gunaydin, S., McCusker, K. \& Vijay, V. Clinical and biomaterial evaluation of a new condensed dual-function extracorporeal circuit in reoperation for coronary artery bypass surgery. Int J Artif Organs 32, 802-810, (2009). 
41 Gunaydin, S. et al. Hyaluronan based heparin free coated open and closed extracorporeal circuits for high risk coronary revascularization. J Extra Corpor Technol 42, 286-292 (2010).

42 Marcoux, J. et al. Outcomes comparison of 5 coated cardiopulmonary bypass circuits versus an uncoated control group of patients undergoing cardiac surgery. Perfusion 24, 307-315, (2009).

43 Sohn, N., Marcoux, J., Mycyk, T., Krahn, J. \& Meng, Q. The impact of different biocompatible coated cardiopulmonary bypass circuits on inflammatory response and oxidative stress. Perfusion 24, 231-237, (2009).

44 Melvin, M. E., Fissell, W. H., Roy, S. \& Brown, D. L. Silicon induces minimal thromboinflammatory response during 28-day intravascular implant testing. ASAIO J 56, 344-348, (2010).

45 Yu, K. et al. Clinical Evaluation of Two Different Extracorporeal Membrane Oxygenation Systems: A Single Center Report. Artif Organs, (2011).

46 Jackman, R. P. et al. Effects of blood sample age at time of separation on measured cytokine concentrations in human plasma. Clin Vaccine Immunol 18, 318-326, (2011).

47 Stevens, K. N. et al. The relationship between the antimicrobial effect of catheter coatings containing silver nanoparticles and the coagulation of contacting blood. Biomaterials 30, 3682-3690, (2009).

48 Sintubin, L. et al. The antibacterial activity of biogenic silver and its mode of action. Appl Microbiol Biotechnol 91, 153-162, (2011).

49 Sotiriou, G. A. \& Pratsinis, S. E. Antibacterial activity of nanosilver ions and particles. Environ Sci Technol 44, 5649-5654, (2010).

50 Shrivastava, S. et al. Characterization of antiplatelet properties of silver nanoparticles. ACS Nano 3, 1357-1364, (2009).

51 Shrivastava, S. et al. Negative regulation of fibrin polymerization and clot formation by nanoparticles of silver. Colloids Surf B Biointerfaces 82, 241-246, (2011).

52 Jun, E. A. et al. Silver nanoparticles enhance thrombus formation through increased platelet aggregation and procoagulant activity. Nanotoxicology 5, 157-167, (2011).

53 Hulander, M. et al. Blood interactions with noble metals: coagulation and immune complement activation. ACS Appl Mater Interfaces 1, 1053-1062, (2009).

54 Iyer, K. S., Raston, C. L. \& Saunders, M. Continuous flow nano-technology: manipulating the size, shape, agglomeration, defects and phases of silver nano-particles. Lab Chip 7, 1800-1805, (2007).

55 El Badawy, A. M. et al. Surface charge-dependent toxicity of silver nanoparticles. Environ Sci Technol 45, 283-287, (2011).

56 Carlson, C. et al. Unique cellular interaction of silver nanoparticles: size-dependent generation of reactive oxygen species. J Phys Chem B 112, 13608-13619, (2008).

57 Ahamed, M. et al. DNA damage response to different surface chemistry of silver nanoparticles in mammalian cells. Toxicol Appl Pharmacol 233, 404-410, (2008).

58 Schrand, A. M., Braydich-Stolle, L. K., Schlager, J. J., L., D. \& Hussain, S. M. Can silver nanoparticles be useful as potential biological labels? Nanotechnology 19, 235104 (2008).

59 Jain, G., Allon, M., Saddekni, S., Barker, J. F. \& Maya, I. D. Does heparin coating improve patency or reduce infection of tunneled dialysis catheters? Clin J Am Soc Nephrol 4, 1787-1790, (2009). 
